The SCIENCE-Policy INTERfaCe IN Transboundary Water MaNagement Regimes:

The Case of Lake Victoria

by

Christina S. Cheng, B.Sc. (Hons)

\author{
A thesis presented to \\ Ryerson University \\ in partial fulfillment of the \\ requirements for the degree of \\ Master of Applied Science \\ in the Program of \\ Environmental Applied Science and Management
}

Toronto, Ontario, Canada, 2010

${ }^{\circ}$ Christina Cheng, 2010 
I hereby declare that I am the sole author of this thesis.

I authorize Ryerson University to lend this thesis to other institutions or individuals for the purpose of scholarly research.

I further authorize Ryerson University to reproduce this thesis by photocopying or by other means, in total or in part, at the request of other institutions or individuals for the purpose of scholarly research. 


\section{The SCIENCE-POLICY INTERFACE IN \\ Transboundary Water Management Regimes: \\ The Case OF Lake Victoria}

Master of Applied Science 2010

Christina S. Cheng

Program of Environmental Applied Science and Management

Ryerson University

\section{Abstract}

The degree that science is integrated into environmental policy processes is dependent on the policymakers' perception of the role and utilization of science in policy development. Using existing literature and interviews with key individuals in North America and Uganda engaged in environmental policy development, this thesis argues that adopting a positivist approach to policy development strengthens the science-policy interface and can result in more effective policies. This interface is examined in the context of transboundary water management, and specifically in East Africa's Lake Victoria management regime. This paper illustrates how the relationship between science and policy is evolving in a difficult ecological, socio-economic and political setting, and how the tensions that exist are attempting to be resolved. It is observed that the science-policy divergence can be exacerbated in transboundary areas. This requires scientists and policymakers to be cognizant of these challenges, and to adopt tools to strengthen the interface for the development of effective transboundary water management regimes. 


\section{ACKNOWLEDGEMENTS}

It is with great sincerity that I express gratitude to all the individuals who agreed to be interviewed for this study. I thank you for your time, openness and willingness to participate in this thesis project. Your knowledge, professionalism and dedication to environmental protection are invaluable, inspirational and promising. In particular, I acknowledge in no particular order staff at the International Joint Commission, Great Lakes Fisheries Commission, Ontario Ministry of the Environment, Environment Canada, United Nations University-Institute for Water, Environment and Health, Nile Basin Initiative, Uganda Ministry of Water and EnvironmentDirectorate of Water Resources Management, National Environment Management Authority, Lake Victoria Fisheries Organization, National Fisheries Resources Research Institute, Makerere University-Institute of Environment and Natural Resources, Gaba Beach Management Unit, Global Water Partnership and the Global Environment Facility. I also thank Ken Ogilvie and Boaz Kiezire who shared invaluable knowledge gained in their previous roles with policy development and water management.

I would like to specifically acknowledge Godber Tumushabe of Advocates Coalition for Development and Environment. Thank you for graciously opening your doors and agreeing to be my academic affiliation during my stay in Kampala.

Thank you to my Ugandan host family, the Walusimbi's, who made my stay in Kampala that much more enjoyable.

I would like to express gratitude to those who have guided me throughout this journey. First, I would like to thank Ed Piché, without whom I would not have endeavoured to undertake this challenge at the time that I did. Thank you to Dan Allen and John McDonald, who have supported me not only in this effort but who have been godsends from the very beginning of my professional career. I would also like to thank the Ontario Ministry of the Environment for supporting my desire for higher education and specifically to Daniel Cayen, Gary De Brou, Jim Whitestone, Heather Pearson, Karen Clark and Adam Redish.

Thank you to Christopher Gore, my academic advisor, for your belief and faith in the work that we do. And of course, for your utmost patience, guidance, support, humour and humility.

Lastly, a sincere thank you to my wonderful family and friends...words cannot even begin to express the gratitude I have for you. 


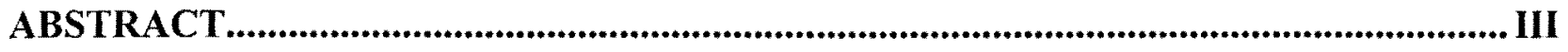

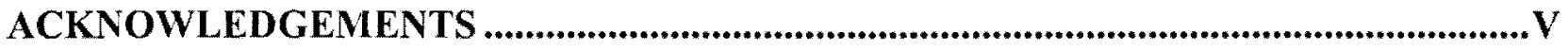

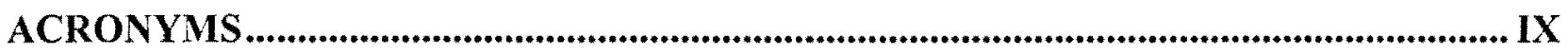

CHAPTER 1: INTRODUCTION

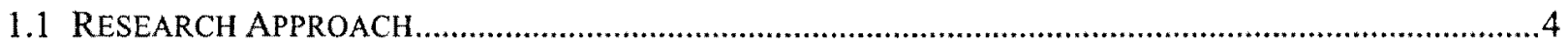

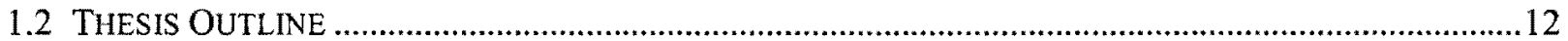

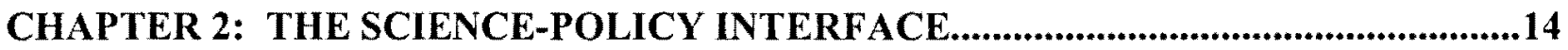

2.1 SCIENCE IN THE ENVIRONMENTAL POLICY DEVELOPMENT PROCESS .............................................15

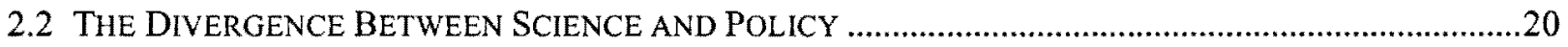

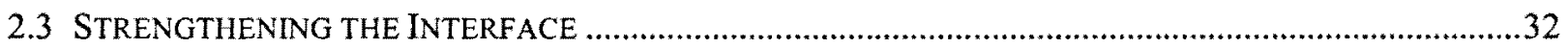

2.4 SCIENCE AND POLICY IN TRANSBOUNDARY WATER MANAGEMENT: AN INTRODUCTION ...............41

CHAPTER 3：TRANSBOUNDARY WATER MANAGEMENT .........................................44

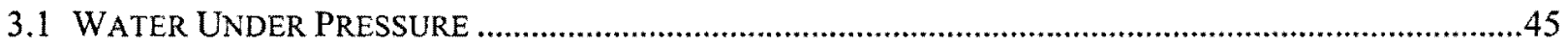

3.2 APPROACHES TO MANAGING TRANSBOUNDARY WATERS ………................................................4

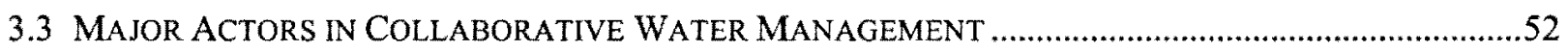

3.4 CRITERIA For EFFECTIVE TRANSBOUNDARY WATER MANAGEMENT ……………….....................58

CHAPTER 4: THE SCIENCE-POLICY CONNECTION IN TRANSBOUNDARY

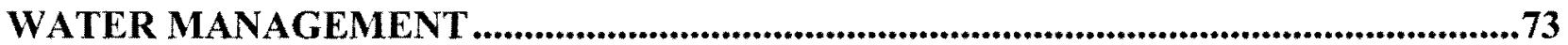

4.1 THE SCIENCE-POLICY DisConNECT IN TRANSBoundary WATER MANAGEMENT...........................74

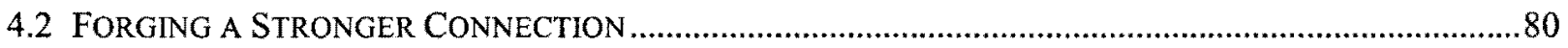

4.3 APPROACH TO MANAGING THE NORTH AMERICAN GREAT LAKES.............................................8

CHAPTER 5: LAKE VICTORIA: A CASE STUDY ON AN EMERGING TRANSBOUNDARY WATER MANAGEMENT REGIME .................................................99

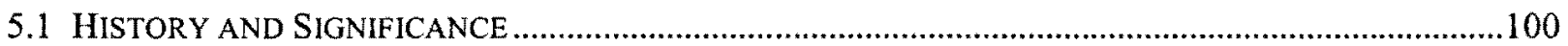

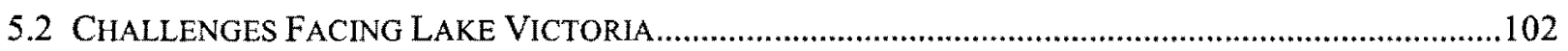

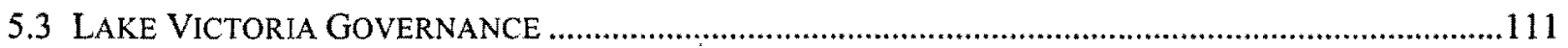

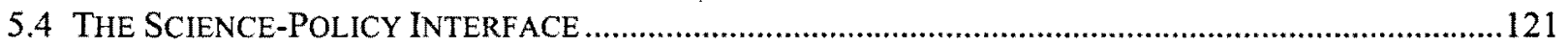

5.5 INTEGRATING SCIENCE INTO THE LAKE VICTORIA MANAGEMENT REGIME ...................................1.124

5.6 CHALLENGES OF NARROWING THE SCIENCE-POLICY GAP............................................................134

5.7 EVIDENCE OF SCIENTIFIC INFLUENCE ON POLICY AND PROJECT OUTCOMES ....................................139

5.8 THE FUTURE OF LAKE VICTORIA MANAGEMENT ……..............................................................145

CHAPTER 6: CONCLUSIONS AND RECOMMENDATIONS.......................................147

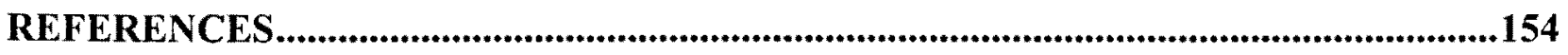

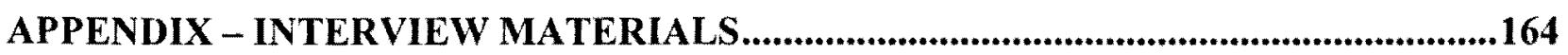


List OF Figures AND TABLES

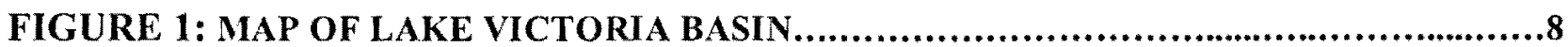

FIGURE 2: INTEGRATING SCIENCE INTO THE ENVIRONMENTAL POLICY

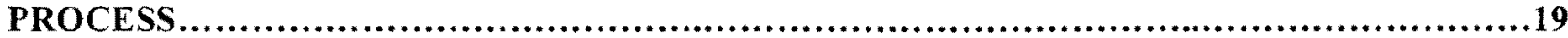

FIGURE 3: PRINCIPAL EVENTS IN THE RECENT ENVIRONMENTAL HISTORY OF LAKE VICTORIA, IN RELATION TO HUMAN-POPULATION GROWTH AND

AGRICULTURAL PRODUCTION IN ITS DRAINAGE BASIN........................................99

TABLE 1: CHARACTERISTICS OF SCIENCE AND GOVERNMENT

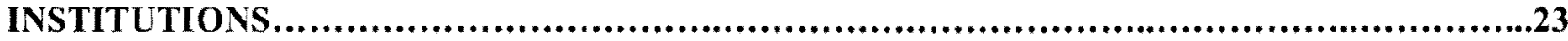

TABLE 2: FUNCTIONS OF SCIENTIFIC INFORMATION MODEL (FOSI) LINKING

SCIENCE AND POLICY...........................................................................................................................34 
ACRONYMS

ACODE Advocates Coalition for Development and Environment

CCME Canadian Council of Ministers of the Environment

CIFA Committee for Inland Fisheries of Africa

CIFAA Committee for Inland Fisheries and Aquaculture of Africa

CNWRI Canadian National Water Research Institute

COA Canada-Ontario Agreement

CRU Climate Research Unit

CSO Civil Society Organization

CSTA Council of Science and Technology Advisors

DWRM Directorate of Water Resources Management

EAC East African Community

EAFRO East African Fisheries Research Organization

EAFFRO East African Freshwater Fisheries Research Organization

ENGO Environmental Non-Government Organization

FAO Food and Agriculture Organization

FOSI Functions of Scientific Information Model

GEF Global Environment Facility

GIS Geographic Information System

GLFC Great Lakes Fisheries Commission

GLWQA Great Lakes Water Quality Agreement

GWP Global Water Partnership

IEG Independent Evaluation Group

IJC International Joint Commission

IUCN International Union of Conservation of Nature

IWRM Integrated Water Resources Management

LVBC Lake Victoria Basin Commission

LVEMP Lake Victoria Environmental Management Project

LVFO Lake Victoria Fisheries Organization

LVFRP Lake Victoria Fisheries Research Project

NaFIRRI National Fisheries Resources Research Institute

NBI Nile Basin Initiative

NEMA National Environment Management Authority

NIS Nile Information System

PARI Public Agricultural Research Institute

$\mathrm{SAB} \quad$ Science Advisory Board

SOLEC State of the Lake Ecosystem Conference

UEA University of East Anglia

UN United Nations

USEPA United States Environmental Protection Agency

WQB Water Quality Board 


\section{Chapter 1: Introduction}

There is a perception that policy processes follow linear paths (Keller, 2009, p. 6). From the identification of an issue to the implementation of a policy, a logical progression of steps can be generally articulated for how policy evolves. In reality, policy processes are much more convoluted and can diverge along many paths prior to reaching the intended destination (Keller, 2009, p. 6). This is particularly evident in the environmental policy realm where ecological, socio-economic and political factors can influence policy direction and outcomes. As a result of the complexity of environmental issues, policy objectives can only be defined and attained when the confluence of these factors reaches a point where governments decide that a sufficient balance has been reached (Keller, 2009; Kingdon, 1995). There are a number of factors that can inform and influence how policymakers determine this 'point of optimization'. One significant factor in environmental policy development is scientific knowledge.

There are various points in the policy process where science can be integrated. In this thesis this is described as the science-policy interface. The point at which science is engaged is determined by how it is perceived and subsequently utilized by policymakers. In situations where policy objectives are predetermined, science is characterized as a 'means to an end' and is only engaged when a solution is required to meet the intended goal. In other scenarios, science is engaged when defining the policy objective and is used to inform policy direction and can shape policy outcomes. These different perspectives can influence the degree that science is integrated into the policy process. Circumscribing scientific integration can weaken the interface and create a divergence between science and policy. There are various reasons that can contribute to the widening of this gap including a lack of understanding of objectives and processes between the disciplines, to deeper engrained barriers such as paradigms and 
institutional structures. While there is an abundance of literature on how science and policy diverge, which concludes that there is a need to cultivate stronger relationships between the two communities, surprisingly little research is available that identifies how this can be done or is done (Schaefer \& Bielak, 2006). Given that scientific evidence has the potential to guide environmental policy development and influence outcomes, there is high interest in identifying tools and mechanisms that can strengthen the science-policy connection.

This thesis examines the relationship between science and policy in the management of transboundary water management regimes. Transboundary management regimes are an important environmental domain to study the science-policy relationship given their complexity and the heightened convergence of social, economic, and ecological issues they illuminate. This paper uses the case of East Africa's Lake Victoria to illustrate how the relationship between science and policy is evolving and how the tensions that exist between the two are attempting to be resolved. The analysis is informed by informal comparison to other transboundary water management regimes and through data collected in interviews with key informants in North America and Uganda.

Transboundary or international water bodies are receiving greater attention by the international community. They are becoming areas of increased conflict as a result of competing demands, water scarcity and consequent security. It is anticipated that transboundary areas will be increasingly developed in response to growing populations and economies and could potentially aggravate political tensions (Varis, Tortajada \& Biswas, 2008, p. 3; GEF, 2009). Effectively managing these shared resources is critical for the sustainability of the resource and to prevent conflict between riparian countries. Despite this, the magnitude of issues and number 
of actors involved with transboundary water management can exacerbate the divergence between science and policy.

This thesis argues that strengthening the science-policy interface in environmental policy processes enhances the credibility of policies and the process itself, and further, is critical for the development of effective policies. Effective policies result in improvements to the environment that can be measured or empirically observed. These policies address sustainable management of natural resources while balancing the socio-economic and political demands of a society. It is both responsible and prudent for policymakers and scientists to undertake efforts to narrow the divide between the two communities to ensure policy decisions and outcomes are informed by the latest and most sound scientific information.

More specifically, I argue that the causes for the science-policy gap go beyond the disciplinary differences commonly observed in environmental policy processes generally. The divergence in transboundary areas can be exacerbated due to variations in national approaches, cultures and demands on the resource. In particular, some of the causes identified for this disconnect include policy and research fragmentation, political 'readiness' and politicization of science. Strengthening the science-policy interface has the potential to alleviate these challenges and cultivate stronger relations among the riparian countries, which are necessary for the development of holistic and integrated water management practices. This paper also demonstrates how policy venues and the maturity of the process can influence the ebbs and flows of scientific integration. Building on a detailed review of the literature and interview data, the thesis reveals a number of tools and mechanisms that are needed and can be used to improve the interface between science and policy, such as enhancing communication and engaging 
stakeholders in the policy process. All of these efforts can help forge stronger relations between the science and policy realms.

Lake Victoria is East Africa's Great Lake, and the second largest freshwater lake in the world by surface area. It borders some of the world's poorest nations and is the lifeline for countries in the Nile basin (Klohn \& Andjelic, 2008). Its resources support approximately 30 million people in the basin and population projections estimate this will double by the year 2020 (Awange \& Ong'ang'a, 2006; Kayombo \& Jorgensen, 2006). Growing populations and economies in and around the lake are intensifying the pressures on its resources including decreasing fish stocks and deteriorating water quality. Lake Victoria is emerging as a region of rapid ecological deterioration as a result of these increasing demands, risking permanent changes in ecosystem function. Collaborative management regimes are nascent and have been evolving with the assistance of international partners over the past two decades. Science-policy linkages are being explored and have not been fully defined (United Nations University, 2008). This provides an opportunity to examine the role of science in a management regime that is emerging.

Forging stronger connections between the science and policy realms has the potential to result in more effective policies for environmental protection. There are, however, challenges that perpetuate the incessant divergence between these two communities. Exploring the causes of this disconnect and identifying instruments that could bridge this gap is a means to strengthen the science-policy connection. This is particularly important in transboundary water areas where the complexities of management can further divide these communities.

\subsection{Research Approach}

In conducting this study, a literature review was conducted on the topics of the sciencepolicy interface and transboundary water management to establish the knowledge base required 
for the second phase of the study, which entailed semi-structured interviews. While the literature provided the theoretical foundation necessary to identify trends and commonalities, there were knowledge gaps that prevented a detailed examination of the science-policy connection and the tools and mechanisms to forge stronger relations between the two disciplines, particularly in transboundary water areas. To address these gaps, interviews were carried out with lake scientists and policymakers and non-government participants to gain a more in-depth understanding of the science-policy interface in environmental policy processes and transboundary water management areas. Further I examined the observations gained through the literature review and interviews in the context of East Africa's Lake Victoria. Given that the management system is evolving, there is a modest amount of published literature available and the analyses were heavily informed by interview data.

A literature review is the most common initial research step available to researchers. Conducting a literature review is required to build the theoretical foundation and to reinforce the observations and findings of other forms of research, including interviews. To begin examining the science-policy interface in transboundary water management regimes, I reviewed peerreviewed scholarly literature and consulted grey literature and reports.

An abundance of literature is available on the interface between science and policy and is mainly published by academics and practitioners in government including agency scientists and policymakers (Brown, 2009; Keller, 2009; Graffy, 2008; Schaefer \& Bielak, 2006; Dale, 2001; Bradshaw \& Borschers, 2000; Lomas, 2000; Wynn \& Mayer, 1993). The references were not specific to transboundary water management but, rather, covered the broader scope of environmental policymaking generally and the integration of science specifically. The literature on transboundary water management extends beyond published papers and includes government 
documents such as annual and progress reports, proposals and project status reports, newspaper and magazine articles, websites and texts. Further, there are a number of agency and government websites providing information on existing transboundary water management regimes. A modicum of resources discussed the role of science in transboundary water.

The literature provided a strong understanding of the approaches for integrating science into the environmental policy process and highlighted some challenges associated with these concepts. Although there has been extensive research examining the science-policy interface, concluding the need to forge a stronger connection between the two, there have not been comparable studies examining how this can be achieved. Reviewing the literature on transboundary water management offered a sound understanding of the challenges inherent to collaborative management and identify some criteria for the development of effective transboundary water management systems, the majority of which could be influenced by science. However, as previously noted, there are areas where the literature fell short and required other forms of research to complement the information. Semi-structured interviews were conducted to address these gaps.

Semi-structured interviews are the middle-ground between unstructured and structured interviews, allowing the researcher to gain "detail, depth and an insider's perspective, while at the same time allowing hypothesis testing" (Leech, 2002, p. 665). I opted to conduct semistructured interviews as this style is most often used in elite interviews. They are also commonly used to interview senior government officials.

Telephone and face-to-face interviews were conducted with scientists, policymakers, technical advisors, and non-government participants both from North America and Uganda. Approval was obtained from the Ryerson Ethics Review Board to ensure that proper research 
protocols were followed. I conducted 25 interviews, of which 19 were face-to-face and the remaining on the telephone. Sixteen of those interviewed in-person were held in Uganda. While face-to-face interviews were prioritized, telephone interviews also provided valuable data. The suitability of telephone interviews is dependent on the research endeavour and consideration should be given to the level of sensitivity, access to hard-to-reach respondent groups, costs and interviewer safety (Sturges \& Hanrahan, 2004, p. 108). Conducting telephone interviews is appropriate when the researcher does not otherwise have access to the respondent; however, this approach deprives the researcher from observing the respondent's body language (Creswell, 1998; Rubin \& Rubin, 1995). Other researchers find telephone interviews an effective means of gathering data. Sturges and Hanrahan (2004, p. 115) concluded "telephone interviewing can be used successfully in qualitative projects" and in fact, "[b]y providing potential participants with a choice between telephone and face-to-face interviewing, a wider variety of respondents could be included and more information obtained...".

The selection of candidates was based on the literature search and professional contacts. The identity of the interviewees is kept anonymous throughout this paper. In cases where the interviewees' identities were disclosed, permission was obtained from the interviewees. Included in the appendix is the interview guide, which includes question themes and the consent form. These questions were adapted according to the candidates' expertise, organizational affiliation and involvement in environmental policy development.

Taking into account the advantages and disadvantages of the interview styles, face-toface interviews were carried out where possible. This included traveling to Uganda from MayJune 2009 for a period of four weeks to interview those involved with the management of Lake Victoria. Uganda was selected as the host country as it is home to a number of regional Lake 
Victoria environmental initiatives including the Lake Victoria Fisheries Organization (LVFO) and the Nile Basin Initiative (NBI). Further, of the East African countries, Uganda is the leader in environmental law and policy and has established organizations to build its scientific capacity such as the National Environment Management Authority (NEMA), National Fisheries Research Resources Institute and the Ministry of Water and Environment's Directorate for Water Resources Management (Gore, 2008). It also has the highest population growth rate in the region and its people are expected to becoming increasingly dependent on land, agriculture, and fishing including international fish exports as its primary commodity for their livelihoods (Bremner \& Zuehlke, 2009). Examining the evolution of the science-policy linkage in Uganda in this research paper is therefore an important element in understanding the connection between science and policy in the Lake Victoria management regime.

During that period I resided in Kampala, the country's capital city and where my academic affiliation, the Advocates Coalition for Development and Environment (ACODE), is located. I also traveled to the towns of Jinja and Entebbe to conduct interviews with lake scientists and managers. Two main organizations, the LVFO and National Fisheries Resources Research Organization (NaFIRRI), are located in Jinja. The Uganda Ministry of the Environment's Directorate of Water Resources Management, Global Water Partnership and Nile Basin Initiative are located in Entebbe. In situations where the respondent was inaccessible in person or preferred speaking by telephone due to scheduling conflicts, telephone interviews were conducted. 


\section{Case Selection}

Case studies are intended

to focus on a particular

issue, feature or unit of

analysis, rather than a

study of an entire system

or organization (Mohd

Noor, 2008). They are

particularly useful when

attempting to understand

an issue in great depth. To

gain a further understanding

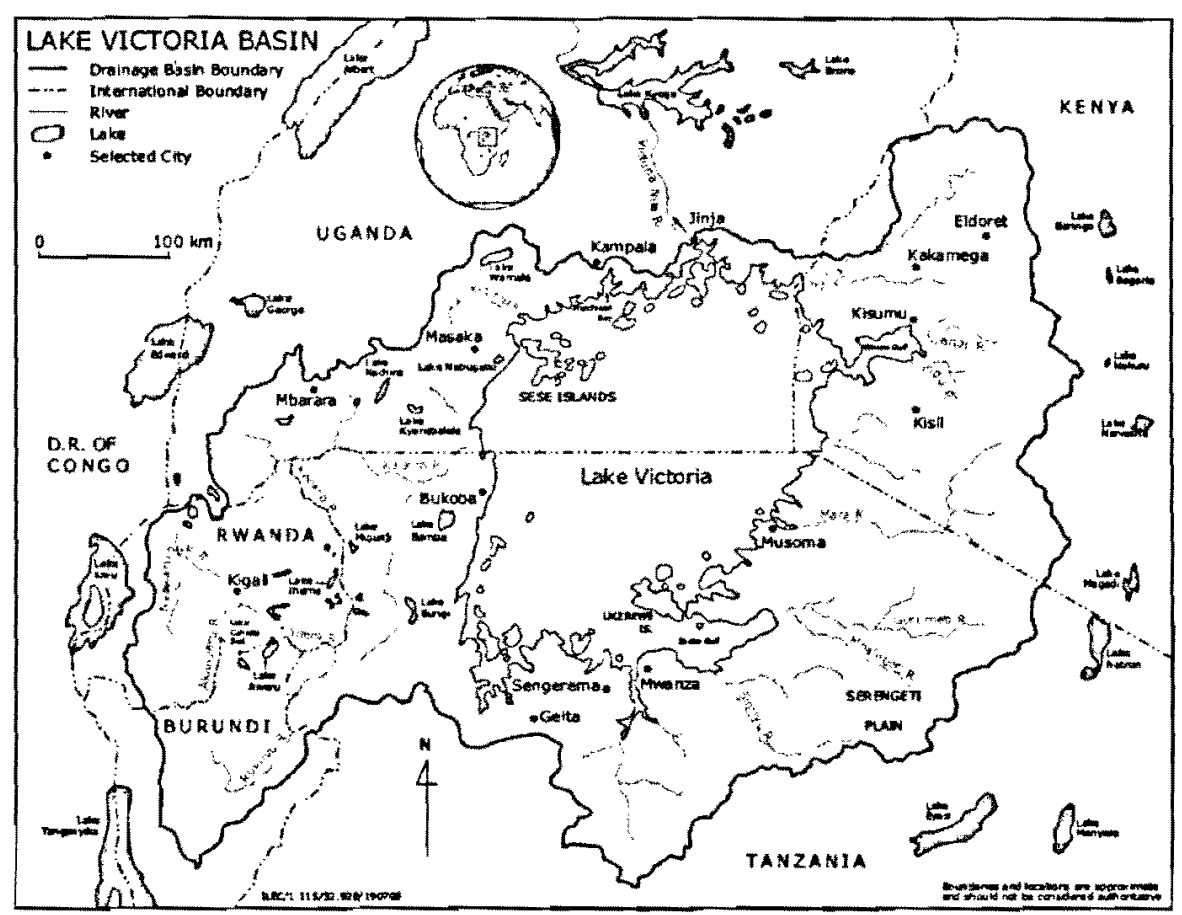

Figure 1: Map of Lake Victoria Basin

Source: Kayombo \& Jorgensen (2006)

of the science-policy

interface in transboundary water management regimes, a case study was conducted of East

Africa's Lake Victoria (Figure 1) to examine the causes for the divergence and the tools and mechanisms to narrow this gap in a practical context.

Lake Victoria is the second largest lake in the world by surface area and has experienced dramatic ecosystem changes over the past few decades. Although evidence suggests riparian countries have attempted to collaboratively manage the lake since the 1920 s, an effective and sustainable management regime has not yet been realized. Recognizing this void, bordering states, along with Rwanda and Burundi and the assistance of international partners have recently dedicated significant resources toward the development of an integrated water management system for the area. New coordinating mechanisms are being established as observed through 
the creation of the Lake Victoria commissions, and innovative tools are being developed and adopted including Beach Management Units. This is particularly significant as the lake is facing severe ecosystem challenges owing to increasing pressures on the resource. Basin countries require an effective and regional management system to address these challenges and to ensure the lake is utilized and managed sustainably for current and future generations. Given that the management regime is relatively young and management processes and practices are evolving, the key actors are presently addressing the challenges of establishing an effective transboundary water management system, including defining the role of science. Thus, the Lake Victoria management regime is an interesting example for examining science-policy linkages.

The case study was conducted by first performing a literature review to the extent that information was available to familiarize myself with the issues facing Lake Victoria and the existing management activities in the basin. To address knowledge gaps, semi-structured interviews were conducted with individuals involved with the management of Lake Victoria and specifically with those residing within Uganda given the location of my academic affiliation and contacts. The suite of respondents included scientists and policymakers in water quality and fisheries management, non-government organizations (NGOs), academics, donors and lending institutions such as the Global Environment Facility (GEF).

While the methods used in this project provided a sound understanding of transboundary water management, the science-policy interface and the instruments that could strengthen this connection, there are weaknesses in the approach that should be noted. Conducting a literature review is generally robust in that it allows the researcher to build a strong theoretical foundation on the subject matter. While "one of the most important uses of documents is to corroborate evidence" (Tellis, 1997, p. 8), there may also be biases in the selection of literature. This can be 
extended to the reporting of biases by the author. On a practical level, some literature may also be difficult to access and therefore, may be omitted from the final analyses.

Along the same lines, interviews can also be biased in the type of interviewees selected and the types of questions asked to the respondent. Tellis $(1997$, p. 8$)$ notes the potential for interviewee 'reflexivity', whereby the "interviewee expresses what [the] interviewer wants to hear." There are also criticisms of interviews in that the perspectives and opinions expressed are subjective and anecdotal in nature. Further, as the interviews were held with scientists, lake managers, academics, and NGOs residing in Uganda, there is a risk the responses may be biased towards a Ugandan perspective. Given the framework in which the data were acquired and examined, it is recognized the information provided could encompass an element of subjectivity. Future research with experts in Tanzania and Kenya would offer a more holistic and comprehensive view of the science-policy linkage in the Lake Victoria basin, as well as the level of scientific capacity in each of the riparian countries. The literature was used as a point of reference to the extent possible in efforts to minimize the degree of bias and information conveyed by interviewees was compared and contrasted with the literature and with other key informants.

Recognizing these weaknesses, I took precautions to minimize the biases that could potentially arise. With respect to the literature review, a number of sources were consulted to obtain varying perspectives on the subject matter and where possible, peer reviewed literature was used as a reference point. In structuring the interview phase of the study, however, my academic advisor and I used our professional contacts as a first step in acquiring interviewees to participate in the research. This eventually resulted in referrals to others involved with transboundary water management - sometimes referred to as the 'snowball method'. Although 
measures were taken to minimize biases by conducting interviews with a variety of individuals involved with transboundary water management and corroborating and comparing testimonials, it is accepted and understood that a complete elimination of bias in this research was unattainable due to a number of reasons including accessibility to interviewees, time and resource constraints, and the scope of project.

\subsection{Thesis Outline}

In the following chapters, this paper demonstrates that strengthening the science-policy connection is necessary for the development of effective policies and transboundary water management systems. It does so by first examining the interface between science and policy in environmental policy processes in Chapter Two. It is observed that policymakers generally adopt two predominant views when considering how to integrate science into a policy; one views scientists and their scientific knowledge as having functional value, while the other integrates science throughout the policy process to inform policy direction. This thesis then identifies some of the causes for the science-policy divergence stemming from these two approaches and highlight tools and mechanisms that can bridge the divide.

The complexities of transboundary water management are introduced in Chapter Three. Transboundary water areas are experiencing increasing demands owing to growing populations and economies and require effective management practices to ensure the sustainability of the resource and its ability to support current and future anthropogenic activities. The paper discusses the role of key actors in these areas and highlights criteria for the development of effective management systems. It observes that science can influence the achievement of these criteria. Further this dynamic and the role of science in transboundary water management are 
examined in Chapter Four. The causes for the science-policy disconnect are explored and tools and mechanisms to strengthen the science-policy interface in transboundary areas are discussed.

In Chapter Five, the observations and conclusions gained from these discussions are then examined in the context of Lake Victoria. Lake Victoria's management regime is emerging but the linkages between science and policy are not yet well defined. This analysis reveals how the approach to management in the Lake Victoria Basin is shifting towards an integrated framework, one that requires a stronger connection between science and policy. This paper explores the various tools and mechanisms adopted by lake scientists and managers and observe that significant efforts and progress have been made to forge stronger relations between the two communities.

In Chapter Six, the thesis concludes by confirming that strengthening the science-policy interface is critical for the development of effective environmental policies and enhancing policy credibility. It is highlighted that this is particularly relevant in areas of shared waters where the magnitude of the issues and the number of key actors involved can add another layer of complexity. A strong science-policy connection can alleviate some of the challenges inherent to collaborative management. And finally, this paper offers recommendations to transboundary water managers, scientists and decision-makers that can reinforce the science-policy interface and contribute to more effective transboundary management practices and policies. 


\section{Chapter 2: The Science-Policy Interface}

\section{Introduction}

The policy realm is confounded by 'multiple streams of activity'; only when confluent, can they influence policy direction and outcomes (Keller, 2009; Kingdon, 1995). The convergence of these streams of activity can connect ideas, solutions and political will. This can be further described as those rare moments when a 'policy window' opens; when useable knowledge resonates with other contextual factors, and the "constellations of values may happen to coincide with the research's implications" (Lomas, 2000, p. 142).

This observation serves that policy development processes do not follow linear paths (Keller, 2009, p. 6). In the case of environmental policies, processes require policymakers to maneuver through various facets of ecological, socio-economic and political issues to produce outcomes. Their decisions at particular junctures throughout this journey influence the direction and eventual outcome of policies. The decision to include for example, science experts and science knowledge, and the point at which science is 'engaged' may have the potential to influence policy direction and outcomes.

It is in this context that the interaction between science and policy, the science-policy interface, is important. The science-policy interface can be understood as the moments and/or opportunities in a policy process when scientific evidence or individuals bearing that evidence are engaged or integrated. This paper reviews the arguments offered for strengthening this interface in order to develop effective policies. It does this by first exploring the role of science in environmental policy development, including the policy venues in which science can be engaged. The thesis also examines the implications of equating science with objectivity and the weight this understanding could have on policy positions. This is followed by a discussion on 
the perpetual divergence between social and natural sciences - a matter of increasing importance in policy development observed through the growing literature examining the issue. The potential causes and consequences that can result from the science-policy gap are highlighted and various tools and mechanisms for bridging this divide are identified. This thesis argues that the adoption of instruments, including those that enhance communication and stakeholder engagement, and break down disciplinary 'silos', into management systems will narrow the divergence between science and policy, and will contribute to the development of effective environmental policies. Doing this will set the stage for the examination of the science-policy interface in transboundary water management regimes.

\subsection{Science in the Environmental Policy Development Process}

There are various points in the policy development process where science can be integrated. Science is generally defined as "any system of knowledge that is concerned with the physical world and its phenomena and that entails unbiased observations and systematic experimentation. In general, science involves a pursuit of knowledge covering general truths or the operations of fundamental laws" (Encyclopedia Britannica, 2009). In this thesis, science refers to this scientific knowledge, as well as the generators of this knowledge - scientists - and indigenous knowledge. Scientists are individuals that often invoke a body of scientific knowledge as the basis for their policy claims (Keller, 2009, p. 16). Indigenous knowledge is local knowledge that is unique to a given culture or society and is the basis for local-level decision-making in activities including agriculture and natural resource management in rural communities (World Bank, 2009). Scientists, science expertise and indigenous knowledge can and do inform environmental policy choices. I recognize that scientists and policymakers are not mutually exclusive and that in many cases, scientific and technical experts can inhabit both science and 
policy realms. However, for the purposes of this research, scientists and policymakers are viewed as distinct actors in the environmental policy process, each having independent roles and functions in policy development.

The point at which science interfaces with policy can determine its role in the development process. I use Graffy's (2008) description of the policy process, which includes five stages: (1) issues emerge; (2) issues are framed or assigned meaning; (3) priorities are set; (4) priorities are legislated; and (5) goals are implemented. This is expanded to include an additional component of evaluation, which is often included in other models. While introducing a six-stage process suggests a linear approach to policy development, it should be noted that this simplification of the process is used to structure the discussion on the utility of science and the mechanisms that will be identified to address the challenges of integrating science into policy processes. It is recognized that policy development rarely follows a linear path. Stages are typically blurred and more iterative than what is presented.

The perspectives held by policymakers on the role of science can shape the extent policy interfaces with science. Keller (2009) identifies two predominant views on science in environmental policy development: rationalist and logical positivist ('positivist'). These two perspectives define the role of science and identify the policy venues in which science could be integrated. While it is recognized that differing perspectives on what is defined as rationalist and positivist, this paper only refers to Keller's (2009) description on integrating science into the environmental policy process.

\section{Rationalist}

The rationalist conception of science can be best described as connecting 'means with ends'. In this view, the role of science is to assist policymakers in achieving their intended objectives and 
is deemed successful when it enables policymakers to choose a policy solution that (a) brings them closer to their stated goals; and (b) outperforms other solutions in achieving those goals (Keller, 2009). Science is "merely used as an aid in finding the most effective, efficient means to achieve [the policy goals]" (Keller, 2009, p. 29). The rationalist view of science in policymaking clearly demarcates the boundary between science and policy. Science in this view could be likened to a map and the policymaker as the traveler; where a traveler's origin and destination are pre-determined - the policy objective - and the map is the tool that delineates a path to arrive at the intended location. The rationalist view therefore confines the role of science to a policy tool that produces and supports solutions. It stipulates that science should not help policymakers define their objectives - science is integrated after the policy goal is established.

Jonathan Lomas (2000) observes the prevalence of the rationalist view in current policy processes. Scientific researchers and policymakers tend only to connect if they connect at all around the products of their processes. Lomas argues that by treating policy as a discrete product, researchers miss and/or are left out of the opportunities to influence how policy issues are framed - the intent of the rationalist approach.

This view suggests scientific integration at the definition stage of policy processes risks undermining the ability of science to provide reliable and valid information. By creating a boundary between science and policy, the rationalist approach preserves the norms of the science and policy disciplines (Keller, 2009, p. 28), and therefore serves to protect science from political bias. Scientific research can proceed in the absence of political direction. The rationalist approach also limits the extent of technocracy - an over-reliance on technicians and technology by governments in managing society - by formalizing and controlling the degree of integration. Given that the rationalist theory clearly draws a distinction between science and policy, it can be 
argued that the rationalist view reinforces the divergence between science and policy. This notion will be explored in greater depth later in the chapter.

\section{Positivist}

The positivist approach to policy development is more fluid than its rationalist counterpart. The positivist view of science allows for scientific integration at various stages throughout the policy life cycle, including the definition stage. The positivist approach does not view the inclusion of science in setting policy objectives as a risk but rather, a mechanism for informing policy goals. It holds that science has the potential to resolve policy controversies and does so by invoking "science as a prerequisite for policy debate such that science defines the terrain that is factual and uses that to circumscribe issues that remain open for debate" (Keller, 2009, p. 30). Contrary to the rationalist view, the positivist theory can serve as the basis for bridging the science-policy gap.

Keller (2009) also describes a softer version of the logical positivist view that assumes science does not eliminate the value conflict between science and policy but instead limits the conflict in scope. This 'soft-positivist' approach proposes that conducting scientific research is an important preliminary step in resolving policy controversies. This is often demonstrated in the realm of environmental policy development where discordant ecological, socio-economic and political values can result in conflict.

The rationalist and positivist conceptions both view science as a resource in the policy process. Where they differ is the point at which science is integrated into the policy life cycle and the approach taken to address the issue of technocracy: "At issue is the power that actors claiming scientific insight have [in] shap[ing] policy debates in terms that encourage reliance on scientists or science in shaping policy outcomes" (Keller, 2009, p. 31). The positivist view 
supports the integration of science throughout the policy process and emphasizes the role of science in setting policy objectives and mitigating policy controversies. The rationalist theory formalizes the venues in which science is integrated into the policy process. This approach delineates science and policy as distinct entities and utilizes science as a vehicle to attain predetermined policy objectives.

The issue of technocracy is the crux of the debate between the rationalist and positivist views. I include here a brief discussion on the notion of equating science with objectivity and how this power can influence the development of environmental policies. This leads to important questions about the degree of technocratic authority in policy regimes.

Wynn and Mayer (1993) observe that our policy culture has evolved to become 'scientistic', one that gives credibility to opinion only when it is defined in scientific language. Scientists present their knowledge and themselves as objective, devoid of any subjectivity, (Brown, 2009, p. 168), which refrain them from making judgments about policy (Keller, 2009, p. 12). This idealized image of science has given rise to increased technocratic authority in the policy realm and further legitimizes the role of science in the policy process. This may be especially true in domains such as environmental policy, where participants view scientific and technical information as central (Keller, 2009, p. 43). In these circumstances, scientific expertise can be used to bolster policy positions (Keller, 2009, p. 16).

Given these observations, one may conclude that science could potentially dominate the policy arena, particularly in the environmental realm. In actual fact, what is frequently observed is a disconnect between science and policy. In some cases, the consideration of science is an afterthought. In the next section, this paper explores this disconnect and attempts to identify the causes that have perpetuated this divergence. 


\subsection{The Divergence Between Science and Policy}

Given the wealth of scientific evidence available to policymakers, it is perplexing that science is not readily integrated into the policymaking process (Dale, 2001, p. 99). The rationalist and positivist theories offer two distinct ways that policymakers can view the utility of scientific experts and expertise. The degree that science interfaces with policy therefore varies and is dependent on how policymakers privilege it as a tool to meet pre-determined goals or allow it to influence and frame the goals themselves. Given these variations, there is the potential for science and policy to diverge along different tracks. Figure 2 illustrates how the science-policy interface can be weakened or strengthened as a result of the approach taken to integrate science into the policy process. It also identifies the potential causes contributing to the widening and perpetuation of the divergence. Similarly, the figure depicts ways in which the divide can be narrowed, forging a stronger connection between science and policy. These concepts will be discussed later in this chapter.

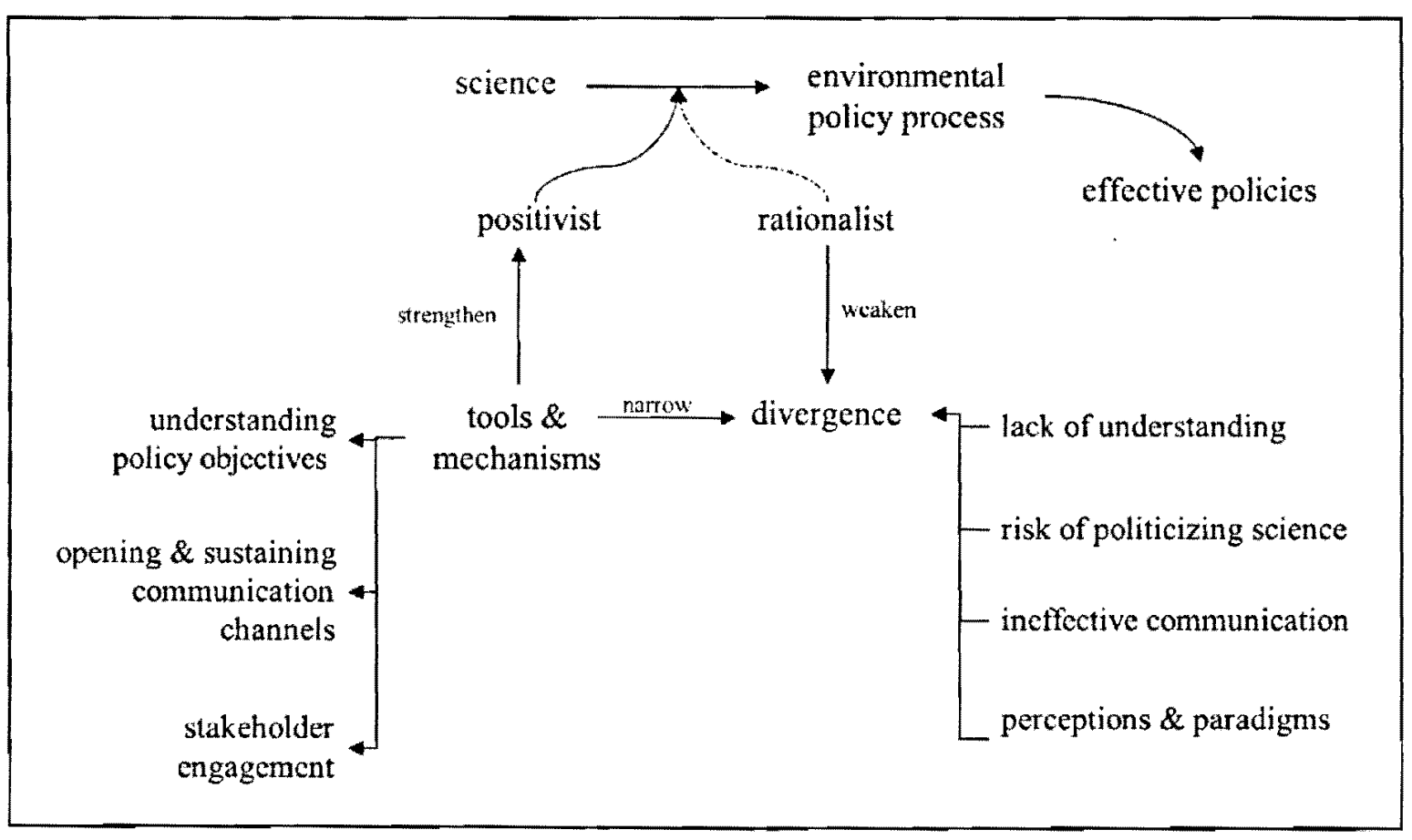

Figure 2: Integrating science into the environmental policy process 
Mark Brown (2009, p. 163) notes modern societies have conceptualized science and politics as largely insulated from each other. The decision to view them separately has widened the gap between science and policy and is reflective of the rationalist view. I take this opportunity to explore the causes for the divergence and observe that the basis for the gap is largely rooted in the lack of understanding of disciplinary cultures, processes and objectives; existing perceptions and paradigms; the risk of politicizing science; and lastly and perhaps the underlying issue, the failure to communicate effectively. These observations are important for the future discussion of transboundary water management regimes generally and Lake Victoria specifically, where the causes for the science-policy divide could potentially be exacerbated owing to the complexities of multilateral collaboration.

\section{Lack of Understanding of Disciplinary Cultures, Objectives and Processes}

What brings people together is their affinity for similar attitudes, beliefs and practices. Having a particular set of beliefs and attitudes can establish cultural trends among individuals and even within disciplines. On the contrary, diversity between individuals can sometimes result in a separation, leading to an unwillingness to understand and accept alternative perspectives and ideas. This reluctance is evident in the world of science and policy, where philosophical boundaries between the disciplines rely on stereotypical views (Brown, 2009, p. 166).

Disciplinary cultural differences can influence the degree information is shared by scientists and received by policymakers. Dale (2001, p. 113) observes that managers and scientists live and work in vastly different cultures. As a result, they often view the world from very different perspectives and act on the basis of different values. What is deemed important to scientists may not resonate with their policy counterparts. The opposite is also true. The meaning of potentially useful information for example, can diverge widely between the two 
groups leading to policy paralysis as a result of what is perceived to be inaccurate or insufficient information (Dale, 2001, p. 113). Thus, the commonly assumed linear relationship between science and policy - that is, science is disseminated to policymakers for application in policy development - is in fact, much more complex and convoluted.

The core assumptions of science and policy are also fundamentally different. Science strives to increase our understanding of the natural world. The process by which it attempts to replicate natural functions is through the design of set methodologies that produce outcomes that are empirically justified. On the contrary, policy sets out to establish a set of 'guiding principles' - goals and programs - to provide order within organizational settings and to produce outcomes that are shaped by desires and ideologies; they are a function and result of compromise between many actors. Policy is much more normative and does not travel along linear paths but rather takes into consideration issues of social and political importance, which are more often than not subjective and difficult to quantify (Cahn, 2008).

The lack of understanding and appreciation of the processes required for the production of scientific knowledge and policy can widen the gap between science and policy. As previously mentioned, the environmental policy process requires the consideration of ecological, socioeconomic and political issues and implications. Given that these elements are somewhat dynamic, policy processes can often be irrational and non-linear and are a cause of frustration for scientists, whose objectives and paths are much more narrowly focused (Anderson, 2007). Lomas (2000, p. 140) states, "researchers need to appreciate that decision-making is not so much an event as it is a diffuse, haphazard and somewhat volatile process."

The process by which scientific knowledge is produced is often iterative, lengthy and bounded by limits and uncertainties as well. Policymakers can experience frustration when they 
discover policy goals may not be arrived at in a straightforward and timely manner as a result of insufficient information (Anderson, 2007). They do not recognize or appreciate the numerous stages involved with scientific research and view 'research as a retail store'. By this, Lomas (2000) suggests policymakers view science as products on shelves, waiting to be selected to advance policy goals.

Attaining a policy objective requires all relevant actors to understand the intended goal and the role they play in achieving it. In many situations, the research results are not utilized in the policy process because the results do not address the issue at hand. It became evident through interviews that a hindrance to effectively integrating science into policy development is the incessant practice of conducting research within silos and the lack of understanding of policy needs. For example, Karl Schaefer, Head of Strategic Science-Policy at Environment Canada suggested that more investment is required to understand the science needs and preferences of the science users and practitioners: "Nobody is asking [policymakers] how they want their science, when they want it, in what format [they want it] to optimally inform decision-making. There is no substitute for asking the user what they want and how they want it if you want to optimally inform decision-making" (Interview, March 25, 2009, Toronto, Canada). Professor Frank Kansiime at the Institute of Environment and Natural Resources at Makerere University in Uganda supports this position. Professor Kansiime shared that academics have in the past conducted research without involving policymakers in the initial stages of research design. Research outcomes did not complement policy agendas and were therefore impracticable. Professor Kansiime suggested that academics could take a proactive approach by involving stakeholders during the initial stages to identify research areas most relevant to science and policy formulation (Interview, June 8, 2009, Kampala, Uganda). A Great Lakes policy expert 
also noted that there are many issues within an ecosystem and scientists cannot know everything. Policymakers can tell the scientists what the priorities are, and the scientists can then put their effort into those (Interview, April 24, 2009, Toronto, Canada). It is important to note that producing policy-relevant research has the potential to politicize scientific research and the scientific process. Scientists and policymakers must be cognizant of this risk and to ensure that research is 'prioritized' and not 'politicized'.

The opposite has also been observed where the policymaker fails to ask the right questions to the scientist. As one Great Lakes policy expert noted, if policymakers are science illiterate they may ask the wrong questions, which will lead to the wrong answers (Interview, April 24, 2009, Toronto, Canada). In these instances, policymakers may suffer from paralysis or take action for the sake of taking action or responding to political pressure.

Bradshaw and Borchers (2000) characterize the differences between science and government institutions with respect to desired goals, acceptance of risk and intended audiences (see Table 1). These distinct attributes can influence the degree scientists and policymakers interact.

Table 1: Characteristics of Science and Government Institutions

\begin{tabular}{|c|c|}
\hline Science & Government \\
\hline probability accepted & certainty desired \\
\hline inequality is a fact & equality desired \\
\hline anticipatory & time ends at next election \\
\hline flexibility & rigidity \\
\hline problem oriented & service oriented \\
\hline discovery oriented & mission oriented \\
\hline failure and risk accepted & failure and risk intolerable \\
\hline innovation prized & innovation suspect \\
\hline replication essential for belief & beliefs are situational \\
\hline clientele diffuse, diverse, or not present & clientele specific, immediate and insistent \\
\hline
\end{tabular}

*adapted from Bradshaw \& Borchers, 2000 
From this table, one can observe that attributes of science and government are inherently different. These differences may lead scientists and policymakers on opposing tracks while working towards their respective goals. Becoming familiar with the differences in disciplinary cultures and processes could allow science to become better integrated into the policy development process. A lack of understanding between the science and policy communities can breed mistrust and discourage cooperation. The science-policy interface cannot be strengthened without some element of trust between communities and understanding of differences. Cultural differences can be further exacerbated if the values, ideologies and perceptions of the world are incompatible.

\section{Perceptions and Paradigms}

The divergence between science and policy can also be perpetuated by the disciplinary philosophical and theoretical frameworks or paradigms held by scientists and policymakers. The values and ideologies acquired by these communities construct formidable structures that make adapting to new information problematic. The inherent and interlocking values and ideologies can prevent even the uptake of new information (Dale, 2001, p. 100). As one scientist stated: "it is difficult for [policymakers] to change what they have already decided" (Interview, May 26, 2009, Jinja, Uganda). For example, natural resource exploitation has traditionally dominated anthropogenic interactions with the environment. The conventional concept of development means continually expanding industrialization, economic growth, and higher levels of consumption and production, with consequent environmental and health impacts accepted as unfortunate costs outweighed by the gains (ANPED, 2006). The structural barriers created as a result of this industrial paradigm reduce the ability and the capacity of new concepts and alternative models to challenge the dominant paradigm (Dale, 2001, p. 100). In fact, 
organizational structures and programs have the potential to calcify existing paradigms: "current economic activities are encouraged through government programs and incentives that result in continued exploitation of natural resources" (Dale, 2001, p. 102).

This observation is illustrated in organizational structures that govern fisheries in Lake Victoria for example. It was suggested by one fisheries scientist that policymakers have a vested interest in maintaining the status quo and continuing with existing programs and are therefore resistant to institutional changes (Interview, May 26, 2009, Jinja, Uganda). As such, there is an inability to respond to new information even when the facts dictate the potential for tragic and irreversible consequences. A number of Lake Victoria researchers noted that in the past, it would be impossible to suggest seasonal closures for fisheries or to recommend the banning of fishing equipment. Such recommendations would contradict the policy objective of expanding the fisheries sector for economic development and poverty reduction. In this example, the desire to exploit the fisheries resource for economic gain prevented the uptake of scientific information and interfered with the ability of science to inform policy: "when one is stuck in a spiraling pattern of exploitation and conservation, systemic learning cannot take place and reactive rather than proactive policy choices become the norm" (Dale, 2001, p. 108). The impending collapse of the Nile perch and tilapia fisheries eventually necessitated the development of policies to ban illegal fishing equipment. At that point, science was engaged in the policy process. The point at which science interfaced with policy reflects a rationalist approach to policy development.

The concept of 'received wisdom' or 'narratives' as introduced by Leach and Mearns (1996) can also be linked to the construct of paradigms and can indirectly widen the gap between science and policy. 'Received wisdom' is defined as information that has been accepted as the truth with no consideration given to the information's validity or relevancy in the current 
context. In Ronald Wright's $A$ History of Progress (2004), the author observed that a 'snapshot in time' has the potential to perpetuate over decades if alternative information is not available to challenge that reality. I argue that even when new information is available, this 'received wisdom' can continue to strengthen existing paradigms and prevent the acceptance of new information that may refute the dominant ideology or policy position. For example, the perception that point sources of pollution are the most significant and sole contributors to the deterioration of international lakes and rivers continues to dominate environmental policy development in some areas. While large point sources of emissions do contribute to elevated pollution levels, non-point sources such as unsustainable agricultural practices and urban runoff could also considerably adversely affect water quality. A shift in the perception of addressing point and non-point sources of emissions is required for future water management. Failing to do so risks the development of flawed or misguided policies.

Narratives that have perpetuated over time and that have been used as the basis for policies are difficult to challenge when institutionalized; that is, when narratives lead to the creation or strengthening of organizational structures and is used as the foundation for the norms and rules of organizational activities. Put differently, the information has become entrenched in an organization. The consequence of institutionalized knowledge is the inability to receive new information, as it may be perceived as a threat. The more institutionalized the information, the more it "becomes embedded in machines and other material artifacts, [and] the more stable [it] become[s]" (Brown, 2009, p. 175).

While the persistence of paradigms has successfully widened the gap between the two groups in the past, there is evidence that paradigms are shifting to an integrated approach to environmental management and consequently influencing the degree of interaction among 
scientists and policymakers. Management approaches are evolving from single ecosystems to a more integrated system. This evolution in management approaches will be discussed in the next chapter in the context of transboundary water management. However, there remain other important barriers to science-policy integration. One such barrier is when science becomes politicized; that is, the evidence is used or manipulated for political gain.

\section{Politicization of Science}

Keller (2009, p. 27) observes that the politicization of science occurs when interests of individuals and/or groups introduce bias into scientific work or the representation of their work in policy settings. The rationalist view demarcates a boundary between science and policy and suggests this distinction protects science from politicization and the policymaking process from technocracy. However, even as modern societies attempt to conceptualize science and politics as largely insulated from each other (Brown, 2009, p. 163), it does not prevent science from being politicized.

As one senior water manager noted, "all the decisions we [make] as scientists are for politicians. Anything technical is [therefore] political" (June 11, 2009, Entebbe, Uganda). This supports Bocking's (2006, p. 33-44) observation that science is widely viewed as too closely tied to powerful interests, especially industry and government. Given that there is a perception that science can be used to advance political agendas scientists fear that actively engaging or participating in policymaking in any way would appear to be a discarding of objectivity and could potentially result in a loss of credibility with their scientific peers (Graffy, 2008). In these circumstances, scientists may distance themselves from the policy process, which strengthens the divergence. The need for open communication and trust is therefore reinforced. 


\section{Communication Challenges}

Given the inherent differences in cultures, processes and objectives, it is not surprising that ineffective communication plagues the science-policy interface, often causing the rift between scientists and policymakers to widen. The issue of 'translating' science into useable information for policymakers was highlighted in the literature and by interviewees as a fundamental communication obstacle.

When asked to identify challenges with the integration of science in policy development, a number of interviewees independently highlighted communication, and specifically the challenge of translating science into an understandable format for non-technical audiences. The interviewees were of the mind that most scientists are not trained to disseminate technical information into usable formats for non-technical users to integrate into their work. Scientists do not have as yet, a clear system of communicating their results and the consequences of the results to those responsible or involved with policy and decision-making, or to those in departments or agencies responsible for implementing activities. "Scientists are not very good at communicating outcomes in a way that is understandable by the general public, and certainly by politicians" (John Metzger, Water Resources Management Adviser, Directorate of Water Resources Management, Uganda Ministry of Water and Environment, June 11, 2009, Entebbe, Uganda).

It is the perspective of some policy experts that "academics and researchers tend to be confined to their ivory towers" (Interview, June 11, 2009, Entebbe, Uganda). Scientists equate being asked to respond to policy needs as being asked to 'dumb down' the science and can at times be reluctant to do so (Graffy, 2008). On the other hand, policymakers do not have the expertise or time to digest raw scientific information. The barriers to effective communication 
and a lack of regular interpersonal interaction between scientists and policy staff can therefore foster mutual belief in stereotypes that discourage collaboration (Graffy, 2008). As previously mentioned, these stereotypes can draw philosophical boundaries between science and policy.

As revealed in Table 1, the inherent differences between science and government can prevent science from being integrated effectively into the policy process. Bradshaw and Borchers (2000) suggest governments desire certainty, a necessary element to minimize risks and probability of failure. However, the very nature that scientific information can be provisional has the ability to further divide the communities. As one respondent noted, "science does not give you absolute certainty, and leaves you with a lot of hanging questions. You can see [then that] science [and] policy diverge quite considerably" (Interview, March 27, 2009, Toronto, Canada). The way in which uncertainty is communicated to policymakers can therefore influence the extent that science is integrated.

Scientific uncertainty can arise out of a number of circumstances; from the selection of methodology to the degree that scientific consensus can be reached uncertainty can limit the utility of information. The reported discrepancy among scientists at the Climatic Research Unit (CRU) at the University of East Anglia (UEA) only weeks prior to the 2009 United Nations Climate Change Conference $\left(15^{\text {th }}\right.$ Conference of the Parties - 'COP $\left.15^{\text {' }}\right)$, illustrates the influence divergent views can have on policy agendas and public confidence in science (The Daily Telegraph, December 6, 2009; The Globe and Mail, December 11, 2009; Macleans, January 7, 2010). The presence of scientific uncertainty and discrepancy could be interpreted as undermining scientific authority (Bradshaw \& Borchers, 2000).

While science is never absolute, "there is a huge difference between being informed by the latest available science versus just flailing around" (Interview, April 24, 2009, Toronto, 
Canada); that is, although there may be uncertainty with scientific information, policymakers would still benefit from being informed by available scientific information rather than excluding it altogether. To minimize the effects of uncertainty, one must exercise judgment and look at the weight of evidence to make informed policy decisions. However, the challenge remains of translating scientific uncertainty into guidance for practical action. Wynne and Mayer (1993) observed that uncertainty has typically been left to scientists to address. Given the potential implications of scientific uncertainty on society and politicians, a broader discussion with scientists and policymakers needs to be held to better articulate the uncertainties in scientific findings. Policymakers and politicians need to recognize this as a central part of the scientific process and adapt policymaking to this uncertainty.

The seemingly strong divide between science and policy can be attributed to the fundamental differences in cultures, processes and objectives. This is compounded by organizational structures developed in response to existing paradigms, as observed with natural resource exploitation. The issues of politicization of science and ineffective communication can further impede the uptake of information by policymakers and the willingness of scientists to share information during the policy process. Potential consequences of this divergence can include the development of ineffective or erroneous policies that produce undesirable results. The failure to integrate science effectively, if at all in the policy process could also delay the policy process generally.

Given that a weak science-policy interface has the potential to debilitate policies and encumber policy development it is desirable to strengthen this interface by defining tools and mechanisms that could narrow the science-policy gap. The next section identifies some ideas and instruments for this purpose. 


\subsection{Strengthening the Interface}

The way in which the relationship between science and policy is conceptualized holds important implications for practice (Graffy, 2008). Science can affect policy outcomes and therefore has the potential to create lasting effects on intended recipients. It is on this basis that I argue that the pursuit of evidence-based policies is an act that is both necessary and responsible. An evidence-based policy approach is defined as one that "helps people make well-informed decisions about policies... by putting the best available evidence from research at the heart of policy development and implementation" (Davies, 2004, p. 3). Forging a strong connection between science and policy allows for better scientific integration into policy frameworks. This increases the likelihood of policies being informed by science, which enhances credibility and transparency. Decision-making is better when informed by the latest scientific information (Schaefer \& Bielak, 2006).

Even though the challenges associated with the science-policy interface appear overwhelming all of the experts interviewed emphasized the need to narrow the divergence and highlighted the importance of a strong science-policy relationship: "science is the fundamental underpinning to policy development" (Interview, March 25, 2009, Toronto, Canada). A number of scientists and policy experts observed that science and policy complement one another: One is helping the other...they are two helping hands. It is a give and take... an iterative process between policy and science. This observation is also reflected in the literature. Brown (2009, p. 178) concluded that neither scientific nor political representation can do without the other and that it is right to treat them in tandem.

Adopting a positivist view to policy development can strengthen the science-policy interface. Lomas (2000, p. 142) suggests "better links between research and decision-making 
depend... on the two communities finding points of exchange at more than the 'product' stages of each of the processes". This view is supported by a number of interviewees. For example, it was noted that the starting place for policy should be at the very least a survey and analysis of available science. Further, the lessons learned from previous applications of science can help inform the future direction of policies (Waiswa Ayazika, Environmental Impact Assessment Coordinator, NEMA, June 1, 2009, Kampala, Uganda).

While the literature on the science-policy divergence suggests researchers and policymakers could both benefit from greater understanding of each other's worlds, and consistently concludes that more effort is required to create structures or mechanisms to bring them together, little has been done to identify mechanisms for this purpose (Schaefer \& Bielak, 2006). I take this opportunity to use the literature and interviews to identify instruments that could narrow the gap between science and policy. Some tools could be adopted and implemented quite easily to facilitate communication between scientists and policymakers. Other mechanisms require paradigm shifts and institutional modifications - instruments that require much longer timeframes and sustained leadership and are therefore more prone to resistance and difficult to execute. With the identification of these mechanisms, it is then possible to evaluate their potential application in specific cases.

The strength and persistence of disciplinary silos have contributed to the widening of the science-policy gap and resulted in inefficiencies in both policy processes and products. As Lomas (2000) observed, researchers and policymakers tend to only connect around the products of their processes. Schaefer (Interview, March 25, 2009, Toronto, Canada) and Kansiime (Interview, June 8, 2009, Kampala, Uganda) recognize the deficiencies with this practice given that products developed within silos risk being futile. 
While the breaking down of these silos is a means to strengthen the science-policy interface, it may require an overhaul of existing institutional structures and paradigms - not an easy feat. There are, however, tools that can be adopted to help pave the way. I posit that the utility of science products could be enhanced if knowledge producers actively seek to understand policy objectives. Further, opening and sustaining communication channels between scientists and policymakers can alleviate misconceptions and increase understanding. To illustrate this, I identify and discuss communication tools that have been adopted by government institutions. Lastly, I discuss the value of stakeholder engagement to strengthen the science-policy connection.

\section{Understanding Policy Objectives}

Scientists' lack of understanding of policy goals and the process itself is a barrier to effectively integrate science into the policy process. As previously indicated, there has traditionally been a practice of conducting research without giving consideration to how that information will be utilized. "The old paradigm where science produces a piece of work that is then passed off to a policy person needs to be replaced with a new paradigm" (Schaefer \& Bielak, 2006, p. 432). What is required for policy-relevant research is for policymakers to help scientists 'prioritize' scientific research.

Increased understanding of research needs and preferences of science users can contribute to a new paradigm where regular and frequent interaction between researchers and policymakers is the norm. Graffy's (2008) proposed Functions of Scientific Information Model (FOSI) (table 2) could be adopted to facilitate this process. The model breaks down assumptions and stereotypes and opens dialogue about what scientists are already doing and how they can further enhance policy relevance. 
Table 2: Functions of Scientific Information Model (FOSI) Linking Science and Policy

\begin{tabular}{|c|c|}
\hline Stages of Policy Process & Corollary Functions of Science Information \\
\hline issues emerge & announce discoveries \\
\hline frame issues & put issues into perspective \\
\hline set priorities & test decision options and scenarios \\
\hline legislate priorities/goals & validate choices or trade-offs \\
\hline implement goals & enable implementation \\
\hline
\end{tabular}

*adapted from Graffy, 2008

In this model, scientific information complements the policy process by framing policy options in a realistic setting. The premise for the model relies on scientists being engaged during the early stages of the policy process, the point at which policy objectives are being defined. This allows researchers to apply their science expertise when issues emerge and is reflective of the positivist approach. Science also has the role of validating policy choices and enabling implementation. Through this early and ongoing involvement, researchers can then prioritize their studies with the objective to address the most relevant policy goals. The opposite is also true in that scientists can also announce discoveries to guide policy direction. It could be observed then that the FOSI model takes a positivist approach to environmental policy development.

\section{Opening and Sustaining Communication Channels}

The degree that science is utilized in the policy process is contingent upon the ability of its audience to understand it. There was overwhelming agreement by those interviewed that the communication or 'translation' of scientific information to non-technical audiences is the biggest challenge facing the uptake of policy-relevant research. "Describing [an] issue in the staid technical language typical of scientific publications" is much less effective than describing the same issue using "evocative language to support...arguments" (Keller, 2009, p. 1). The global 
interest in the climate crisis was in part the scientists' ability to act "more like political advocates than like scientists" (Keller, 2009, p. 1).

I previously indicated that disseminating technical information to science users has traditionally been missing from the scientists' repertoire of tasks. Researchers lack the training to communicate or translate information into usable formats for users of science. The concept of 'knowledge brokering' is proposed to address this issue. Enhancing communication channels is not however the sole responsibility of scientists but also requires the efforts of policy experts. The participation of researchers and policymakers in generating usable and effective communication materials, sustaining dialogue and creating information exchange opportunities is essential for science to inform policy. Lastly, other stakeholders such as non-government organizations can also help communicate science to its users.

When a policy window opens, policymakers must act quickly to seize the opportunity to influence policy direction and outcomes before the window closes. Disseminating the right information to the right people in a timely fashion is essential in the policy realm (Karl Schaefer, Head - Strategic Science-Policy, Environment Canada, March 25, 2009, Toronto, Canada). Moreover, policymakers not only lack the time, it is highly probable they also lack the literacy to turn scientific findings into good public policy (Keller, 2009, p. 17). Knowledge brokering is a tool that can be integrated into policy structures to help satisfy the demands of a fast-paced policy culture. A knowledge broker is defined as a low-to-middle level bureaucrat who has a knack for framing science in policy-relevant terms and is institutionally well positioned to make such arguments (Keller, 2009, p. 17).

The work of a knowledge broker can, in effect, lead to an overall efficiency of the policy process by translating technical information into products that could easily be interpreted and 
applied. In situations where science is regularly communicated to policy and program managers, the information could be used to define the policy goal or create the impetus to explore specific policy paths. At minimum, this information could be used to inform policy managers of the state of knowledge that is pertinent to their issue. Science products in the form of reports, fact sheets and newsletters are simple ways to communicate research to the 'informed public', defined as the decision-making audience including those that develop policies and manage programs (Karl Schaefer, Head - Strategic Science-Policy, Environment Canada, March 25, 2009, Toronto, Canada). Knowledge brokers also strive to improve the accessibility of scientific information through tools such as websites.

For example, the Canadian government's desire to strengthen the research and role of the Canadian National Water Research Institute (CNWRI) led the nation's Department of Environment to modify its organizational structure. It created a dedicated liaison branch, the Science and Technology Branch. The branch's mandate is to strengthen science-policy linkages by functioning as the department's knowledge broker. Staff undertake activities that broker and translate knowledge with emphasis on science writing and targeted communication. The initiative to establish the Science and Technology Branch has resulted in improved communication and understanding among researchers and the broader policy and program stakeholders (Karl Schaefer, Head - Strategic Science-Policy, Environment Canada, March 25, 2009, Toronto, Canada).

While disseminating information and developing communication materials are important in conveying scientific information, personal interaction among scientists and policymakers is also central to bridging the science-policy gap. The most common forum for information exchange and networking is conferences and workshops. According to Fraser, Gaydos, Karlsen 
and Rylko (2006), conferences are an opportunity for scientists and decision-makers from a wide range of disciplines to share knowledge and information. A benefit of these events is the ability to draw participants from not only the government sector but also external stakeholders. This could provide an opportunity for indigenous knowledge to be disseminated to governments that would otherwise be excluded. Conferences are also a venue for scientists and policymakers to discuss opportunities for translating science into policy and educational tools.

In 2001, the Canadian Council of Ministers of the Environment (CCME) directed Environment Canada to organize a workshop as a mechanism to strengthen the science-policy linkage between water managers and scientists across all federal and provincial governments. The intent of the workshop was to better inform the decision-making process by bringing science to the policy and program community; identify research needs and priorities; and to identify mechanisms for sustaining dialogue (Schaefer \& Bielak, 2006). Workshop reports were prepared and posted that captured presentations, synthesized research and policy and program needs and identified options for sustained dialogue. Organizers conducted a post workshop survey to assess the extent that the workshop was able to meet its intended goals. The majority of respondents indicated the event was a successful mechanism at linking research and policy (Schaefer \& Bielak, 2006). Specifically, policy and program managers indicated the workshop and products directly informed decision-making and scientists were able to identify research issues and needs.

Although opening communication channels between researchers and policy managers can lead to increased uptake of science in the policy process, there remains the challenge of sustaining these activities. Further, increased interaction between the communities does not necessarily lead to greater utilization of information unless mechanisms are put in place to allow 
policymakers to give feedback to scientists about their concerns and needs. The post CCME workshop survey also canvassed participants on ideas to sustain dialogue and improve networking. Participants indicated that a combination of electronic networking and face-to-face meetings are the main vehicles to sustain dialogue. There was preference to stay networked through some form of regular electronic contact, with occasional face-to-face meetings as the science evolves (Schaefer \& Bielak, 2006). These less formalized interactions could lead to healthier and stronger working relationships, allowing for information to flow more freely between the communities. These incremental steps could contribute to the paradigm shift desired to strengthen the science-policy interface.

\section{Stakeholder Engagement}

Engaging stakeholders outside the government realm can also help strengthen the science-policy connection. Environmental non-government organizations (ENGO) for example, can communicate research results to other stakeholders and the media, and in the process turn information into common knowledge (Ken Ogilvie, formerly Executive Director, Pollution Probe, March 27, 2009, Toronto, Canada). These ENGOs can also garner public support through education, particularly in cases where science and policy attitudes differ (Anderson, 2007). Adopting mechanisms and tools to enhance stakeholder involvement has the potential to narrow the divergence among scientists and policymakers by facilitating information exchange and uptake. Stakeholder involvement has the additional benefit of creating opportunities for integrating traditional or indigenous knowledge into the policy process that may otherwise be unintentionally overlooked.

The beginnings of a shift in knowledge production are also being observed. The traditional academe and government driven research continues, however other organizations 
such as think tanks and NGOs are also becoming prominent producers of knowledge. For example, the Canadian ENGO Pollution Probe not only has very strong links to the scientific community, it also seeks opportunities to engage in the science. "Pollution Probe is very proactive in terms of trying to get research dollars channeled toward scientific questions that are relevant to policy" (Ken Ogilvie, formerly Executive Director, Pollution Probe, March 27, 2009, Toronto, Canada). The practical science results produced by Pollution Probe undergo a process similar to a peer review whereby the scientific community and policymakers review the results. This information is then disseminated to policymakers to help inform policy development. A Great Lakes policy expert shared that funding is provided to some ENGOs to conduct research for this purpose (Interview, April 24, 2009, Toronto, Canada).

Stakeholders can also influence policy direction through formal channels such as advisory committees to governments. One such example is the Council of Science and Technology Advisors (CSTA) established by the Canadian federal government. Its membership is drawn from the academic, private and not-for-profit sectors, and reflects the diversity of science and technology-based disciplines. The CSTA was created in 1998, in response to the 1996 federal science and technology strategy, Science and Technology for the New Century. The CSTA provides advice to the federal cabinet on the strategic management of federal science and technology by examining issues that cut across science-based departments and agencies and highlighting opportunities for synergy and joint action (Industry Canada, 2002).

The utility of engaging stakeholders in the policy process will be further explored in the discussion on transboundary water management. Public participation in water management has become a critical component in policy development and as a way of strengthening the sciencepolicy connection. 
The desire to produce evidence-based policies is high among scientists and policymakers. "Without science, you cannot convince people of anything" (Interview, May 28, 2009, Kampala, Uganda). Although it is recognized that science does not provide all the answers, particularly as considerations of socio-economic and political implications are also part of the policy equation, it is essential that science informs the policy process to enhance its credibility. Failing to do this may lead to policy decisions that run counter to science and result in disastrous consequences (Interview, March 27, 2009, Toronto, Canada).

A central focus in this study is the examination of the science-policy interface in transboundary water areas. The complexities inherent to multilateral natural resource management have the potential to exacerbate the gaps characteristic of environmental policy development.

\subsection{Science and Policy in Transboundary Water Management: An Introduction}

Previously identified causes for the science-policy gap - the lack of understanding of science and policy cultures, processes and objectives; existing perceptions and paradigms; the politicization of science; and ineffective communication - are also observed in the transboundary water areas and in some instances are aggravated due to conflicts between riparian states. This suggests that the role of science and consequently the science-policy connection in environmental policy processes is a function of the context in which is it integrated.

In transboundary areas, agendas can be dominated by political views and conflicts, which prevent the 'mundane issues of science' from getting on the policy agenda (Interview, March 25, 2009 , Toronto, Canada). When the policy process is dominated by politics, science is rarely engaged and is only integrated when advancing political agendas. In these situations, the risk of science becoming politicized is high, thus widening the gap between science and policy. For 
example, one Ugandan policy expert shared an experience whereby one country reported different statistics for the same issue to suit different purposes. A statistical figure was reported to request support from a donor, which was different than the figure reported to defend a position. The interviewee indicated, "you can have different types of statistical information [for the same issue]...[reported by] the same country" (Interview, May 21, 2009, Kampala, Uganda). Politicization of science has also been observed in technical committees. Although there is an expectation that technical advisory committees provide objective advice for the management of transboundary waters, it was noted by a policy expert in Uganda that committee members also enter into discussions with the intent to advance their country's agenda. Scientific integrity under these circumstances can therefore be compromised.

Data conflicts can also influence the science-policy interface in international basins (Centre for River Basin Administration, 2005, p. 12). Harmonization of management policies requires monitoring methods to be standardized in order to have comparable monitoring data across jurisdictions. Scientists need to come to a consensus on the type of monitoring instruments used in the area and to ensure the same calibrations are made, otherwise there will be variations in the data, leading to incomparable information: "You cannot compare a goat with a cow" (Interview, May 21, 2009, Kampala, Uganda). The divergence between science and policy in these situations is of national importance as it has the potential to widen the gap between states. Inconsistent scientific information can delay collaborative efforts to effectively manage the common resource. As an example, Lake Victoria managers are grappling with issues of fish stocks. An interviewee previously involved with Lake Victoria management experienced a situation where two research studies revealed opposing results with respect to the state of the fish stocks (Interview, May 29, 2009, Kampala, Uganda). The policymaker was then left with the 
dilemma of whether or not action should be necessitated owing to conflicting information. This example illustrates the confusion that can arise from inconsistent information and the consequent inaction or delay in acting as a result of scientific uncertainty.

In the transboundary context, the science-policy interface can be further complicated by factors that are traditionally external to national policy processes. The aforementioned examples offer a glimpse into the intricacies of transboundary management regimes and the need to strengthen the science-policy connection in order to minimize the challenges indicative to transboundary water policy development. In the following chapters, this paper discusses the various components of transboundary water management regimes and explores tools and mechanisms that could bridge the science-policy gap in these areas. 


\section{Chapter 3: Transboundary Water Management}

\section{Introduction}

There are over 260 transboundary or international freshwater bodies that border two or more riparian countries. These resources sustain human and ecological existence within the lake and river basins and in some cases life downstream. As such, they are under tremendous pressure as a result of increasing and competing demands (Varis et al., 2008, p. 3). Emerging issues such as climate change will exacerbate these pressures. Attention to the management of these lakes is needed to alleviate existing challenges and to prevent conflicts that may arise between riparian countries. Investments in effective transboundary water management strategies can lead to the sustainability of the resource for future generations.

In the world of transboundary water management, the science-policy dynamic has the potential to become more complex and problematic. This could be attributed to differing approaches to natural resource management and the mere fact that there are inevitably more players in the process. The central position of this chapter is to demonstrate the utility of science in transboundary water management systems, in terms of existing approaches and the components required for effective management regimes. This discussion will provide the basis for the next chapter where the science-policy interface in the transboundary context is examined in detail.

This chapter begins by highlighting the challenges affecting shared water resources and by discussing the management hurdles confronting these areas. It explores the evolution of management approaches including the ecosystem approach, the highly promoted integrated water resources management approach, and the growing interest in adaptive management. The key stakeholders in the management of transboundary waters and their role in defining the 
science-policy interface are then discussed. This is followed by a discussion of the criteria that is deemed necessary for the design and sustainability of effective transboundary water management regimes. Lastly, as East Africa's Lake Victoria is a central focus of this paper, some comparative context on the African and North American Great Lakes is provided.

\subsection{Water Under Pressure}

The Global Environment Facility $(2009$, p. 3) estimates there are approximately 300 areas of potential conflict over water sharing. This is expected to intensify in the future, as two-thirds of the world's projected population of 8 billion will live in countries that suffer severe or moderate water stress. While there is international recognition that sustainable management of these resources is critical for human and ecological integrity, the deterioration of transboundary waters persists.

Waterways have traditionally been the dumping grounds for excess waste and byproducts. This has resulted in residual effects lasting long after activities cease. Ecological consequences include nutrient eutrophication and changes in biodiversity and ecosystem function. For example, discharges of phosphorus from sewage treatments plants into the Great Lakes led to increased nutrient loading and eventual eutrophication of Lake Erie in the 1960s (Sproule-Jones, 2002; Kayombo \& Jorgensen, 2006; Varis et al., 2008). Atmospheric deposition of sulphates and nitrates has resulted in approximately 14,000 acidified lakes in Canada (USEPA, 2010).

Even as point sources of pollution remain significant contributors to lake quality deterioration, non-point sources are becoming critical and potentially more difficult to manage. A Great Lakes scientist explained that poor agricultural practices and intensified urban sprawl are becoming significant sources of pollution that affect the quality of the Great Lakes 
(Interview, April 8, 2009, Windsor, Canada). However, managing non-point sources is challenging and is complicated by the scale and complexity of the problem, and by the issue of property rights (Johns, 2008, p. 205). Governments have traditionally "tended to limit the scope and focus of pollution management to more easily identifiable point sources" (Johns, 2008, p. 207). Managing these non-point sources requires a new mechanism to address this emerging issue. There are also variables with unknown yet inevitable adverse effects including those arising from climate change.

In transboundary water areas, the effects of anthropogenic activities could be intensified due to aggregated demands placed on the resource by riparian states. For example, water levels could be dramatically reduced to meet the demands of anthropogenic activities such as irrigation and energy production in all bordering nations. Water diversion and withdrawal are particularly sensitive issues as actions undertaken by one nation can affect the national security of another. As one policy expert noted, the biggest challenge in transboundary water areas is water security (Interview, May 21, 2009, Kampala, Uganda).

Population growth in transboundary water areas is also expected to increase dramatically in the future. Lake Victoria currently supports approximately 30 million individuals in the basin and this is projected to double by 2020 (LVFO, 2009; Awange \& Ong'ang'a, 2006). The increase in population will inevitably add to existing pressures facing the lake. Along with population growth is the development of national economies, leading to increased consumption and diversion of waterways. Transboundary areas are typically more prone to conflict as a result of competing interests (Varis et al., 2008; GEF, 2009).

There are numerous challenges confronting the management of transboundary waters. Adverse effects resulting from increased population and economic growth will lead to further 
deterioration of water quality and quantity. It is therefore critical to mobilize governments and international partners to strengthen management regimes in these areas to ensure the sustainability of these resources and to avoid future conflicts among riparian countries. Effective transboundary water management can enhance the relationships between nations and lead to what Grey and Sadoff (2002) describe as 'catalytic' benefits; those that extend beyond ecological benefits and include new or enhanced trade negotiations and opportunities to improve food and energy production, and food and drought management.

In response to the pressures noted, various approaches have been developed in an attempt to sustainably manage shared water resources. Management approaches have evolved in parallel with the growing scientific literature on the linkages within the ecosystem and the revelations of synergies and discordances associated with existing policies across sectors and anthropogenic activities. As such, management regimes have moved away from the traditional approach of managing commodity production; that is, managing resources independently without consideration for other environmental factors, to a more holistic ecosystem approach. As the science has continued to evolve, water resource managers are now adopting an integrated water resources management (IWRM) approach that considers not only linkages within the natural ecosystem but also socio-economic implications and the impacts resulting from cross-sectoral activities. Recently, the concept of adaptive management has been invoked more frequently. This paper will briefly discuss the concepts of ecosystem, IWRM and adaptive management frameworks to illustrate the role of science in the various management approaches that exist in transboundary water management regimes today. 


\subsection{Approaches to Managing Transboundary Waters}

Managing water resources in a holistic fashion recognizes the linkages between the water system and the ecosystem as a whole. In most transboundary water areas, managers and scientists have adopted an ecosystem approach to managing the shared resource. Management regimes are presently shifting toward an integrated approach to water management, taking into consideration the ecological and cross-sectoral linkages in the basin. Given that science is the basis for these water management approaches, water managers are beginning to appreciate the need to adapt to evolving science. Thus, there is growing interest in the adaptive management approach.

\section{Ecosystem Management Approach}

The ecosystem concept originated as strictly scientific (Bocking, 2006, p. 98). It holds that there are inter-related components in our environment and that decisions made in one area can affect another (Ontario Ministry of the Environment, 1993, p. 3). However, Bocking (2006, p. 96-97) suggests that there is no consensus on what ecosystem management is or what it implies. He offers three forms that ecosystem management could take, including:

1) A single focus on the production of a specific commodity; the understanding of the effects of all factors within an ecosystem on a particular species of interest;

2) A broader approach that emphasizes responsiveness to a number of human priorities, that not only encompasses commodity production, but also other services such as recreation and wilderness protection; and

3) One that is explicitly defined in terms of science and focuses on the state of the ecosystem itself. Commodity production or supply of other amenities is adjusted to be consistent with the preferred state of the ecosystem.

The first two scenarios are observed in existing transboundary water management regimes. For example, the management of Lake Victoria has adopted an ecosystem approach that focuses on the production of a specific commodity such as fisheries. In the North American Great Lakes management regime, the approach encompasses other human 'leisure' activities 
such as recreational swimming and fishing (United Nations University, 2008). While the ecosystem approach may be applied in different forms, scientific evidence is the foundation upon which policies and programs are developed.

As the scientific understanding of water ecosystems has evolved, revealing linkages between natural resource management and socio-economic wellbeing, water management regimes are shifting toward integrated water resources management (IWRM). Some water experts believe the ecosystem approach to water management complements the current thinking on IWRM (IUCN, 2009).

\section{Integrated Water Resources Management (IWRM)}

The integrated water resources management approach is becoming accepted as the way forward for efficient and sustainable development and management of the world's limited resources and for coping with conflicting demands (UN Water, 2009). With an increased understanding of the interconnectedness of ecosystem components and anthropogenic activities, IWRM builds on the ecosystem approach to address these linkages. It continues to maintain scientific integrity in the design of management policies. IWRM embraces a broader spectrum of management activities in order to maximize socio-economic benefits in an equitable manner without compromising the sustainability of vital ecosystems (Conca, 2006, p. 125). This approach is particularly important as socio-economic dependencies on depleting natural resources are becoming more apparent. The three interrelated themes identified in IWRM are (Conca, 2006, p. 124):

(1) Recognition of the full range of social, economic and ecological uses of water;

(2) 'Cross-sectoral' water management, in the sense of integrating planning and practices related to agricultural, industrial, municipal and ecosystematic or in-stream demands for water; and

(3) Water management at multiple scales and levels, in the sense of coordinating local, regional, national and transnational practices and institutions. 
IWRM requires strong integration of science in policy decisions as it attempts to incorporate the broad range of activities at all levels and sectors. This requires a sound understanding of the interrelated connections; science can help achieve this understanding. A key component to IWRM is acquiring better data and information to improve governance and integrated management. In principle, this approach has the added benefits of greater transparency, accountability and stakeholder involvement, all of which are consistent with the proposed mechanisms to narrow the science-policy gap as previously discussed.

Ecosystem and integrated water resources management include a degree of uncertainty as a consequence of being framed in scientific terms. As such, there is a risk of producing flawed policies if the uncertainties are not considered or addressed properly. In situations where knowledge of the underlying system processes is limited and a high degree of uncertainty exists, adaptive management provides guidance on the means to achieve the overarching goal of sound natural resources management; that is, to attain equitable, efficient and sustainable use of management resources (Centre for River Basin Administration, 2005, p. 29). While IWRM is becoming the dominant approach to water management worldwide, the concept of adaptive management has recently been invoked more frequently. Adaptive management addresses the uncertainty inherent in the production of scientific information and allows policies to adapt to evolving science.

\section{Adaptive Management}

Adaptive management is a proactive approach that attempts to incorporate flexibility into the design of water management frameworks to achieve the most effective and efficient means of managing natural resources. It implies a systematic process for continually improving management policies and practices by learning from the outcomes of operational programs and 
by adjusting practices based on what was learned (B.C Ministry of Forest \& Range, 2010; Bormann et al., 1999). Given this, by default, adaptive management incorporates an evaluation component into the policy process. This is essential to allow resource managers to fine-tune objectives and measures to changing circumstances as necessary.

Adaptive management builds on the principles of IWRM that emphasize transparency, accessibility, accountability, and stakeholder participation. It is a flexible system that operates across multiple levels of government, industry and various non-government organizations representing a broad spectrum of interested groups. It also stresses cross-sectoral analyses to address the various demands on the water resource. This implies a closer relationship among scientists, managers and resource users and the need for all parties to participate in the planning and implementation of research (Bocking, 2006, p. 93). Armitage and Doubleday (2007, p. 286) observed that successful implementation of adaptive management depends upon the development of trust and shared understanding of the systems among the people involved in management - a crucial component to narrowing the gap in science and policy. Yet, of course, this is also an extremely difficult goal to achieve amongst states that are competing over the use of a water body.

Notwithstanding the potential benefits of this approach, the magnitude of the challenges and complexity associated with implementation make adaptive management prone to resistance. The approach can be both timely and costly, and its results can also be paradoxically ambiguous (Bocking, 2006, p 94). When asked to comment on the adaptive management approach, policy experts noted that while the concept is sensible in its intent, the application of adaptive management is challenging. The current policy infrastructure established for managing resources cannot respond to new information quick enough to effectively adapt (Interview, April 
17, 2009, Toronto, Ontario). Further, it has been observed that adaptive management is only effective in simpler political contexts where there are relatively few interest groups and a single resource agency that has a clear jurisdictional mandate (Bocking, 2006, p. 95), and where there is sufficient scientific and political will (Interview, April 30, 2009, Toronto, Canada). Complex institutional circumstances such as those in transboundary water areas may not be conducive for the adoption of adaptive management.

The ecosystem, IWRM and adaptive management approaches all have science as their fundamental building block. As these management frameworks are instituted in transboundary water management regimes, one can posit that strengthening the science and policy connection is a necessary requirement to ensure management systems are designed effectively. Achieving optimal outcomes therefore requires that the major players in transboundary areas work together to bridge the science-policy gap. Key players in transboundary water management are identified in the next section and the degree of their influence on the integration of science in the policy process is assessed.

\subsection{Major Actors in Collaborative Water Management}

The management of transboundary waters involves a multitude of actors. The nature and quantity of participants are dynamic and dependent on the specific requirements of the resource. These actors and the extent that they are involved in the design of management policies could influence the role of science in the policy process because of their capacity to produce or receive scientific information. There are four broad categories of individuals or groups of individuals that have key roles in transboundary water management regimes.

Transboundary water management systems would not be realized without the involvement of national and sub-national governments. However, central state actors are not the 
only ones involved in transboundary water management (Timmerman \& Langaas, 2005, p. 181). Commissions or organizations that have as their main function to coordinate activities within the basin are common in most transboundary areas. Non-government participants are also required to ensure management policies address local and community concerns and help facilitate policy uptake and implementation. Lastly, in areas where resources are limited, participation by international partners such as the World Bank and Global Environment Facility (GEF) or other multilateral agencies are critical in the design and sustainability of transboundary water management regimes.

\section{National and Sub-national Governments}

The management of international waters requires strong commitment and participation by national, sub-national and local governments. All three tiers of government create an enabling environment through the establishment of policies and legislation that constitute the 'rules of the game' (GWP, 2009). The government can also develop implementation frameworks and enforcement provisions to ensure management policies and programs are put in practice and policy objectives are achieved.

A national government is the custodian of the nation's water resource (Asmal, 1998, p. 98). For the reason that transboundary waters cross lines of sovereignty, activities in these areas usually fall under the mandate of federal or national governments. National involvement is particularly critical in the beginnings of management design, where the initiation of interjurisdictional dialogue is made and communication channels are opened. In preparation for these negotiations, national governments typically require scientific knowledge on the state of the lake or river ecosystem. As such, scientific capacity is primarily developed at the national level as investments in research are critical to ensure all riparian states have the most accurate 
information. For example, the Directorate of Water Resources Management of Uganda's Ministry of Water and the Environment has, among its various functions, the responsibility of monitoring water quality and quantity. This monitoring data can inform the national government of trends and reveal emerging issues in Lake Victoria.

Sub-national governments have the role of advocating their interests in the development of transboundary water management policies. They are also the primary implementation partners for commitments made under bi- or multilateral agreements. The Province of Ontario plays a strong implementation role for commitments made under the Great Lakes Water Quality Agreement and the Canada-Ontario Agreement (COA) Respecting the Great Lakes Ecosystem. In some cases, Ontario also directly negotiated transboundary agreements with sisterjurisdictions in the United States to address regional and sub-national concerns (Interview, Ontario Ministry of the Environment, April 24, 2009, Toronto, Canada). Sub-national governments also have a research function and can enhance monitoring capacity for their region.

Local or municipal governments assist with the implementation of transboundary policies and programs and work with sub-national governments to voice localized interests to national governments. As local governments are much closer to citizens, they also undertake important outreach and education activities. Effectively communicating lake management objectives to the community can facilitate policy uptake and implementation, both of which are critical to the success of transboundary water management.

\section{Transboundary Commissions and Organizations}

Common to most transboundary water areas is a structural administrative or coordinating body, usually in the form of a commission or other governing organization (Centre for River Basin Administration, 2005, p. 4). Commissions are critical to the overall governance of 
transboundary waters and can alleviate issues resulting from fragmented policies and programs. They can also be requested to identify needed laws and harmonize and prioritize research areas to help direct national investments and coordinate collaborative research studies (Borre, Barker \& Duker, 2001, p. 205).

These entities can vary in type and purpose according to the political and cultural context and the water resource challenges in the area (GWP, 2009). As an example, the Lake Victoria Fisheries Organization (LVFO) was established in 1994 to address fisheries issues within the basin. With increased understanding of the lake ecosystem, the Lake Victoria Basin Commission (LVBC) was subsequently created in 2004 upon ratification of the 'Protocol for the Sustainable Development of the Lake Victoria Basin' to govern a broader suite of lake activities. The LVFO and LVBC have prescribed functions to coordinate activities between the two entities and with national governments. The three riparian countries are represented in the LVFO and all five basin countries in the LVBC (Kenya, Uganda, Tanzania, Rwanda and Burundi). Members have equal opportunity to express their views and concerns, and contribute to the design of management policies and programs. The strength of commissions and governing organizations is the support and commitment by all national governments.

While transboundary coordinating organizations are essential for management actions across political boundaries, there are limitations to their reach. The Centre for River Basin Administration (2005, p. 26) notes that commissions often have a technical bias, are limited in size and have an abstract level of thinking, which often hinders the involvement of stakeholders and the public. The sustainability of the commission is also dependent on a secure funding base, the political will of governments and the commitments of the partners who create them (GWP, 
2009). In areas where resources are limited and political instability is high, the risk of commissions failing as a result of these external influences is also elevated.

\section{Non-Government Participants}

It was not too long ago when management policies were developed within the walls of government, with little or no consultation with external stakeholders. Recent trends for increased participatory approaches in transboundary water management suggest that nongovernment participants are becoming progressively more important players in the policy domain. A policy expert interviewed for this study highlighted that the first step in IWRM is the creation of a multi-stakeholder group comprising of members from government, civil society, research institutions and donors (Interview, June 11, 2009, Entebbe, Uganda). As policies are formulated and new initiatives are proposed, policymakers are made aware of concerns and potential adverse impacts on the community through this multi-stakeholder group. Governments are recognizing that policy success in both design and implementation can hinge on nongovernment input, support and uptake. Stakeholders have the ability to build trust between project processes and civil society, which leads to transparency of decisions, informed decisionmaking and cost effective solutions (National Consultative Meeting between Uganda CSOs and LVBC Proceedings, 2009).

As introduced in Chapter Two, non-government participants can help bridge the sciencepolicy gap by translating and communicating technical information to a broader public audience. Instituting a mechanism for public participation can also encourage the uptake of indigenous knowledge to inform the policy process. The importance of non-government participation as a criterion for the development of effective transboundary water management regimes will be 
observed later in the chapter. This will be further elaborated in the case study on Lake Victoria where the participatory approach has been instituted in the fisheries management system.

\section{International Partners}

In areas of the developing world where there is a modicum of resources, international partners can be significant players in the development of transboundary water management regimes. This is particularly evident in areas where management systems are only incipient, and national commitment, resources and capacity are low. In these situations, international players such as the World Bank and Global Environment Facility can initiate the actions and leverage the resources necessary to convene riparian countries in discussions on collaborative management.

Seeing as these international partners have the power of the purse, they can have considerable influence over the outcome of management policies and programs even though their functional role is limited. The Centre for River Basin Administration (2005, p. 59) suggests international partners should be aware of their influence on management outcomes and to ensure that resources are dedicated to management activities of highest priority to the recipient. Further, international partners can inadvertently contribute to monitoring gaps in developing countries as a result of project cycles. These countries typically do not have the ability to maintain monitoring activities post project completion due to fiscal constraints. Notwithstanding these challenges, donors can advance transboundary water management activities by: supporting the conclusion of international treaties; supporting the resolution of underlying issues and promoting action on the ground; supporting social and economic change in the basin; and providing

continuing support after the conclusion of the agreement (The Centre for River Basin Administration, 2005, p. 24). 
The Global Environment Facility (GEF) for example, is a global partnership among countries, international institutions, NGOs, and the private sector to address global environmental issues while supporting national sustainable development initiatives. It provides grants for projects related to six main areas including international waters. In these activities, GEF plays a catalytic role in helping nations make full use of policy, legal and institutional reforms and investments necessary to address complex concerns about transboundary water management (GEF, 2005, p. 1). Through its programming, the GEF has helped 172 countries work together on shared water systems by leveraging $\$ 1.1$ billion in GEF grants and $\$ 4$ billion in co-financing (GEF, 2009, p. v). The GEF was a key player in the design of the Lake Victoria Environmental Management Program (LVEMP), which strived to improve scientific capacity within the basin. The LVEMP will be discussed later in the case study on Lake Victoria.

As one can observe, the multitude of players creates a complex environment for managing transboundary waters. The extent of their involvement can influence the degree that science is integrated into management frameworks and policy processes. It is therefore important to recognize that strengthening the science-policy interface in transboundary water management regimes does not rest solely on scientists and policymakers but requires a collaborative effort among the various players in transboundary water management. While this creates a very challenging set of circumstances in principle, effective collaboration will help establish the criteria necessary for the design of effective regimes as will be discussed in the following section.

\subsection{Criteria for Effective Transboundary Water Management}

In designing a transboundary water management system, policymakers must strike a fine balance among socio-economic, political and environmental priorities. How do riparian states develop a 
regime to manage transboundary waters sustainably and efficiently while still using the waters for economic and social gain? Can such regimes produce a win-win situation for all parties while maintaining the integrity of the water resource?

The literature and interviews suggest some common criteria for effective transboundary water management. Effective regimes can be defined as the "degree to which... agreements and organizations lead to changes in behaviour that help solve collective problems" (Finger, Tamiotti \& Allouche, 2006, p. 15). First, strong national leadership and commitment by all countries in the basin is critical for initiating and sustaining the dialogue necessary for joint management. This is supported by the need for mutual recognition of shared responsibility and accountability over the resource - a second criterion for collaborative management. Mutual recognition of responsibility typically leads to the third criterion: the signing of a cooperative agreement that codifies the commitment by partner states. These agreements commonly establish the creation of a transboundary coordinating mechanism that governs all basin activity. This is also deemed to be a necessary component for effective transboundary water management. Lastly, public participation and building scientific capacity to enhance the credibility of policies and key players are also common criteria for collaborative management. While these six criteria are not exhaustive and can differ in their application to developed and developing nations, they are a sound reflection of the main elements suggested as necessary for successful transboundary water management regimes. The role of science is interwoven throughout the discussion to illustrate its influence on achieving the criteria.

\section{Strong National Leadership and Commitment by All States}

Transboundary water governance requires national leadership and sustained commitment by all partners in the basin. While independent nations could be in a state of political readiness that 
impels them to take the lead, their success is dependent on the willingness of their neighbouring partners to participate in transboundary water negotiations. To increase the likelihood of participation by countries within the basin, it is necessary to forge strong relations by building mutual trust. This diminishes the influence of more powerful states and prevents potential conflicts by creating an enabling environment.

Building a good political atmosphere and maintaining good relations is the most powerful strategy for reaching consensus (Centre for River Basin Administration, 2005, p. 20). A number of interviewees stressed that trust is the most critical factor in international coordination and negotiations, albeit it is difficult to attain. As previously observed one way in which trust could be gained is through the sharing and exchanging of scientific information on the state of the water body at issue throughout the policy development cycle.

A key role the GEF plays in transboundary water management is enabling an environment where countries can begin to exchange information and initiate dialogue with the objective of building mutual trust. A policy expert in the Lake Victoria basin observed that building trust "seems to be a natural requirement in this type of [multilateral] cooperation... a lot of money has been spent, a lot of interaction, a lot of support...from the donor community was required to enable processes to move forward" (Interview, June 11, 2009, Entebbe, Uganda). However, it was also observed by this same individual that the Lake Victoria riparian states are still struggling with issues of trust.

Active support and long-term commitment by top-level political representatives are also key to ensuring initial efforts are not abandoned. It is not uncommon for political representatives to be engaged only in the beginning stages of establishing a transboundary water management strategy, with diminishing involvement as the process ensues and government officials take the 
place of leaders in negotiations. In 1977, Le Marquand identified national leadership commitment as a principal requirement for successful riparian cooperation (Bernauer, 2002, p.

3). This remains true today. In areas where national governments are less committed, it is more difficult to develop and implement joint management strategies (Centre for River Basin Administration, 2005). Therefore sustained leadership and commitment in transboundary water management activities is an important criterion for the development of transboundary water management systems.

\section{Recognition of Mutual Responsibility and Governance}

To support the criterion of national leadership and commitment, countries must be presented with information that allows them to recognize their shared responsibility for the sustainability of the resource. Scientists can provide this information to national governments. Science and policy experts, as well as political leaders all must agree on the scientific information, and scientific uncertainty for that matter, before mutual recognition of shared responsibility and governance is to occur. The mutual recognition of shared responsibility and governance is the second factor suggested for successful transboundary water management.

Cooperation can only take place when a common understanding of the situation is reached and reciprocal interests are recognized (Timmerman \& Langaas, 2005, p. 180). Countries need to understand the scope of benefits and challenges that could potentially result from collaboration as well as the consequences of continuing with independent management practices. Scientific research and other technical undertakings such as benefit-cost analyses can help policymakers define national responsibilities in transboundary water management regimes. Research results can also identify the priority issues affecting the water body and help define policy objectives and explore potential solutions. Researchers and policymakers must therefore 
work closely together and across administrative boundaries to delineate the parameters necessary for the production of the 'right' information. Preparation of such information, especially in transboundary water negotiations cannot be over-emphasized (Timmerman \& Langaas, 2005, p. 185).

Only when countries mutually recognize their roles in the management of the shared water body will national governments commit resources to transboundary activities. For example, through the Nile Basin Initiative (NBI), countries are now moving towards thinking of the Nile River as a transboundary resource that was previously absent (Interview, June 9, 2009, Kampala, Uganda). Although the NBI has yet to result in a cooperative agreement signed by all Nile basin countries, the initiative was successful in providing relevant information that illustrated the state of the river and role of riparian countries. Transboundary water management activities are increasingly being incorporated into some national plans and budgets (Interview, June 9, 2009, Kampala, Uganda).

Failing to recognize mutual responsibility over a shared resource perpetuates fragmented management practices that could result in adverse effects on neighbouring riparian countries. Lack of scientific evidence that demonstrates the utility of collaborative management can delay this recognition and coordinated action. There are also other situations where self-serving agendas prevent the recognition of mutual responsibility and governance over the transboundary water body. This is observed in the Mekong River Basin for example, where China and Myanmar are only dialogue partners in the management of the river and are not obliged to meet the commitments under the Agreement on the Cooperation for the Sustainable Development of the Mekong River Basin (Mekong River Commission, 2009). In both circumstances, a lack of 
recognition of shared responsibility and governance can potentially lead to unsustainable water management practices and exacerbate lake deterioration.

The mutual recognition of responsibility over shared resources is an important factor in transboundary water management. Understanding the benefits of collaboration and the issues facing the water body can help countries facilitate the development of joint management strategies. Mutual recognition of responsibility can be formalized in cooperative agreements.

\section{Cooperative Agreements}

A third criterion for the development of effective transboundary water management is the establishment of formal cooperative agreements between riparian countries. Formalizing mutual responsibility strengthens the partner states' intent and commitment for establishing a transboundary water management framework. It also acts as an accountability mechanism that prevents one country from undertaking actions that could potentially have adverse effects on the shared resource. The signing of an agreement reflects political commitment and leadership to the sustainable management of transboundary waters and is typically the first milestone for those seeking to establish joint management frameworks because it codifies the commitment.

In some cases, the extent that transboundary management activities are implemented is dependent on the signing of a formal collaborative agreement. A policy expert interviewed for this study noted that it is difficult to observe any progress before legislation for a transboundary water body is enacted or agreed upon at the Ministerial level (Interview, March 26, 2009, Toronto, Canada). An interviewee at the Nile Basin Initiative supported this observation. Over the past 6-7 years, through the efforts of the NBI, basin countries have been negotiating an agreement for the region. Until such time when consensus is reached for an agreement, a governance provision to establish a basin commission, as well as information exchange and 
monitoring protocols cannot be realized (Interview, June 11, 2009, Entebbe, Uganda). In situations such as this, political differences among the basin countries are the limiting factor for collaboration. Meanwhile most Nile countries continue to develop water use projects independently. Hence, the mutual benefits of cooperation must be seen to be more valuable than going alone. Conducting collaborative research or collective science can assist riparian states with recognizing these mutual benefits.

In some situations, transboundary agreements are also a prerequisite in securing international funds. Varis et al. (2008) states that lending institutions are hesitant to provide funding to countries for transboundary water development that are without a cooperative treaty. The Global Environment Facility (GEF) in particular, collaborates closely with environmental conventions and agreements. GEF projects are often associated with the establishment of regional conventions as a show of the partner states' commitment to sustainability after the conclusion of the project (GEF, 2005, p. 1).

With respect to the content of agreements, the literature identifies several elements that are common (Kliot, Schmueli \& Shamir, 2001; Centre for River Basin Administration, 2005):

- A duty to cooperate and negotiate in good faith with genuine intention of reaching consensus;

- Prohibition of management practices likely to cause substantial injury to other states;

- A duty of prior consultation;

- Principles of equitable utilization;

- Establishment of a transboundary commission or organization; and

- Protocols for information exchange.

It has also been suggested that transboundary agreements that have conditions for monitoring tend to have stronger implementation success (Ma, Hipel, De \& Cai, 2008, p. 1079). For example, the 1998 Convention on the Protection of the Rhine provides that the Commission has the power to establish international monitoring programs on Convention implementation. 
The monitoring results are used to measure the effectiveness of the decision implementation. Furthermore, each Contracting Party is requested to establish its own monitoring programs and analysis on the Rhine ecosystem and report the results to the Commission. The inclusion of these provisions in the Convention adds strength to monitoring activities and formalizes a role for science. Equally, data and information sharing protocols as well as technical secretariats facilitate better management (GWP, 2009).

While collaborative agreements may, on the surface, bind the countries to take action on transboundary water management activities, it was noted by several interviewees that implementation of the agreements can be challenging. As one respondent noted, "there are a lot of nice words in these agreements, but then how do you actually implement things like a procedure for sharing data information or procedure for water quality management, or a procedure for maintaining minimum flow" (Interview, June 11, 2009, Entebbe, Uganda)?

Among the various components included in these agreements, the literature identified the creation of a coordinating entity as the fourth key factor to effective transboundary water management. These commissions have the ability to convene all the critical players in the management of the water body and function to coordinate management activities including guiding and conducting collaborative research.

\section{Transboundary Commission or Organization}

Given the complexity of issues in transboundary water areas and the number of players involved with management activities, the creation of a regional body is deemed necessary to ensure appropriate actions are coordinated for the sustainability of the resource. As previously discussed, establishing a coordinating mechanism is often a provision in cooperative transboundary agreements. These mechanisms are usually in the form of a commission or other 
governing structure. Once established, commissions or coordinating bodies become major players in transboundary management regimes.

Commissions create a forum for collaboration and dialogue, allowing for information exchange and the development of joint management strategies. They can also act as a moderating factor within a conflict situation (GWP, 2009). All basin countries are represented in transboundary commissions and have equal opportunity to voice their concerns and contribute to the design of management activities. In some cases, these transboundary organizations have representation by Ministers and are granted authority to establish legally binding provisions. The Lake Victoria Fisheries Organization for example is led by a Council of Ministers who can introduce new policy to parliament to be passed in their respective countries (Interview, May 26, 2009, Jinja, Uganda). Policy decisions are to be made by consensus by all three riparian states. In other areas, commissions have an advisory function with limited sovereign authority. For example, "[m]uch of the debate about the effectiveness of the IJC...has concerned the fact that the binational agency has no powers to enforce its recommendations (Botts \& Muldoon, 2005, p. 20). Finger et al. (2006, p. 16) further observed that that the IJC has the power to investigate pollution problems but does not have the power to implement them.

This paper previously indicated that transboundary organizations also have the function to harmonize management activities and efforts across all partner states. This includes harmonizing standards and data collection within the basin and reaching consensus on priority issues facing the water body and areas for collaborative research. Achieving harmonization is a means of forging stronger relations among the various nations, and is necessary for sustained leadership and commitment. At the same time, the challenges associated with harmonization, standardization and ultimately the integration of scientific evidence in transboundary 
management plans escalates tremendously in comparison to a national setting given the complexity of the issues and range of actors and power dynamics. Further, included in the mandate of most transboundary organizations is an expectation or obligation to engage public stakeholders in the policy process. Given the array of responsibilities bestowed on transboundary organizations, they are a necessary component in the design of effective transboundary water management systems.

\section{Public Participation}

Public participation is the fifth factor deemed necessary for successful transboundary water management. Building in mechanisms to enhance stakeholder involvement is critical to the overall success of the management system. In doing so, it is expected that stakeholder concerns and unforeseen challenges can be identified and addressed early in the policy process. This also has the potential to facilitate policy uptake and implementation. A stakeholder or the 'public' in this context is taken to mean a person or group with a direct interest or/and involvement in the sustainability and management of the water body.

As noted in the previous chapter, a strong public participatory approach can help build trust among the community and governments. NGOs can translate technical information and policy goals to the broader public, facilitating understanding and implementation. A strong presence of non-government actors also increases transparency and accountability of government decisions. Commissions can play a role in strengthening public participation by creating a mechanism for their involvement in the policy process. The International Joint Commission for example, organizes a biennial public meeting that draws in hundreds of concerned stakeholders as a means for communicating stakeholder concerns to governments. 
Stakeholder engagement can also provide a means of introducing indigenous knowledge into the decision-making process and improving lake stewardship among the community. For example, a key component of the Lake Victoria management regime that will be explored in the case study is the creation of Beach Management Units (BMUs) at landing sites across all three riparian states. As fisher communities are the direct users and beneficiaries of the lake's resources, they have practical knowledge on lake dynamics that may not be captured by traditional scientific research. Further, their daily interaction with the lake can offer critical information on anomalies observed on the lake. Instituting BMUs into the LVFO has created a mechanism that allows fisher communities to inform lake managers and scientists about lake characteristics that would otherwise be absent. In principle, they do so through government fisheries officers who are represented at each of the landing sites, who then convey this knowledge and local concerns to policymakers (Interview, May 26, 2009, Jinja, Uganda). This mechanism also has the added benefits of instilling a sense of ownership, responsibility and accountability for lake resources. Recognition and support of fishers is one way to ensure promotion of sustainable utilization of lake resources as well as conservation of the environment (Kayombo \& Jorgensen, 2006).

The advantages of public participation do vary however, and are dependent on the political climate of the state. In some developing countries, public participation has very little influence on water management and is only carried out to meet the criteria necessary to acquire international funding. For example, one ENGO respondent shared that in the case of "the [Uganda] Bujagali hydroelectric project...the company that was promoting the project...hired all these trucks to go into the village and bring people into the public hearing. The World Bank can go and write in the case of this project, we conducted a public hearing" (Interview, May 21, 
2009, Kampala, Uganda). In other cases, public participation can in fact hinder progress. "There has been a proliferation of civil society and non-governmental organizations that, however wellmeaning, are often non-accountable and may operate from a narrow self interest with no responsibility for the consequences of their actions" (GWP, 2009). A National Environment Management Authority (NEMA) staff member echoed this concern: "one has to be careful because we also realize we have some NGOs whose interest...may be to please their masters who fund them..." (Interview, June 1, 2009, Kampala, Uganda).

Notwithstanding these observations, strong public participation is considered important to the success of transboundary water management and can be a means of narrowing the science and policy divergence by building trust among stakeholders. While there have been examples where public participation has assisted with policy uptake and implementation, and building scientific capacity through monitoring and data collection, stakeholder engagement also has the potential to delay action in transboundary water management. It is therefore essential to define and communicate the role and expectations for non-government participants to ensure benefits are maximized from their involvement.

\section{Enhanced Credibility through Enhanced Science}

A final factor deemed necessary for transboundary water management is for key actors, policy processes and information to hold credibility. When asked about the role of science in the development of environmental policies, the majority of interviewees agreed that the application of science in the policy process adds to the integrity of the process and enhances the credibility of policymakers and policy outcomes. As one interviewee stated, "policies are opposed if they are not based on scientific facts. Generally there is no opposition if [there is] scientific evidence to support the policy" (Interview, May 28, 2009, Kampala, Uganda). Establishing credibility in 
the system and its players by integrating science into policies and management can help facilitate the building of mutual trust necessary for national leadership and commitment in transboundary areas.

It was previously observed that the quality of relations between countries could determine the extent of cooperation in transboundary management and consequently influence policy outcomes. Sound scientific research, along with the appropriate legal advice and economic information can be used to inform political decisions and policy objectives. This has the potential to lead to stronger partnerships and policies that ensure the sustainability of lake resources (Sadoff et al., 2002; Odada \& Olago, 2006). This requires political leaders and policymakers to have accessible and comprehensible information to make functional, logical and rational decisions (Timmerman \& Langaas, 2005, p. 178). This observation reinforces the need to effectively translate scientific information into understandable formats. This however remains a challenge in transboundary areas, and could be exacerbated as a result of cultural and language differences.

The recognition that science can enhance credibility of management regimes and its players is also reflected by the inclusion of scientific provisions in cooperative agreements and in mandates of transboundary commissions and organizations. As previously noted, cooperative agreements typically include provisions for harmonizing standards and research efforts and for exchanging technical information between countries. Some interviewees suggested that the harmonization of data and standards of measurements and methods across administrative boundaries is the most important function of transboundary commissions. Additionally, as transboundary management regimes are moving toward an integrated water resources 
management approach, operational support and technical cooperation on information collection and knowledge exchange are pre-requisites (Lorenz, Gilbert \& Cofino, 2001, p. 127).

In this chapter, one can observe that science directly and indirectly underpins current approaches to transboundary water management and is the underlying element in the criteria suggested for effective water management regimes. The key actors can influence the degree that science is integrated in the policy process and the direction that scientific research can take. Given that science is interwoven throughout the various facets of transboundary water management, I have argued that taking a positivist approach to managing transboundary waters is appropriate for strengthening the science-policy connection in this framework.

In the next chapter, the thesis focuses specifically on causes of the science-policy divergence in the context of transboundary water management and identifies some tools and mechanisms to strengthen this interface. I apply the observations of the previous two chapters in this discussion and discern the reasons for the science-policy disconnect generally, and how these are exacerbated in transboundary areas. Further, this paper draws some parallels between the instruments used to narrow the gap in transboundary settings to those identified in environmental policy development generally.

Prior to this discussion, it is important to provide some general context on the North American (Laurentian) and African Great Lakes. The management of the Laurentian Great Lakes is well established and internationally renowned as a model of success. Lake Victoria governance has undergone various transformations and has only recently adopted a more collaborative and holistic management regime. The similarities and differences in the approaches adopted by developed and developing countries are a result of topographical, ecological and political differences. 
The properties that distinguish African lakes from their North American counterparts include their age, sensitivity to climate change impacts, long residence times, persistent stratification, continuously warm temperatures at all depths, major ion composition and propensity for nitrogen limitation (Odada \& Olago, 2006, p. 433). Lake Victoria is similar to Lake Superior in that they both have large surface areas, making them more susceptible to atmospheric deposition. However, they differ in their depth and further, because African lakes are poorly flushed systems, even when compared to Lake Superior, nutrients are highly retained in these areas (Hecky, Bootsma \& Odada, 2006, p. 207).

Political and historical relations among African nations and those in North America are also vastly different. These differences can significantly influence the outcomes of transboundary water management policies as management regimes are designed to coordinate management activities based on hydrological boundaries with those that are based on administrative boundaries (Varis et al., 2008, p. 8). In areas where there exist multiple administrative boundaries, the risk of conflict is high as in the case of the Nile River. While it is outside the scope of this thesis to explore in detail the impacts of historical and political relations on transboundary water management, the issue of politics and its influence on the science-policy interface will be addressed in the following chapter.

Given these differences, lessons and management practices in one area cannot be directly applied to another without consideration for its respective uniqueness. There are lessons learned that could be used to inform the design of management policies and practices, including those that can forge stronger relations between the science and policy realms. 


\section{Chapter 4: The Science-Policy Connection in Transboundary Water Management}

\section{Introduction}

In transboundary water areas, policymakers must consider national administrative, cultural, institutional and policymaking contexts and conditions as all can influence policy implementation (Timmerman \& Langaas, 2005, p. 180). Notwithstanding these challenges, this thesis argues that forging stronger linkages between the science and policy realms can lead to more effective transboundary water management regimes. For example, science can reduce the uncertainty between states and create a safe environment for governments to begin deliberations on the state of the lake and explore collaborative solutions for effective management (Lorenz, Gilbert \& Vellinga, 2001; Centre for River Basin Administration, 2005; Timmerman \& Langaas, 2005). This is particularly important in transboundary areas where competing agendas and conflicting political views can divide riparian countries. While this paper argues that scientific integration in the policy process can influence policy direction and outcomes, it is also recognized that transboundary water policies could be influenced by other extenuating factors that are not controlled by scientists and policymakers as in cases of political interference and resource constraints.

In this chapter, I delve deeper into the science-policy dynamic and observe that the divergence has the potential to become exacerbated in transboundary water areas. This paper explores the reasons for this gap and identifies tools and mechanisms to draw the science and policy communities closer. These instruments can also help facilitate the achievement of the suggested criteria for the development of effective water management. It then provides a brief discussion on the North American Great Lakes to illustrate the science-policy connection in a 
management regime that is long standing and well established. The observations gained from these discussions will be applied in a case study of Lake Victoria in Chapter Five.

\subsection{The Science-Policy Disconnect in Transboundary Water Management}

While forging stronger linkages between science and policy may be desirable in areas of shared resources, there are factors that prevent this from coming to fruition. In transboundary water management regimes, the causes of the science-policy divergence go beyond disciplinary differences and extend to variations in national approaches, cultures and demands on the resource. Notwithstanding the unique challenges of transboundary systems, many of these mirror those of environmental policy processes generally, including the lack of understanding of science and policy cultures. The literature and interviews revealed that some of these causes could be aggravated, as illustrated previously, with issues of politicization and inconsistencies with scientific information. Communication challenges can also be exacerbated and will be discussed in this chapter. There is also an added complexity to managing international water bodies as the magnitude and number of issues is typically much larger than managing issues within single nations. This can lead to fragmented policies and research efforts between states, which has the potential to further divide science and policy communities as well as riparian countries. Further, there are reasons that are explicit to transboundary areas that tend to be inextricably linked to the political dynamics among states.

\section{Communication Challenges}

A key factor for bridging the science-policy gap is the ability to effectively communicate scientific and technical information to the users of that information. Chapter Two confirmed that communication challenges are inevitable when there is a lack of understanding of disciplinary 
cultures, objectives and processes among scientists and policymakers. While this is also evident in transboundary water areas, the degree of communication challenges could be intensified as a result of cultural and political differences between riparian states.

Transferring knowledge from one country to another must take into account specific cultural and political contexts (GWP, 2009). Failing to do so effectively could divide the science and policy realms. For that reason, a critical step in the transboundary water management process is to create a shared understanding about the state of the lake, priorities and potential solutions. Science has an important role in facilitating this shared understanding by painting a picture of the current state of the lake using the strokes of scientific and monitoring information to the extent that it is available. Scientists must collaborate across administrative lines in order to engender a common scientific understanding and to package this information in an understandable way for policy and decision-makers in all partner states. Achieving a shared understanding of the lake could minimize the risks associated with communication barriers arising from language and cultural differences, issues of confidentiality and incompatibility of data.

The mere fact that there are more players involved with the management of transboundary waters can disrupt communication channels between scientists and policymakers and between nations. Transboundary water managers attempt to develop harmonized policies and in doing so, require clear, concise and consistent information to inform policy development. Policymakers are also required to clearly communicate information to decision-makers in all bordering countries. This requires some effort on the part of scientists to package and disseminate the information in a way that is easily understood, explicit in its intent and consistent in its messaging at each stage of the policy process and in every country involved. However, as 
previously indicated, scientists generally do not possess the skills to effectively translate information to policymakers and/or they are hesitant to engage in a process that is deemed political or that may compromise their objectivity. These observations are also evident in transboundary water areas. Failing to assemble information in management-friendly and community-friendly packages that clearly convey the benefits of collaboration and investments could prevent initial and sustained action to be taken in the transboundary water area.

\section{Policy and Research Fragmentation}

In Chapter Two, the challenge of producing and reporting inconsistent information in transboundary water areas was highlighted. The inadequacy of information can lead policymakers to undertake "more speculative policymaking' (Interview, April 24, 2009, Toronto, Canada) increasing the risk of producing ineffective and potentially erroneous policies. But perhaps the greatest problem facing lake basins around the world is fragmented policies (Borre et al., 2001, p. 204) as a result of disjointed information production and reporting.

Policymakers and scientists interviewed for this study highlighted the lack of coordination and prioritization of research efforts as a common barrier to achieving a strong science-policy interface. Scientific research tends to be conducted within national boundaries and applied to the development of national policies that appropriately respond to national needs. This 'siloed' approach to management activities can lead to unanticipated tradeoffs and fails to recognize synergies and the full potential of existing policies: "Governments are...fragmented and lacking in holistic systems-analysis capabilities" (Dale, 2001, p. 113). Further, due to the numerous issues facing transboundary water management, policymakers in bordering countries must define and agree on management priorities in order to guide research investments. The prioritization of issues will help scientists in riparian countries produce policy-relevant research 
for the region, rather than conducting research within their own borders. As one interviewee noted, there are a multitude of activities happening within the ecosystem and it is impossible for scientists to conduct research into all those areas. In such cases, value judgments have to be made about where the science focus is going to be.

The degree that riparian countries can respond to management issues varies from country to country leading to knowledge imbalance and gaps. Resource limitations can influence the extent that adequate information is made available to policymakers. This tends to be more of an issue in poor countries where resources vary significantly between states. Developing nations are also more susceptible to the problem of fragmentation because of the uncoordinated influx of international assistance (Varis et al., 2008, p. 93). As several interviewees noted, the extent that monitoring data is collected in these areas is dependent on the funding of a particular project. Once the project concludes monitoring efforts tend to follow suit until the next project commences, resulting in data gaps. The Lake Victoria Fisheries Organization and Lake Victoria Basin Commission attempt to address the issue of fragmentation by including a harmonization function into its mandate, which will be discussed in Chapter Five. To alleviate the impacts of this problem, policymakers can advocate for the necessary resources required by scientists in order to answer the kinds of questions policymakers need answered (Interview, April 24, 2009, Toronto, Canada).

Fragmented policies and research investments have the potential to exacerbate the divide among the science and policy communities within and between states. Disjointed management activities and research outcomes can produce divergent views on the same issues and in other cases, may lead to disagreements on priority issues. 


\section{Political 'Readiness'}

In the majority of cases, the most serious obstacle in water management is political, not technical. Without a political setting conducive to real transition, there will be little determination for the development of transboundary water laws and policies (Bernauer, 2002; Centre for River Basin Administration, 2005; White, 1998). It was observed in the preceding chapter that the extent to which management regimes can evolve, adapt and improve is highly influenced by the political 'readiness' of the riparian states. The literature and interviews revealed that a lack of trust, knowledge imbalance and competing interests, all of which can affect the political readiness of the riparian nations and consequent degree of collaboration among countries, could be worsened by a weakened science-policy interface. It was also suggested that science could indirectly influence the extent of collaboration among states in transboundary water areas by facilitating a common understanding of the state of the water system and priority issues facing the region. This information can lead to mutual recognition of shared responsibility and governance, which is an important component for effective transboundary water management.

Lack of trust and meaningful cooperation between riparian states is a problem (Varis et al., 2008, p. 3). Building trust among riparian nations is an essential part of designing and implementing a successful transboundary water management system. While it can be difficult to initiate, achieve and sustain negotiations for water management, sharing knowledge on the state of the lake ecosystem can be the first step in designing a management system and building mutual trust. Riparian countries benefit from having a mutual understanding of the shared resource and are usually willing to exchange information (Interview, March 26, 2009, Toronto, Canada). Despite this, there are still cases around the world where information is concealed and 
not shared to protect national security (Interview, March 26, 2009, Toronto, Canada), leading to knowledge imbalance between countries. In the "political model where information is used strategically, information will be regarded as a source of power, which will in turn lead to secrecy" (Timmerman \& Langaas, 2005, p. 184). The knowledge imbalance can lead to power and capacity asymmetries and result in unequal magnitude and distribution of benefits from cooperation (Sadoff et al., 2002, p. 10).

Grey and Sadoff (2002) suggest there will always be political tensions between riparian states arising from competing agendas and that cooperation or lack thereof can influence the level of that tension. For example, a challenge for basin development is the sharing of benefits and for that matter, losses and doing it fairly. Quantifying these benefits and tradeoffs can provide the information necessary to bridge the gap between riparian countries and to inform the dialogue necessary for transboundary water management.

The complexities associated with transboundary water management can exacerbate the divergence between science and policy as illustrated by the challenges arising from fragmented policies and research efforts, communication and political differences. By the same token, a weak science-policy interface can aggravate existing tensions within transboundary areas. Given these observations, I suggest it is necessary to strengthen this interface for successful transboundary water management. In the next section, I identify some tools and mechanisms that have been adopted by various transboundary water management regimes as means of narrowing the gap. 


\subsection{Forging a Stronger Connection}

In areas of shared resources, establishing a neutral platform for cooperation is essential for international negotiations. Political, cultural and language differences can create barriers between countries, leading them to view each other as suspect. In addition, variances in resource capacity can result in varying degrees of management activities and information production. Reinforcing the role of science in these areas allows the key actors to establish their credibility and provides a means for building the mutual trust essential for developing transboundary management strategies. Scientific knowledge has the potential to reduce uncertainty between governments and create a safe environment for deliberations. Demonstrating the ecological and economic benefits of collaboration can further reduce political tensions and allow partners to overcome their differences (Grey \& Sadoff, 2002; Timmerman \& Langaas, 2005).

Integrating science into transboundary water management processes has the added benefit of revealing new intellectual relationships among the countries, which may offer new opportunities to come to compromise, deals and agreements (Lorenz et al., 2001b). Enhancing scientific understanding of an issue can also offer clarity on the roles and responsibilities of the various players in transboundary water management and in some instances, the identification of new actors for management actions. Further, establishing strong science-policy relationships increases the understanding of the issues, allowing the right questions to be asked in order for the right information to be produced (Interview, April 24, 2009, Toronto, Canada).

A number of tools and mechanisms could be adopted to strengthen the role of science in the policy process in transboundary water management regimes. Some of these have been identified in Chapter Two for narrowing the science-policy gap in environmental policy processes generally, including communication tools and stakeholder engagement. A brief 
discussion on these instruments is provided to illustrate how they could be applied in the transboundary water context. Other tools and mechanisms include investments in regional monitoring and information systems, and the creation of organizational structures.

\section{Enhanced Conmunication}

This paper previously described how communication challenges can become aggravated in transboundary water areas as a result of political, cultural and language differences. Opening and sustaining communication channels is necessary to overcome these differences and to accelerate the implementation of transboundary water programs (GWP, 2009). Effective communication is also essential for cultivating stronger relations among scientists and policymakers in different administrations for the development of harmonized policies and standards.

Creating a "communication gateway" can serve as a catalyst for cooperation and is very important in the process of conveying information (Timmerman \& Langaas, 2005; Grey \& Sadoff, 2002). Creating information exchange opportunities increases personal interaction and enhances communication between scientists and policymakers. Conferences and workshops have the ability to convene participants from various stakeholder groups and from various jurisdictions, and is an important tool for disseminating information. This is particularly valuable in transboundary areas where there are a multitude of actors. Creating a venue for information exchange and collaborative learning is instrumental in building and sharing knowledge, and the trust necessary for multilateral partnerships (GWP, 2009). For example, the GEF funded 'Twinning Workshop' convened the commissions of the North American Great Lakes and Lakes Victoria and Tanganyika to share experiences, best practices and challenges in 
September 2008. A scientist who participated at the workshop revealed that prior to this workshop, the Ministers of the Lake Victoria basin countries had not convened in a long time.

Inherent to transboundary water management is the challenge of communicating pertinent information to the multitude of individuals who have a stake in the sustainability of the resource, including constituents, industry members, community and non-government organizations, academe and government officials such as agency scientists and policymakers and political leaders. In some cases, overcoming this problem requires tailoring information to a specific stakeholder group. In other instances, the development of indicators is a simple way to convey information on the state of the ecosystem to a broader audience.

Indicators are defined as a variable or an aggregated set of variables giving information on a system, process and/or state of the ecosystem at issue. They are scientifically based and are representative of the problem (Lorenz et al., 2001a). Indicators can satisfy the information needs of policy and decision-makers and define their information needs to guide scientists in research efforts (Lorenz et al., 2001a). Indicators have the ability to condense the profusion of data and information into manageable and understandable formats to simply convey information to various stakeholders. For example, the State of the Lakes Ecosystem Conferences (SOLEC) report provides information on the state of the Great Lakes ecosystem and the major factors affecting it through a suite of approximately 80 indicators. It also provides a forum for information exchange amongst Great Lakes decision-makers and a means to communicate information to people in all levels of government, corporate, and not-for-profit sectors that make decisions that affect the Great Lakes (Environment Canada \& USEPA, 2010).

Indicators can also be used to evaluate management activities. For example, the GEF established a set of indicators to monitor the progress of GEF funded projects. Process indicators 
are identified as the most important indicator of success in GEF international waters intervention (Duda, 2002). These indicators characterize the completion of institutional processes that will result in joint action on needed policy, legal and institutional reforms and investments. Examples of process indicators include the adoption of a science advisory panel or the adoption of a monitoring and evaluation plan during project preparation (Duda, 2002). There are, however, concerns that there are tradeoffs between scientific robustness and simplification for management and policy needs with the use of indicators (Lorenz et al., 2001a; Duda, 2002).

\section{Stakeholder Engagement}

As transboundary water management regimes are moving toward an integrated water resources management approach, stakeholder participation is becoming a critical component for the development of effective policies. In some areas, public engagement is routine and is institutionalized in management processes whereas in others, stakeholder involvement is incipient. The majority of experts interviewed identified the inclusion of stakeholder consultations as an important step in the policy process. Some indicated that public engagement is particularly significant in transboundary water areas as their participation can facilitate policy implementation across jurisdictions. In some instances, non-government organizations can also enhance the credibility of lake managers.

Given that stakeholder participation is identified as a means of narrowing the sciencepolicy gap and as a criterion for effective transboundary water management, one can expect that adopting tools that enhance stakeholder involvement would forge stronger relations between the science and policy realms in transboundary water management. This is evident in the Great Lakes management regime where non-government organizations are at times procured to conduct scientific studies to inform policy development (Interview, April 24, 2009, Toronto, 
Canada). NGOs can be requested to undertake research to confirm or refute scientific knowledge produced by agency scientists, which could enhance the credibility of the information.

Public participation provisions in transboundary agreements can ensure stakeholders are consulted in the development of policies. The 2003 Convention on the Sustainable Development of Lake Tanganyika includes a proviso for public participation in the decision-making process. The basin countries of Burundi, Congo, Tanzania and Zambia agreed to adopt and implement legal, administrative and other appropriate measures to ensure the public has the right to participate in the decision-making process, including activities related to environmental assessments. The public is also given the opportunity to make oral or written representations before a final decision is taken on a particular project or activity. National Inter-ministerial Committees have been established to guide and supervise implementation activities and to ensure civil society and private sector all participate in lake management decision-making processes (Bihamiriza, 2007).

Governments can also create formal channels for stakeholders to provide advice directly to policy and decision-makers by establishing advisory committees and boards with participation by members of the public. For example, in addition to government agency scientists, researchers from academe and industry from Canada and the United States are represented on the Science Advisory Board (SAB) of the International Joint Commission (IJC) (Botts \& Muldoon, 2005, p. 19). The $\mathrm{SAB}$ has permanency in the commission and interfaces with the Great Lakes Water Quality Board (WQB) to inform policy recommendations. There are also transient advisory bodies that may be established to advise governments on critical issues. The IJC's creation of the Lake Erie Task Force in 1993 is such an example. The Task Force advised the Commission on 
the impact of various stressors affecting the benthic and fish communities in Lake Erie. It concluded its mandate in 2006 (IJC, 2010). These are some examples of ways in which nongovernment participation could be enhanced in the policy development process in transboundary water management regimes.

\section{Investments in Regional Monitoring and Information Systems}

A consistent concern expressed by the interviewees and identified in the literature is the lack of monitoring data. This risks producing ineffective and potentially erroneous policies. Investments in monitoring are therefore necessary for the development of sound management practices for environmental protection. In transboundary water areas, this requires greater effort and collaboration by all riparian states as fragmented monitoring investments can lead to disparate data sets and inconsistent information and in some cases, can exacerbate political tensions. Establishing regional monitoring networks could mitigate these challenges (Hecky et al., 2006, p. 207) and provide data on ecosystem changes that may affect water quality, quantity and fisheries.

Regional networks have been established in a number of transboundary water areas as a way of building scientific capacity and a means to forge stronger relations between riparian countries. For example, there have been significant efforts to enhance the regional monitoring network in the Danube Delta. A component of the Danube Regional Project funded by the United Nations Development Programme reinforces monitoring to provide information for the control of transboundary pollution and to develop and assess methodologies for monitoring nutrient reduction in wetlands.

Regional monitoring networks can also provide a way of ensuring development activities do not adversely affect the water system. In 1985 the Mekong River Commission established a 
Water Quality Monitoring Network in the Lower Mekong Basin. The monitoring data collected from approximately 100 monitoring stations are used to inform the commission and Mekong basin countries of adverse effects resulting from development activities that could jeopardize water uses (Mekong River Commission, 2005).

Investments in regional monitoring networks can provide the information necessary to guide policymakers in the policy development process. Long-term trends provide a more accurate picture of ecosystem changes. This thesis previously highlighted that poorer riparian countries are dependent on international partners for investments in monitoring and are more susceptible to data gaps as a consequence of project cycles. The sustainability of these regional networks therefore requires national governments to commit human and financial resources to monitoring activities over the long-term.

Also critical for transboundary collaboration is information accessibility. Regional information and data management systems can increase information sharing among riparian countries. These are electronic systems that arrange, store and exchange data and information and include Geographic Information Systems (GIS) (GWP, 2009). Having access to research and monitoring data can assist countries in the development of complementary and harmonized policies. Regional information systems can also enhance transparency and build trust among the countries.

The utility of this tool is recognized by member states participating in the Nile Basin Initiative through the development of the Nile Information System (NIS) (Interview, Nile Basin Initiative, June 11, 2009, Entebbe, Uganda). Upon completion, the NIS will become the clearinghouse for research and monitoring data for Nile River management. Another example is the Great Lakes Monitoring Inventory and Gap Analysis (Great Lakes Commission, 2010). The 
Great Lakes Commission engaged in this large-scale, binational initiative to collect monitoring program information from federal, state/provincial, local and non-governmental organizations across the Great Lakes region. The inventory includes more than 600 monitoring programs and is the basin's first comprehensive monitoring inventory. Using the monitoring inventory, the Commission also developed a report that assessed gaps and overlaps in monitoring programs. (Great Lakes Commission, 2010).

\section{Organizational Structures}

Organizational changes within government departments can be an effective way to strengthen the connection between science and policy. As opposed to the mechanisms and tools previously described, departmental changes require much stronger top-level commitment and understanding and recognition of the critical role that science plays in environmental policy development. These organizational changes also require decision-makers to understand and appreciate the importance of cultivating relationships between the two communities as a requisite to the overall sustainability and efficacy of the management regime.

There are examples of organizational modifications that have resulted in enhanced understanding and improved communication and collaborative undertakings between scientists and policymakers. The Canadian Government's desire to strengthen research and the role of the Canadian National Water Research Institute is one such example. The commitment to science was made in the government's Speech from the Throne in January 2001, which gave the department the political support it needed to expand the institute with the creation of the Science and Technology Branch.

The creation of commissions in transboundary water areas is another example of establishing an organizational entity to forge stronger relationships between scientists and 
policymakers. The Lake Victoria Fisheries Organization is a forum whereby scientists and policymakers from all three riparian countries convene to discuss fisheries issues and make policy recommendations to their respective Ministers. This will be discussed more extensively in the next chapter. In both these examples, the impetus for organizational change was in response to an environmental crisis - a window of opportunity.

Some causes for the divergence between science and policy are similar to environmental policy processes generally, yet can be exacerbated in transboundary areas. Issues of politicization of science and communication are more complex due to the magnitude and number of issues and actors. Other reasons are more characteristic of joint management regimes including uncoordinated research efforts, which lead to inconsistent data collection and reporting methods, and consequently fragmented management policies between riparian countries. Similarly, one can also draw parallels with the tools and mechanisms that enhance communication and public participation in transboundary areas. To address the issue of policy and research fragmentation requires national and sub-national governments to invest in regional monitoring and information systems. Further, in circumstances where political and senior level commitment is secured, the creation of organizational structures to facilitate science-policy dialogue is a very effective means to strengthen the interface.

In the next section, the tools and mechanisms used to integrate science into the Laurentian Great Lakes management regime will be briefly discussed. This will illustrate the practical application of the theories and instruments previously identified in an area that is well established and internationally renowned. 


\subsection{Approach to Managing the North American Great Lakes}

With over 260 international water bodies in the world, there are a comparable number of management regimes. Some are deemed to be a success and others to a lesser extent, and in some cases, there have been unsuccessful attempts to develop management strategies as was observed with Lake Chad (Hodge, 2006). To illustrate the science and policy dynamics in an existing management regime, this paper briefly examines the approach taken in the Laurentian Great Lakes. The Great Lakes management regime, when compared with other areas, has an abundance of resources and strong political commitment. It is one that has evolved over a century and is suggested by some scientists and policy experts as internationally renowned and a success (Interview, April 18, 2009, Windsor, Canada). This observation notwithstanding, the extent of its success is debatable. It has been observed that transboundary and domestic policy efforts have not resulted in improvements in some of the most polluted sites in the Great Lakes (Johns, 2010, p. 101). This discussion offers some comparative perspective for the case study on East Africa's Lake Victoria.

The North American Great Lakes make up twenty per cent of the world's freshwater resources and are an important national, continental and international resource (John, $2009 \mathrm{p}$. 96). Throughout history, the lakes have been used for a number of different purposes including transportation, hydroelectricity, fisheries, water supply and waste disposal (Sproule-Jones, 2002; Johns, 2009). These activities persist, requiring lake managers and scientists to continue working collaboratively to ensure the resource is sustainable for current and future generations.

The Great Lakes management regime is well established, recently celebrating the one hundredth anniversary of the Boundary Waters Treaty that initially created the governance structure for lake activities. The Canadian, United States and sub-national governments have 
dedicated significant resources to lake management activities. This includes investments in scientific capacity, which has bestowed upon the management regime a strong level of credibility with government agencies and the public.

The regime consists of a multitude of players, and encompasses a number of interconnected management schemes that have arisen from national, sub-national and local levels. It is not my intent to explore all these management systems but rather to illustrate some examples of how science can be integrated in the policy process and the effects this has had on policy and management outcomes. This is primarily done from the perspective of the International Joint Commission and the Great Lakes Fishery Commission, as well as from the perspective of a sub-national government, the Ontario Ministry of the Environment.

\section{Science and Policy in the North American Great Lakes}

Although significant resources have been dedicated to build scientific capacity in the region, and considerable effort to cultivate strong relations among the science and policy communities, the Great Lakes management regime is not immune to the challenges that are characteristic of transboundary areas. Scientists and policymakers involved with Great Lakes management indicated the system continues to suffer from issues arising from ineffective communication and ambiguities in scientific results. Further, it was noted that existing governing structures, as a result of their maturity, do not allow the system to adapt easily. "Some organizations are calcified and are not amenable to change" (Interview, April 8, 2009, Windsor, Canada). Additionally, it was noted that some Great Lakes programs have maximized their potential and are not producing any further benefits to the lakes. Notwithstanding these challenges, the Great Lakes management regime is internationally recognized as a progressive and credible model that 
has produced measurable improvements in lake quality including the reversal of eutrophication in Lake Erie.

Since the establishment of a Great Lakes bilateral governance structure, science has had an important function in the development of agreements and policies, and for providing the necessary information to resolve conflicts and strengthen partnerships. The Great Lakes scientists and policy experts interviewed for this study highlighted that a strong science-policy connection is a key element in the success of management efforts. Notwithstanding this desire, there have been instances where political interference influenced the extent of scientific integration into the policy process. This was demonstrated in the development of the Hamilton Harbour Remedial Action Plan where a science expert, who was also an academic, was asked to withdraw from the investigations by a local politician (Sproule-Jones, 2002, p. 16).

Great Lakes governance is rooted in a number of binational agreements including the 1909 Boundary Waters Treaty and the 1972 Great Lakes Water Quality Agreement (GLWQA), which was subsequently renewed in 1978 and amended in 1987. The GLWQA is currently under another review and negotiations of amendments to the agreement formally commenced on January 27,2010 . A number of these binational agreements are based heavily on scientific objectives and outcomes (Sproule-Jones, 2002, p. 42).

As an example, the 1972 GLWQA was established in response to the 1964 lower lake reference that revealed Lake Erie was undergoing eutrophication (Botts \& Muldoon, 2005; Johns, 2010). Scientists initiated a binational fact-finding mission that "largely led [to the] development of the 1972 agreement because of scientific consensus that reduction of phosphorus loadings could slow eutrohication" (Botts \& Muldoon, 2005, p. 52). This revelation spurred nations to act fervently to develop and implement programs and measures for the reduction and 
control of inputs of phosphorus and other nutrients. In addition, a number of provisions also make reference to science including Article V on Standards, Other Regulatory Requirements and Research. Formally instituting science into transboundary water agreements ensures it is considered in the development of policies to meet the objectives of the agreement. However, an Environment Canada representative cautioned that without a regulation to enforce the commitments under the agreement, implementation could be a challenge (Interview, March 25, 2009, Toronto, Canada).

Organizational structures are also important in strengthening the science-policy connection. In the Great Lakes management regime, "there is always a desire to strengthen the way in which science feeds Great Lakes programs. The usual avenues are through a committee or some other structure as formalized in the bureaucracy" (Interview, March 25, 2009, Toronto, Canada). The example that illustrates this most effectively is the creation of the International Joint Commission (IJC) under the Boundary Waters Treaty.

The IJC was established to prevent and resolve disputes between Canada and the United States and to pursue the common good of both countries as an independent and objective advisor to the two governments (IJC Website, 2010). Within the IJC, there exist a number of advisory boards including the Science Advisory Board (SAB) and the Water Quality Board (WQB). Recently, the SAB and WQB integrated their activities, allowing policy and resource management implications to be front and centre of research needs (Interview, April 8, 2009, Windsor, Canada).

It has been suggested the IJC developed its strong reputation through credible science, its objectivity and non-partisan stance (Sproule-Jones, 2002; Botts \& Muldoon, 2005). However during the 1990s, the IJC lost some of its effectiveness in securing support for its 
recommendations (Sproule-Jones, 2002, p. 48). An IJC scientist interviewed for this study confirmed this observation. It was revealed that in the 1990 s, the IJC did not stay rooted in science and called for the sunsetting of chlorine without the necessary scientific evidence to justify the position. The SAB and WQB were working on organochlorine compounds at the time, but did not recommend to the Commission the sunsetting of all chlorine compounds. The IJC ignored the advice of their advisory boards and instead sided with ENGOs on this issue, resulting in eventual lack of confidence in the IJC by the national and subnational government agencies. It was noted by this individual that a key lesson learned from this experience is to ensure the work undertaken by the commission is scientifically sound from the outset in order to achieve success (Interview, April 8, 2009, Windsor, Canada). This is understood to mean that the commission should remain rooted in science and refrain from being influenced by political or public interests in order to maintain its objective stance.

Although the IJC strives to base its work on the most sound scientific information, it has also been suggested that while the IJC "once generated significant research and policy responses...[it has] not provided the results required to address persistent water pollution problems associated with multiple uses in the Great Lakes (Johns, 2010, p. 118). Further, the institutional weakness of the IJC is its dependency on the cooperation of national and subnational governments for the implementation of its recommendations and lack of enforcement authority (Sproule-Jones, 2002, p. 47).

The Great Lakes Fishery Commission (GLFC) is another example of a transboundary organization that has successfully strengthened the linkage between science and policy. The GLFC was established in the 1950 s to support a binational effort to eradicate the invasive sea lamprey and to coordinate fishery management programs and policies. As part of these efforts, 
the GLFC has developed a Science Transfer Program to promote communication of information about Great Lakes ecosystems and their fish communities to fishery researchers and managers and to the public. Science transfer projects can include activities that disseminate information such as science-transfer workshops and scientific review papers. Further, the GLFC is the facilitator of the Joint Strategic Plan for the Management of the Great Lakes Fisheries, a nonfederal, multilateral, nonbinding agreement. The committee structure of the Joint Strategic Plan promotes the translation of science into management. It does this by including at least one technical committee to provide scientific advice for every lake committee (Gaden \& Krueger, 2010, p. 102).

The role of non-government participants has been highlighted throughout this paper as a critical component to the management of transboundary waters. Strong public participation promotes government transparency and accountability (Interviews, 2009; Sproule-Jones, 2002). The Great Lakes management regime has taken significant strides to build a strong public participation component in lake activities. The IJC's biennial meetings are an example of a mechanism that creates a forum for the public to voice their concerns. The public views the Commission as their vehicle to the heads of state in Canada and the United States (Interview, April 8, 2009, Windsor, Canada). Sub-national governments have also made public consultation a routine part of policy development processes. The Ontario Government's Environmental Registry is a mechanism established under the Environmental Bill of Rights. It is designed to inform the public on new and amended regulations and legislation, including those designed for Great Lakes management. It promotes transparency and accountability of government actions and provides a means for stakeholders to submit comments on the proposed legislation and regulations. 
In addition, some non-government participants including research and academic water networks in Canada have Great Lakes research agendas, such as the International Association for Great Lakes Research and the Canadian Water Network (Johns, 2010, p. 103). These research organizations can assist with building scientific knowledge. NGOs also can help translate technical information and policy objectives to the public, which not only enhances the understanding of science but can also produce the support to drive public policy. A strong public constituency has the power of persuasion to guide public policy development. As a Great Lakes policy expert highlighted, "NGOs are our biggest allies. We can make the science and policy case to act, but unless there is a strong public appetite for it, it is hard to get the issue to the top of the agenda" (Interview, April 24, 2009, Toronto, Canada).

Finally, recognizing that communication challenges are common to all transboundary water management areas, and environmental policy processes for that matter, I take this opportunity to discuss some of the tools and mechanisms employed by Great Lakes managers and scientists to enhance communication. Great Lakes scientists and policymakers highlighted the translation of science and the incessancy of working within silos as issues experienced in the Great Lakes regime.

National and sub-national departments are directing efforts toward 'in-reaching' activities. As opposed to outreaching, these efforts attempt to engage and educate staff within the government institution on scientific issues, including those of emerging concern. As IWRM becomes increasingly important in the management of transboundary waters, integrating the various organizations that govern land use and agriculture for example is critical to the overall management of the water body. A tool developed for this purpose is issue papers that focus on a range of science topics and are disseminated to a variety of audiences within an administration. 
A policy expert with the Ontario Ministry of the Environment also highlighted the creation of unofficial 'inner teams' of mostly Great Lakes scientists and some policymakers who convene regularly to discuss the state of the science and policy direction. These regular interactions forge stronger connections between the two communities and have the potential to create a resilient 'neuro-network' of connections (Interview, April 24, 2009, Toronto, Canada).

Creating information exchange opportunities is also important in strengthening the science-policy interface. Conferences for example, can engage participants that are not traditionally involved with the management of the Great Lakes such as those in agriculture, land use and transportation. The aforementioned State of the Lakes Ecosystem Conference is a forum that convenes lake managers and scientists to discuss priority issues and solutions facing the Great Lakes. These conferences were designed to meet the requirements of the GLWQA for a report on the Great Lakes every two years (Botts \& Muldoon, 2005, p. 174). Through the use of indicators, the conference develops a binational picture of the state of science on very specific issues (Interview, March 25, 2009, Toronto, Canada). It has been suggested, however, that SOLEC should go beyond reporting on the state of the lake and produce environmental management recommendations in order to affect change (Botts \& Muldoon, 2005, p. 174).

Another example of an innovative project that has successfully forged a stronger sciencepolicy connection is the International Association for Great Lakes Research (IAGLR). Through IAGLR's Science-Policy Project, the science-policy linkage was strengthened in three ways (Botts \& Muldoon, p. 195): 
(1) A directory of IAGLR experts was established and made available through the World Wide Web. These experts agreed to be available to answer questions from policymakers;

(2) Back issues of the IAGLR Journal of Great Lakes Research was made available electronically; and

(3) Three policy papers were released on major ongoing and emerging threats to the Great Lakes. These papers summarized the state of scientific understanding on the topic and laid out specific policy proposal.

As one can observe, the Great Lakes management regime has committed considerable resources to enhance the role of science in management activities. Even during the incipient stages of Great Lakes management, lake managers and scientists recognized the importance of science in the development of Great Lakes policies. This is evident in the agreements established to govern lake activity and research investments that led to the revelation of eutrophication in Lake Erie for example. The regime has also committed to strong stakeholder involvement in the design and implementation of policies and programs. These efforts have strengthened the science-policy interface in the Great Lakes management regime and enhanced its credibility with both domestic and international partners. Conversely, ignoring the science not only risks producing ineffective and erroneous policies but can also result in a loss of credibility and trust with stakeholders as illustrated with the International Joint Commission. Even though the Great Lakes management regime is well established, it continues to suffer from communication problems between science and policy communities within and across administrations.

The experiences of the Great Lakes regime can offer some lessons about the integration of science in transboundary management. Great Lakes governance has evolved over a century and has demonstrated that science has the potential to enhance or reduce the credibility of a 
system and the ability to influence policy outcomes. These lessons can inform the development of emerging transboundary management regimes, including that of Lake Victoria. 


\section{Chapter 5: Lake Victoria: A Case Study on an Emerging Transboundary Water MANAGEMENT Regime}

\section{Introduction}

Lake Victoria is the second largest freshwater lake by surface area, second only to North America's Lake Superior. It is nestled between Tanzania, Uganda and Kenya, each having 49, 45 and 6 per cent jurisdiction over the lake surface, respectively (Ntiba, Kudoja \& Mukasa, 2001). These countries remain among the poorest countries in the world. Burundi and Rwanda lie within the lake drainage basin and have recently been recognized as significant parties for integrated lake management. The Kagera River, which drains Burundi, Rwanda and part of Uganda, is the largest river flowing into Lake Victoria.

Lake Victoria supports approximately 30 million people in the basin. Population density in the area is above the national average in all the countries. Growth rates in the riparian communities are six per cent per annum and are among the highest in the world (Klohn \& Andjelic, 2008; Kayombo \& Jorgensen, 2006).

Lake Victoria covers nearly $69,000 \mathrm{~km}^{2}$, with rather shallow mean and maximum depths of $40 \mathrm{~m}$ and $79 \mathrm{~m}$, respectively and a volume of $2,760 \mathrm{~km}^{3}$ (Odada \& Olago, 2006; Kayombo \& Jorgensen, 2006). Its shallow depth allows the lake to be very productive, which is characteristically similar to Lake Erie in North America. Much of the lake shoreline is indented, with many large and shallow swampy bays that occupy a high proportion of the total surface area (Kairu, 2001). Lake Victoria stretches $412 \mathrm{~km}$ from north to south and $355 \mathrm{~km}$ west to east. Approximately 86 per cent of total water input falls as rain with very high evaporative losses of 80 per cent (Kayombo \& Jorgensen, 2006). The lake's water residence and flushing times of 23 and 123 years - both of which contribute to its long retention time - allow pollutants entering the 
lake to remain in it for a long period. The size of Lake Victoria can give rise to the misconception that the lake is 'too large to pollute'. Previous experiences in the North American Great Lakes have demonstrated that size alone does not protect the resource from environmental degradation resulting from rapid economic development (United Nations University, 2008).

\subsection{History and Significance}

To some, Lake Victoria is a "God given treasure" (Interview, May 26, 2009, Jinja, Uganda) to the riparian communities in the region. It offers a multitude of goods and services that serve beyond national boundaries. Lake-derived exports, particularly fish species such as the Nile perch and tilapia, have increased dramatically over the past several decades. The lake feeds into the Nile River, supporting countries along the Nile through hydropower generation and providing the necessary resource for extensive irrigated agricultural schemes. The pressures on Lake Victoria are already gargantuan. Existing pressures on Lake Victoria are expected to intensify as populations and economies grow, and lake quality and quantity deteriorate.

In recognition of the potential risks that may plague the region in the future, recent investments have been made to sustainably manage Lake Victoria. The extent the lake can be sustained and support current and future activities will depend on the degree that riparian countries can effectively collaborate and manage this shared resource. This thesis argues that strengthening the science-policy interface is a building block for the development of an effective Lake Victoria management regime. This is done so by examining the science-policy connection and the role of science in this area. It is also recognized there are a myriad of factors that influence the outcomes of policies in this region and that science is just one factor that can influence management outcomes. 
Prior to the discussion on the Lake Victoria science-policy interface, some context about the ecological and socio-economic significance of the lake is provided. This paper then highlights some of the more notable ecological pressures facing Lake Victoria and concludes the discussion by providing an overview of the historical and existing governance structures established to manage this shared resource.

\section{Ecological and Socio-Economic Significance of Lake Victoria}

Lake Victoria serves a multitude of purposes and communities in, around, and beyond the basin. The lake's riparian communities depend on the lake and its resources for survival. Subsistence fishing and farming are characteristic of the types of livelihood available to riparian communities. The lake is also important for residents in urban environments as well as industries as it is their primary source of water supply. In some instances Lake Victoria is used as a repository for human, agricultural and industrial waste. Lake Victoria is also the lifeline for Nile basin countries and provides hydropower through its only outlet at Owen Falls in Uganda and to other power plants downstream (Kayombo \& Jorgensen, 2006). Further, Lake Victoria offers goods and services that continue to serve global communities as exemplified with increasing exports of the Nile perch and tilapia. In addition to anthropogenic benefits, the lake also provides a protective habitat for some of the endangered haplochromine species, hippopotamus, crocodile and snakes (Odada \& Olago, 2006).

The industry of most socio-economic significance to the region is fisheries. Lake Victoria supports the world's most productive freshwater fishery (UNEP, 2010), which is predominately based on the Nile perch (Lates niloticus) and tilapia (Oreochromis niloticus). A Lake Victoria scientist characterized the lake as an insurance policy for the people as there is always something that can be caught (Interview, May 26, 2009, Jinja, Uganda). The fisheries 
industry is currently the most important non-traditional export commodity, yielding more than 300,000 tons annually worth US\$600 million per year (Kayombo \& Jorgensen, 2006). It employs approximately 136,000 artisanal fishermen and over 700,000 people are involved in activities along the production to consumption chain (NaFIRRI, 2009, p. 8). The fisheries sector contributes 5-12 per cent of the gross domestic product in the region (NaFIRRI, 2009, p. 8). Although exports generate significant earnings on a national scale, the average income per capita is only a fraction of this, ranging from US\$90 to US\$270 per annum (Klohn \& Andjelic, 2008).

The Lake Victoria ecosystem has undergone substantial and, to some observers, disturbing changes that have accelerated over the last three decades (Kayombo \& Jorgensen, 2006). Multiple activities in the lake basin have led to an alarming degradation of soil, water, wetlands, and fragile ecosystem conditions; loss of fish populations; and rising resource management costs (Barghouti, 2006, p. 1). Rapid population growth, over fishing, introduction of predatory fish species, pollution from industrial and agricultural sources, eutrophication, noxious waterweeds such as the water hyacinth, and degraded wetlands are all threatening the sustainability of the lake. Climate change and increasing competition for lake resources by countries along the Nile River will inevitably intensify these pressures (Klohn \& Andjelic, 2008; Kayombo \& Jorgensen, 2006; Ntiba et al., 2001). Lake Victoria is facing a host of challenges that if left unmanaged, will permanently affect the lake ecosystem functions.

\subsection{Challenges Facing Lake Victoria}

Many of the threats facing Lake Victoria are the result of rapid population growth in the basin. Figure 3 illustrates the principal events in the recent environmental history of Lake Victoria, in relation to human-population growth and agricultural production in its drainage basin. It is evident that there is a strong correlation between population growth and agricultural activity, as 
well as the surge in other lake activities agricultural production ( $\%$ of 1960 value)

including the introduction of the Nile

perch and the eventual collapse of

indigenous fish stocks. Further,

multiple activities in the lake basin

have increasingly come into conflict

and have caused the lake ecosystem to

undergo some substantial changes

rendering the lake environment

unstable (Klohn \& Andjelic, 2008). It

is anticipated that population pressures

will continue to burden the resource as

current estimates project a doubling of

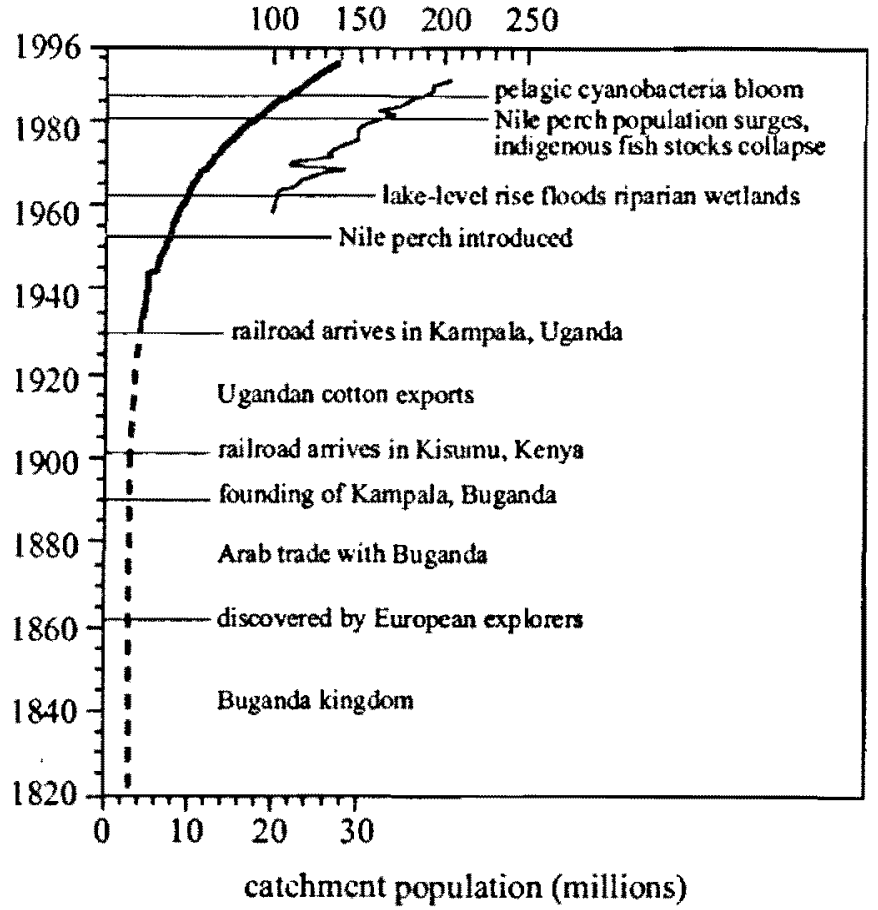

Figure 3: Principal events in the recent environmental history of Lake Victoria, in relation to human-population growth and agricultural production in its drainage basin Source: Verschuren et al. (2001)

regional human population to 53 million by the year 2020 (Verschuren et al., 2001).

According to the Food and Agriculture Organization (FAO), more than 75 per cent of the world's fish stocks are already fully exploited, overexploited, depleted or recovering from depletion (FAO, 2006). In East Africa, the lucrative nature of the fisheries industry began what many believe to be the eventual collapse of indigenous species and consequent introduction of the predatory Nile perch in Lake Victoria to replace the depleted native fish stock. The overexploitation of the fisheries sector can be attributed to an increase in fishing activity and the application and use of modern and illegal fishing technology. Further, new fishing regimes in the form of fish processing plants are also a contributing factor in the rapid decline of lake fish 
stocks. The issue of sustainable fish stocks continues to be debated in the management of Lake Victoria today.

Threats to the Lake Victoria fishing industry were observed as early as 1928 . The eventual decline in popular native species like the native tilapia in the mid-nineteenth century led to intense debates over the introduction of an exotic species to improve the state of fisheries. The Nile perch, a piscivorous or fish-eating fish native to the Nile River and Lakes Albert in Uganda and Turkana in Kenya was the primary candidate considered. Research scientists at the East African Fisheries Research Organization (EAFRO) objected to the proposed introduction and argued the introduction would have "unpredictable and potentially untoward effects on the established tilapia fishery" (Pringle, 2005). Despite their conservative arguments, in 1954 the Nile perch was 'mysteriously' introduced into Lake Victoria to address the decline of native species, and to sustain the fisheries in lake Victoria.

Pringle (2005) observed that for about 20 years after the introduction of the Nile perch, the success of the introduction was questionable. The Nile perch established itself in low densities and was infrequently caught by fishermen. In the 1970s, a sudden spike in Nile perch population was observed. Total catch rose from 100,000 tons per year in early 1970 s to about 500,000 tons in 1989 (Klohn \& Andjelic, 2008). "A new fishery is like a gold rush - the first ones in reap the biggest rewards while late arrivals may find it much harder to make money" (Dr. Brian Marshall, Resource Monitoring Specialist, LVFO, May 26, 2009, Jinja, Uganda). This significant increase shifted the fishery industry from local to global markets and resulted in a tremendous benefit for national and local economies of Kenya, Tanzania and Uganda (Pringle, 2005). The introduction improved export earnings for the region and increased employment and earnings for local fishermen. 
In response to the profitable fisheries industry, the number of fishers on the lake increased substantially. It is estimated that there are presently 200,000 fishermen on the lake (Interview, May 26, 2009, Jinja, Uganda). Fisheries in Lake Victoria have historically been unrestricted; anyone can make or buy a vessel and start fishing without much attention paid to the number of fishers on the lake and the means used to catch the resource (Ntiba et al., 2001). This has led to a consequent influx of fishers employing illegal, unregulated and destructive fishing practices. In some cases, fishers have resorted to the practice of fish poison. It was not until human deaths due to fish poison were reported in 1999, and the resulting ban of fish imports by the European Union, that authorities took action (Ntiba et al., 2001). The recent creation of Beach Management Units (BMUs) in the basin is an attempt by governments to control the number of fishers on the lake by requiring them to register with a BMU. A Fisheries Officer at the Gaba landing site near the capital city Kampala shared that in principle, "any person cannot access the lake unless he has been registered with a BMU. And when it comes to enforcement of regulations and rules, the BMUs are now the lead agencies in monitoring, control and surveillance" (Interview, June 9, 2009, Kampala, Uganda). BMUs secure some of their funding through the fisheries industry as well as through other means such as member fees, landing site user fees and fines levied for by-law infringements to carry out these activities. Notwithstanding this intent, another respondent revealed a disconnect between what is intended and what is in practice. "We were in this remote part of the lake and we asked [the fishers] what BMU they were part of and they had no idea what we were talking about" (Interview, April 17, 2009, Toronto, Canada).

The modernization of fishing technology and methods, and the creation of new fishing regimes in Lake Victoria are also contributing factors to the decline of the lake's fish stocks 
(Kateregga \& Sterner, 2009; Balirwa, 2007). The Nile perch supports approximately 30 fish processing factories in the riparian states (Kayombo \& Jorgensen, 2006). Fish processing plants were established to meet global demands for the Nile perch and tilapia and have contributed to the rapid decline of fish stocks in the region. There is concern that the activities of the fish processing plants are simulating fishing patterns observed in the lake in previous decades that led to the collapse of the native fisheries.

There are also concerns that current sizeable fish catches may not be sustainable. Fisheries managers have observed a decline in Nile perch stocks from 790,000 tons in 1999 to 530,000 tons in 2001 (Kayombo \& Jorgensen, 2006). If this trend continues, the fisheries sector will either collapse or undergo substantial changes. The average size of fish being caught is also diminishing in some areas, further exacerbating the fish stock crisis as fish are unable to mature and breed (Ntiba et al., 2001).

Concerns over depleting fish stocks in Lake Victoria are resulting in growing interest in aquaculture. While this practice, along with the possibility of introducing other exotic species into the lake can increase production, Balirwa (2007, p. 120) emphasizes that "such interventions require firm commitment to sound ecological principles and strict enforcement of recommended conservation and co-management measures in capture fisheries." Failing to do so may result in unintended consequences as experienced with previous fisheries management efforts.

While the introduction of the Nile perch has resulted in tremendous economic benefits to the riparian states, the ecological consequences have been devastating and in some respects, irreversible. Its increased numbers had two unintended consequences: fewer endemic fish and less biodiversity. As recently as the 1960s, the lake supported an endemic cichlid fish species flock of over 500 species. These cichlids or haplochromines were classified as "trash" fish and 
were thought to be of little socio-economic value to the riparian states. As the Nile perch increased in number, they preyed voraciously on the haplochromines resulting in the disappearance of hundreds of species (Pringle, 2005; Odada \& Olago, 2006); this ranks as the largest single recorded vertebrate extinction attributable to specific human actions on earth (Odada \& Olago, 2006). As haplochromine populations became depleted, juveniles of other fishes and macro-invertebrates became prey of the Nile perch (Balirwa, 2007). What lake managers did not expect was the radically altered trophic structures in the lake as a result of declining native species (Ntiba et al., 2001). "The introduction of the Nile perch in Lake Victoria has been treated as a textbook example - literally - of the disastrous consequences of environmental management" (Pringle, 2005, p. 512).

Pollution is also a major problem in the Lake Victoria basin. Pollution pressures are increasing and the impact by municipal and industrial discharges is visible in some of the rivers feeding the lake and along the shoreline (Klohn \& Andjelic, 2008). There is generally a low level of awareness of environmental conservation and protection among industrialists (Ntiba et al., 2001). This can result in direct discharges of effluents into the lake without understanding or recognizing the potential impacts of pollutants on the ecosystem.

Further, the estimated doubling of the region's population by 2020 is a concern for lake managers attempting to control pollution sources. As point sources are identified and regulated, including breweries, tanning and fish processing plants, non-point sources are becoming increasingly significant to the overall pollution load to the lake. Efforts to control non-point sources are more challenging and require broader public recognition and participation.

Eutrophication is also an issue in African lakes but is more pronounced in Lake Victoria (Odada \& Olago, 2006). The lake's long retention time allows pollutants entering the lake to 
remain for a long period of time. Land use patterns rather than food-web alterations or climate change is proposed as the dominant cause of the ongoing eutrophication experienced in Lake Victoria (Verschuren et al., 2001). Land use activities can contribute to eutrophication through nutrient runoff, leakage to surface water and increased nutrient release to the atmosphere from animal and biomass burning and deposition to surface water (Kayombo \& Jorgensen, 2006). Some experts consider atmospheric deposition as the single most important contributor to nutrient loading. It has been reported that atmospheric deposition could contribute 49 and 63 per cent, respectively, of the nitrogen and phosphorus load in Lake Victoria (Kayombo \& Jorgensen, 2006; Odada \& Olago, 2006). Some authorities however question these data.

Increased nutrient loading of phosphorus and nitrogen can result in amplified algal growth. It has been reported that algal growth has increased five-fold since the 1960s in Lake Victoria (Klohn \& Andjelic, 2008) - a symptom of eutrophication, which could result in 'dead zones'. There have been reported areas of deoxygenation to within $30 \mathrm{~m}$ of the lake surface as a result of eutrophication (Njiru, Kazungu, Ngugi, Gichuki \& Muhoozi, 2008; Kayombo \& Jorgensen, 2006). Given Lake Victoria's mean depth of $40 \mathrm{~m}$, this implies a significant volume of the lake could be unsuitable for fish habitat, at least for part of the year. With current estimates projecting a doubling of the region's population by the year 2020, basin-wide efforts by all five countries is critical to mitigate the effects of eutrophication and to prevent further degradation of the Lake Victoria ecosystem.

In 1988, Lake Victoria also suffered from the impacts of the water hyacinth invasion. An explanation for the introduction and eventual proliferation of the water hyacinth is still unknown although it has been suggested the source of the invasion is the Kagera River, the main tributary to the lake (Kateregga \& Sterner, 2009; Klohn \& Andjelic, 2008). The water hyacinth 
infestation reached its peak in 1998 when 12,000 hectares of water were covered by the weed. Of this, 6,000 hectares were in Kenyan waters, 2,000 hectares in Tanzania, and 4,000 hectares in Uganda (Kayombo \& Jorgensen, 2006).

The effects of the water hyacinth mats were felt throughout the Lake Victoria basin. The waterweed choked many bays and inlets and interfered with electricity generation at Uganda's Owen Falls power station. It also invaded fishing grounds and blocked water ways, interfered with lake transport of irrigation and drainage water in canals and ditches, hindered navigation, increased sedimentation by trapping silt particles, decreased human food production in aquatic habitat, increased the costs of water supply, decreased possibilities of washing and bathing and adversely affecting recreation (Ntiba et al., 2001; Kayombo \& Jorgensen, 2006; Kateregga \& Sterner, 2009). Water hyacinth would reduce sunlight penetration and lower oxygen content in the water, and consequently reduced floral and faunal diversity (Odada \& Olago, 2006). It also provided a preferred habitat for bilharzias and mosquito vectors for malaria (Klohn \& Andjelic, 2008, p. 3). Despite all of these negative outcomes, one of the positive outcomes of the water hyacinth invasion was to spur on the need for basin management.

The invasion of the water hyacinth raised the need for effective collaboration among the riparian states. Despite the lack of a coordinating entity in the basin, Uganda, Tanzania and Kenya governments at all levels, as well as local communities, cooperated successfully to combat the waterweed. In 1995 a biological control, the weevil, a type of beetle, was introduced to control the proliferation of the water hyacinth. Dramatic declines in the weed were observed in subsequent years. Through the efforts of the riparian states, and the international community by means of the Lake Victoria Environmental Management Program (LVEMP), water hyacinth was reduced by approximately 78 per cent lake-wide (Kayombo \& Jorgensen, 2006). An 
important aspect to the success of the weevil was the involvement of local fisher communities in rearing and distributing weevils. While weevils were not expected to overcome the problem entirely, the combined effort with mechanical removal has successfully controlled the weed and its impacts on basin activities. However, there have been reports of resurgence in water hyacinth as a result of elevated pollution and nutrient loading from the catchments.

Notwithstanding the adverse effects the water hyacinth had on the lake, it ironically had the benefit of reducing fishing pressure. Kateregga and Sterner (2009) reported on average, catchability of fish, the extent to which a stock is susceptible to fishing, was reduced by 45,2 and 6 percent in Kenya, Tanzania and Uganda, respectively. The larger reduction observed in Kenya could be attributed to the high abundance of water hyacinth mats in this country's waters. The weed is also believed to have promoted fish diversity. There have been reports of the reappearance of certain species that had been declared extinct in the past 15 years (Kateregga \& Sterner, 2009). Since the decline of the weed, fish harvesting has again accelerated.

The shift from commodity-production management to an ecosystem management approach for Lake Victoria has revealed the importance of wetlands to lake sustainability. Wetlands anchor soils, catch silt and filter out pollutants, all of which can prevent eutrophication and further deterioration of water quality. Wetlands also provide for sustainable fishing, support a rich array of birds that inhabit the lake throughout the year and provide physical stability to the shores of the lake (Kairu, 2001).

About 75 per cent of the wetland area in the Lake Victoria basin has been significantly affected by human activity. Approximately 13 per cent is considered severely degraded (Kayombo \& Jorgensen, 2006). This could be attributable to the draining of wetlands for purposes of increased agricultural activity and increased demand for settlements. The 
sustainability of the lake therefore requires appropriate sustainable wetland management as an integral element in the protection of Lake Victoria water resources (Ntiba et al., 2001). This could include conservation and environmental protection and enhanced institutional cooperation and public participation (Kairu, 2001).

As one can observe, the challenges facing Lake Victoria are alarming. The projected population increase in riparian communities will inevitably exacerbate these issues. Effective management of Lake Victoria by all five countries within its basin is critical for its sustainability. A number of management systems have been established and have evolved in an attempt to respond to these issues.

\subsection{Lake Victoria Governance}

There has been a long history of trilateral cooperation in the management of Lake Victoria. There is evidence that the riparian countries began collaborating as early as the $1920 \mathrm{~s}$ in response to concerns over the region's fishery (NaFIRRI, 2009, p. 15). The extent of cooperation, however, has varied throughout the years, reaching its peak when political relations were collegial as illustrated by the creation of the East African Community to periods of inactivity during times of political instability. Over the past two decades, Uganda, Tanzania and Kenya, with the assistance of global partners, have been forging stronger partnerships to manage the lake's resources in a holistic fashion. The following discussion offers an overview of the historical and existing frameworks established to manage Lake Victoria. I suggest these evolving institutional structures have been established in parallel with the increased scientific understanding of the lake ecosystem. 


\section{History of Lake Victoria Management}

As early as the 1920 s, there were suspicions by the then ruling British authority that the fishery was showing signs of deterioration in some parts of Lake Victoria. A survey was commissioned in 1927-28 to confirm those suspicions and determine the extent of changes experienced by the fisheries. Among other observations, the survey concluded that there were signs of overfishing tilapia stocks (NaFIRRI, 2009, p. 15). Survey results indicated the need to establish an authority to collect fisheries statistics for management use and to establish a research base on the lake. This led to the creation of the East African Freshwater Fisheries Research Organization (EAFFRO) in 1947 with five scientists investigating only tilapia fisheries of Lake Victoria (Odada \& Olago, 2006; NaFIRRI, 2009). The EAFFRO was further strengthened by the birth of the East African Community (EAC) in 1967, which was established after the countries gained independence. The EAC became the regional coordinating mechanism to harmonize development and policies, including Lake Victoria management.

The EAFFRO collapsed in 1977 when the treaty that had established the East African Community was officially dissolved. The dissolution of the EAC was the result of Uganda's dictatorship regime and resulting political instability during that time (Interview, May 25, 2009, Jinja, Uganda). However, the need to have a coordinating body for Lake Victoria affairs remained. The Committee for Inland Fisheries of Africa (CIFA) was therefore utilized to coordinate aspects of Lake Victoria fisheries. The CIFA was established in 1971 under the auspices of the United Nations Food and Agriculture Organization (FAO). The CIFA was mandated to assist member countries with developing the scientific basis for regulatory and other management measures required for conservation and sustainability of inland fisheries resources. CIFA later established a subcommittee on Lake Victoria and facilitated regional collaboration for 
fisheries management. The Committee has not been as actively involved with Lake Victoria management since it fulfilled its mandate in the early 1990s. CIFA has since evolved to include aquaculture and is presently known as CIFAA - Committee for Inland Fisheries and Aquaculture of Africa.

\section{Present Governance Structures}

Over the past two decades, dramatic ecosystem-scale changes have occurred in the lake, which have necessitated further regional collaboration in Lake Victoria management. Through the resurrection of the EAC in 1984 and eventual signing of the Treaty for the Establishment of the East African Community in 1999, the three riparian countries designated the Lake Victoria Basin as an economic growth zone that must be developed sustainably (Kayombo \& Jorgensen, 2006). The EAC recognized a regional coordinating mechanism was required to address concerns regarding fish stocks and to effectively manage the fisheries resource. Efforts by the EAC, the three riparian countries, the FAO, European Union through the Lake Victoria Fisheries Research Project (LVFRP), World Bank and GEF through funding of the Lake Victoria Environmental Management Project (LVEMP) culminated with the creation of the Lake Victoria Fisheries Organization in 1994 (Klohn \& Andjelic, 2008; Kayombo \& Jorgensen, 2006). The EAC then established the Lake Victoria Basin Commission (LVBC) approximately a decade later to promote sustainable development of Lake Victoria resources through the adoption of a multisectoral, regional approach to management. The LVBC is the regional coordinating mechanism for the basin and has a much broader mandate than the LVFO. A discussion on these two transboundary entities as key components of the Lake Victoria management regime is provided later in the chapter. Complementing these Lake Victoria structures are national departments and agencies such as the Uganda Ministry of Water and Environment and its Directorate of Water 
Resources Management and the National Institute for Fisheries Resources Research Institute (NaFIRRI). These organizations have considerable roles in the management of the lake. However, given this paper's focus on collaborative management, the independent mandates of national governments are not addressed.

As cooperative agreements are important for effective transboundary water management, so is the Convention for the Establishment of the LVFO in maximizing commitment and meaningful participation by all three governments and other stakeholders in protecting Lake Victoria (Ntiba et al., 2001). The agreement formalizes the parties' recognition that management decisions undertaken by one party can significantly affect the portions of Lake Victoria lying within the territorial limits of another party. The Convention also highlights the role of science in the governance of lake fisheries by recognizing the "continuing need to increase the scientific understanding of Lake Victoria including its living resources, ecosystem and impact on those resources of climate, human population and settlement, non-indigenous wildlife and industrialization." Unlike the Canada-United States Great Lakes Water Quality Agreement (GLWQA), which is subject to periodic review every six years, the convention has not yet been reviewed since its signing in 1994 nor is there a provision in the convention that stipulates a review cycle.

The main function of the LVFO is to promote proper management and optimum utilization of Lake Victoria fishery resources by undertaking activities including but not limited to coordinating fisheries management for the conservation and use of other lake resources; collaborating with existing bodies and programs dealing with lake management; and disseminating information on Lake Victoria. The organization also has a considerable scientific 
role and functions to conduct research on existing and emerging causes of environmental degradation and to harmonize standards and data across all riparian countries.

The harmonization function of the LVFO is a critical factor for forging stronger relations among the policy and science communities in the region. Fragmented efforts with respect to research and monitoring practices can paint very different pictures of the state of the lake. Further, given the challenges of limited human and financial resources in the region, the LVFO can maximize research investments through harmonization efforts. The LVFO therefore attempts to coordinate initiatives to avoid duplication of efforts and to capitalize on limited financial and human resources.

The governing structure of the LVFO also reflects this commitment to science by establishing a process that allows science to be formally integrated into the policymaking process. The LVFO governance structure consists of a Council of Ministers (COM), a Policy Steering Committee, an Executive Committee, a Fisheries Management Committee, a Scientific Committee and National Working Groups. The organ that allows for scientists and policymakers to directly interact is the Executive Committee. This governing approach incorporates the positivist concept where science can help define policy priorities and policy objectives can guide research activities.

The Executive Committee of the Lake Victoria Fisheries Organization (LVFO) consists of the department heads responsible for fisheries management and fisheries research in all three riparian countries. It is within this organ where the confluence of fisheries policy and fisheries science occurs. Members deliberate on priority scientific and management fisheries issues such as the introduction of a new exotic species to recover fish stocks and the elimination of excess boats (Interview, May 25, 2009, Jinja, Uganda). The Executive Committee recommends priority 
issues to the Policy Committee for further deliberation, who subsequently makes

recommendations to the COM based on the advice received by the Executive Committee. The extent the COM accepts or disagrees with the advice of the Executive Committee is not apparent. However, policy decisions made by the COM are thought to be sound as a result of being informed by scientific and management advice.

While a proviso for the establishment of Beach Management Units (BMUs) is not in the Convention, they are a recent participatory innovation that will be formally integrated into the LVFO (LVFO, 2009). BMUs were introduced into the governing system by the national governments when they recognized that regulations were frequently being violated and a new mechanism was required to assist with implementation and enforcement. BMUs are the foundation of co-management in the region and establish a partnership arrangement in which the community of resources users, government and other stakeholders share the responsibility and authority for the management of the fishery (LVFO, 2009). BMUs instill a sense of ownership, responsibility and accountability over lake resources and have the added benefit of providing local fisher communities with an avenue to express their concerns and ideas to the LVFO. To date, 1,087 BMUs have been established in the lake basin (LVFO, 2009).

As the scientific understanding of the ecosystem evolved, revealing linkages between the various components of the lake and cross-sectoral anthropogenic activities, riparian states and international partners recognized the need for an integrated water management approach. In November of 2003, the EAC Council of Ministers, with the assistance of the GEF and other international donors, approved the Protocol for Sustainable Development of the Lake Victoria Basin - 'the Protocol'. This protocol formalized the parties' recognition that an integrated 
holistic approach for managing Lake Victoria is essential for the viability of the resource. It also provided for the creation of the Lake Victoria Basin Commission (LVBC).

Adopting an IWRM approach in the Lake Victoria region requires lake managers and scientists to shift their thinking toward 'sharing benefits' rather than 'sharing quantities of water' (Interview, June 11, 2009, Entebbe, Uganda). A senior official at the Directorate for Water Resources Management shared that national departments are now designing water resource plans that integrate issues across various sectors, broadening the focus to the region as a whole so the lake can be managed as one unit (Interview, June 11, 2009, Entebbe, Uganda). It has been reported that there has been some difficulty with instituting this new approach owing to the magnitude of the socio-economic challenges facing the region and the multiple players involved in the process. "But it's something that can be realized with time" (Interview, June 11, 2009, Entebbe, Uganda).

The LVBC is mandated to govern activities beyond the fisheries sector and encompass an integrated water resources management approach to Lake Victoria. Managing the lake basin as one unit also requires participation from Rwanda and Burundi in addition to the three riparian countries. While they may not border the lake, activities within these countries could have detrimental effects on the lake.

LVBC strives to achieve IWRM by strengthening science through harmonization and research initiatives, and enhancing stakeholder participation. The commission is expected to harmonize policies, laws, regulations and standards for Lake Victoria basin activities. The LVBC is also expected to promote coordinated research development including, issues of socioeconomic development and other natural resources that are beyond the fisheries sector. There is 
the added complexity of harmonizing activities across various sectors as policies related to land use management for example, are vastly different between countries.

The Protocol for Sustainable Development of the Lake Victoria Basin also sets out provisions for the LVBC to promote stakeholder participation in the sustainable development of natural resources, capacity building and institutional development. However, since the creation of the LVBC in 2004 to the time this research was conducted, there has been no formal communication and coordination channel that can enable a Civil Society Organization (CSO) to formally interact with the LVBC (National Consultative Meeting between Uganda CSOs and LVBC Proceedings, 2009). Given that public participation is deemed to be a critical component for the sustainable development of Lake Victoria, this "can only be meaningful if the capacity of [stakeholders, such as] CSOs networking in countries around Lake Victoria is well developed, and when [they] are recognized as valuable partners in development" (National Consultative Meeting between Uganda CSOs and LVBC Proceedings, 2009).

The Commission structure includes a Sectoral Council, Coordination Committee, Sectoral Committees and a Permanent Secretariat. The Secretariat carries out the main science functions for the LVBC. The Protocol stipulates the Secretariat create a regional database, promote information sharing and develop data exchange mechanisms. The Secretariat also facilitates and coordinates research on sustainable development including the Second Phase of the Lake Victoria Environmental Management Project that focuses on research, management and, social economic development of the basin. It has the added functions of harmonizing standards and data across the basin countries and disseminating information of LVBC activities to stakeholders and the international community. 
Given that the LVBC is a relatively new player in the Lake Victoria management regime its functions have only recently become fully operationalized. Since its inception, the LVBC has undertaken projects to address pollution and fisheries issues, as well as social matters within the basin such as HIV/AIDS response for mobile populations. When asked about the contribution and value of the LVBC to Lake Victoria basin management, interviewees expressed that it is too soon to tell.

The LVBC and LVFO are both entities of the East African Community (EAC) although it is unclear whether they report directly to the EAC. They differ in scope as the LVBC is mandated to govern activities beyond the fisheries sector and to adopt an integrated water resources management to Lake Victoria. As a specialized and autonomous institution of the EAC, LVFO programs and activities are in line with the Protocol for Sustainable Development of Lake Victoria (LVFO, 2009). Similarly, under 'the Protocol', the partner states are required to manage, develop and utilize fishery resources in accordance with the Convention Establishing the Lake Victoria Fisheries Organization. These provisions strive to harmonize fisheries efforts among the two organizations. However, should there be any inconsistent agreements relating to Lake Victoria, the Protocol "shall take precedence over any other existing agreements" (Article 48). Despite these provisions, it is recognized that there are some areas of intersection among the functions of the LVFO and LVBC that have caused an element of confusion among lake managers and scientists. There is a need to clearly delineate the roles and responsibilities of the commission.

\section{Challenges of Lake Victoria Governance}

Notwithstanding the advances made in Lake Victoria governance, there remain many challenges that can obstruct the sustainable management of basin resources. A common theme in the 
activities of both the LVFO and LVBC is the harmonization of standards and data as it is fundamental for all countries within the basin to work from the same canvas. The LVBC is tasked with the added challenge of coordinating the development of integrated strategies that cross multiple sectors. Although recent efforts strive for regional coordination, the fragmentation of efforts continues to be at issue in the region owing to institutional barriers within administrations.

Reaching consensus on baselines, management protocols and water quality standards is a challenging but necessary task. As illustrated in the previous chapter, essential to transboundary water management is the availability of adequate information to inform the development of policies. As harmonization and standardization of scientific methods and data collection continue to be an issue in the Lake Victoria region, policymakers are faced with disparate data sets for policy development. In these situations, the goal of establishing effective and sustainable policies cannot be realized. It has been observed, "even a uniform set of data that describes the state of the lake's water quality has not been assembled from the separate national data collection efforts" (Kayombo \& Jorgensen, 2006, p. 439).

It has been suggested that the greatest ongoing challenge in the management of Lake Victoria and its basin is the development of an integrated management plan (Ntiba et al., 2001). Sectoral approaches to water resources management that have dominated in the past are still prevailing. This has led to fragmented and uncoordinated development of the resource. Agriculture, livestock and forestry policies do not give particular attention to issues of transboundary water resource management despite the adverse effects they could have on the lake (Kayombo \& Jorgensen, 2006). Further, there are conflicts with other departments within the same administration. For example, a Lake Victoria manager revealed that there are conflicts 
between water managers and those in energy due to disputes over water release (Interview, June 11, 2009, Entebbe, Uganda). Until such time when integrated water resources management and a cross-sectoral approach is fully accepted and adopted within national government departments, the fragmentation of policies will continue. This is a pre-requisite for the eventual integration of sectoral activities across all riparian countries.

Over the past century, the riparian countries have collaborated on the management of commodity production, specifically for the sustainability of the lake's fisheries. With the evolution of scientific knowledge, management efforts are now shifting toward a more integrated and holistic approach for the basin. While there have been significant advancements in Lake Victoria management, there remain challenges that have delayed and in some cases obstructed the development of necessary policies in the region as with seasonal closures and policies for illegal fishing equipment. Some obstacles, as in the case of harmonization of standards and data collection methods, could be overcome by strengthening the connection between science and policy. In the next section of this paper, I examine the science-policy dynamic in the Lake Victoria management regime and examine the tools and mechanisms that have been adopted to integrate science into the policy process.

\subsection{The Science-Policy Interface}

The challenges of integrating science into the Lake Victoria management regime are characteristic of transboundary areas. The issues of disharmonized policies, standards and data collection methods and fragmentation of efforts within and between administrations are incessant and can be exacerbated in poorer areas like the Lake Victoria basin. Lake managers and scientists are recognizing that building a stronger science-policy interface can help address some of those challenges and narrow the gap. In the discussion that follows, this thesis shows that 
scientists and policymakers in the Lake Victoria Basin are beginning to adopt tools and mechanisms that can bridge the science-policy divide. These efforts parallel the instruments previously identified as important for transboundary water management. However, it is too early to evaluate if better integration of science is influencing policy outcomes given that the management regime is still very young.

It is appropriate to include a caveat with respect to the observations made in this section of the paper. An overview of the literature on the science-policy interface in environmental policy development was presented in Chapter Two. It showed that little research on the challenges of integrating science in policy processes has been done on transboundary water management regimes and specifically for Lake Victoria. As such, the following observations are predominately informed by interviews and in some instances, inferences drawn from general literature on Lake Victoria. It was previously indicated that the interviews were held with scientists, lake managers, academics, and NGOs residing in Uganda, therefore a Ugandan perspective dominates. Future research would benefit from a similar interview protocol with key individuals in Tanzania and Kenya. The literature was used as a point of reference to the extent possible in efforts to minimize a Uganda-centric perspective. These caveats noted, it remains that this thesis and these observations are making a unique contribution to our understanding of the science-policy interface and can serve as an early foundation for future work in this area.

It should also be noted that, in my opinion, the majority of scientists, researchers, policymakers and lake managers interviewed freely shared information and were relatively optimistic with the efforts made on Lake Victoria management to date. It was evident that civil servants employed to manage the lake are passionate and committed to its sustainability. However, it must also be highlighted that they are also under tremendous resource and political 
pressures. Interviewees recognized that there is no perfect system and progress is slow. Notwithstanding these challenges, these Lake Victoria custodians continue to work with whatever scientific and policy tools are available to protect this valuable resource.

Lake Victoria managers and researchers' interest in the science-policy interface is incipient. The science-policy gap has hitherto been unrecognized or seen as inconsequential by policy and decision-makers. The absence of literature on the topic as it relates to Lake Victoria supports this observation. There are now the beginnings of dialogue on the subject by both policy and science experts, with direction and guidance by the international community. The 'Lake Twinning Project' funded by the Global Environment Facility is one such example and has the purpose of developing a framework for collaboration on North American and African Great Lakes systems through enhanced science and policy linkages (Grover, 2009).

The Director at NaFIRRI admitted that management issues historically overshadowed the science. Relationships among scientists and lake managers were weak and communication was infrequent. In the past, "the director of a research department could take a long time without engaging the director of a management or policy department. And it would be a favour for one of the scientists to participate in a management meeting" (Dr. John Balirwa, Director, NaFIRRI, May 25, 2009, Jinja, Uganda). However, when lake managers realized the very apparent decline of the fish stocks, engaging scientists in the management process became more routine. Overwhelming scientific evidence coupled with real life situations has brought the two systems closer together (Dr. John Balirwa, Director, NaFIRRI, May 25, 2009, Jinja, Uganda).

A number of scientists also suggested that policymakers are now recognizing the importance of science but that is has been a long journey. A scientist at the Lake Victoria Fisheries Organization said: "to start with, it was difficult to convince policymakers but now of 
course...they want to be guided by science. And [policymakers rarely] make any decisions before they are properly informed by science" (Interview, May 26, 2009, Jinja, Uganda). Dr. John Balirwa, Director at NaFIRRI stated: "We [scientists] have worked hard to get the policy in line with realities. Sometimes it takes long but a lot has changed for the better" (Interview, May 25, 2009, Jinja, Uganda).

With this shift in information uptake, lines of communication have been opened allowing for increased information exchange and mutual appreciation of the challenges faced by the science and policy communities. More recently external stakeholders such as the public and non-government organizations have become important players in the dissemination of information. These trends are reflected in the instruments employed to narrow the divergence between science and policy.

\subsection{Integrating Science into the Lake Victoria Management Regime}

The cadre of Lake Victoria researchers and managers interviewed for this study were in agreement that strengthening the science-policy linkage would benefit the management regime. When asked to identify the means in which science has been integrated into the policy development process, interviewees revealed tools and mechanisms that parallel those identified in the discussion on transboundary water management including: adopting communication tools; establishing organizational structures; creating partnerships; investing in research, monitoring and reporting; and engaging public participants in the policy process.

\section{Communication Tools}

Lake Victoria researchers and managers have adopted a number of communication tools for better sharing of information. Managers noted that the inability of scientists to translate and 
package technical information into useable products is a hindrance in the policy development process. "[It is important to] transform scientific knowledge into practice so it can be of practical use to government and to people on the ground" (Interview, June 11, 2009, Entebbe, Uganda). Further, participants at the Twinning Workshop noted that science is usually conducted by researchers in a vacuum and is often ineffective in influencing resource management and policy decision. Organizational arrangements are needed to link science and policy and to provide a means to effectively translate the information into a language that makes it usable (United Nations University, 2008).

Boaz Keizire, previously worked extensively on fisheries issues in Uganda and highlighted the use of targeted communication as a mechanism to enhance information exchange between scientists and non-technical audiences (Interview, May 29, 2009, Kampala, Uganda). He shared that information on fisheries management was packaged into customized, userfriendly formats with key recommendations for different users such that it can be disseminated to various stakeholders by clusters and different groups and users. Targeted communication requires that scientists define the objectives of information dissemination upfront and to develop a strategy that can best achieve their purpose. This thinking complements the view of Karl Schaefer, Environment Canada, whereby consideration of the audience or users of the information is essential in the production, translation and dissemination of technical information.

Lake Victoria researchers and policymakers both participate in the production of communication materials. Information products such as internal and public reports, brochures and newsletters are disseminated to policy and science communities, as well as the general public as a means of education and outreach. Within the LVFO structure, the Secretariat has the function to summarize original scientific reports into language comprehensible by lake 
managers. The Secretariat also has the responsibility to guide fisheries management and prepares internal scientific reports to the Science Committee, who then introduces the material to the Executive Committee. Tabling these reports before policymakers can inform the prioritization of management issues for consideration by the Policy Committee. This is how science can influence policy (Interview, LVFO, May 26, 2009, Jinja, Uganda).

The Uganda National Environment Management Authority (NEMA) produces annual State of Environment (SOE) reports for public consumption. NEMA works with various environment-related Ministries to compile data to assess the progress of initiatives and to identify emerging issues. The SOE report also includes policy recommendations, which are considered by members of Parliament (Interview, NEMA, May 28, 2009, Kampala, Uganda). As technology becomes more advanced, NEMA is utilizing illustrative tools to convey issues of sedimentation for example. Images are generated to depict the effects of anthropogenic activities and are viewed as more effective as they can visually illustrate the adverse impacts. Policy and decision-makers are able to clearly see and understand the message being conveyed (Interview, NEMA, May 28, 2009, Kampala, Uganda).

As previously suggested, creating opportunities for information exchange is an important mechanism to enhance communication. This is also true in the Lake Victoria basin. There exist formal and informal mechanisms that allow for these exchanges to occur, both of which are effective and necessary for the development of strong management frameworks.

A number of interviewees were of the opinion that regular informal interactions among scientists and policymakers are effective for enhancing communication. They highlighted that these informal channels are becoming more prevalent in the region as science and policy 
communities are forging stronger relationships. "The more interactions, the more we both understand the issues" (Dr. John Balirwa, Director, NaFIRRI, May 25, 2009, Jinja, Uganda).

Tom Waako, Program Officer at the Nile Basin Initiative further noted: "informal interactions work here...we need to take opportunities during meetings, or send a personal email to open the lines of communication" (Interview, June 9, 2009, Kampala, Uganda). Taking advantage of these informal opportunities strengthens relationships and creates a trusting and enabling environment for staff. Kayombo and Jorgensen (2006) highlight that strong informal networks have been established between fisheries researchers that will provide a basis for future cooperation on technical issues. The extension of these networks to lake managers could further enhance lake management.

Formal information exchange opportunities such as workshops and training opportunities create a forum for scientists, policymakers and other interested stakeholders to convene and share information. Scientific presentations can enhance the understanding of ecosystem trends and emerging concerns among non-technical participants. The LVFO for example, organizes regional stakeholder meetings or workshops to inform stakeholders of scientific findings. The Council of Ministers accelerated the frequency of these meetings from five to three years - an indication that stakeholder consultation is being given some importance in the Lake Victoria management regime by political leaders. International partners can also assist with opening lines of communication as illustrated by the recent GEF investment into a twinning initiative between the North American Great Lakes Commissions and those in the Lake Victoria Basin.

\section{Organizational Arrangements}

Common to transboundary water management systems, including Lake Victoria, is the creation of organizational structures to strengthen the science-policy linkage. Institutional structures such 
as the Lake Victoria Fisheries Organization and national research institutes can influence the way science interfaces with policy. These organizations can be the basis for communication activity, building knowledge capacity and harmonizing research efforts, which are important for narrowing the gap between science and policy. Godber Tumushabe, Executive Director of Advocates Coalition for Development and Environment (ACODE) is of the opinion that "what is important is to see if the structure is there within which you can communicate your science" (Interview, May 29, 2009, Kampala, Uganda).

Within the Lake Victoria management regime, there exist a number of formal mechanisms and structures that provide a forum for scientific and policy priorities to converge. The LVFO's Executive Committee is one such example as the forum allows for fisheries research and management directors to interact and influence policy development. The LVFO also has the potential to strengthen the science-policy interface through harmonization efforts. Standardizing research projects, monitoring, environmental data collection and other scientific initiatives can alleviate technical issues related to inconsistent methodologies and results. The LVFO has been successful in standardizing policies across member countries related to fish catch slot size and fish net size. The LVFO has also harmonized fisheries regulations and codes of practice for fish handling and quality assurance, which has been suggested as the organization's greatest accomplishment (Ntiba et al., 2001). However, there are other instances where discordant policies have led to conflict. For example, arrests of Ugandans have been made in Kenyan waters as a result of discordant fishing gear policies. Those allowable in Uganda may not be permissible in Kenya and vice versa (Boaz Keizire, May 29, 2009, Kampala, Uganda). The LVBC also has a coordination and harmonization function. 
In addition to regional Commissions, national institutions also bridge the science-policy divide by conducting research and producing information that feeds into the decision support system (Gaster Kiyingi, formerly Communications Manager, NBI, May 21, 2009, Kampala, Uganda). In Uganda, this is primarily done through the Ministry of Water and Environment and the National Fisheries Resources Research Institute (NaFIRRI). The Directorate of Water Resources Management (DWRM) of the Ministry of Water and Environment is the organ primarily responsible for water research activity. This includes monitoring and assessment. The ministry is also responsible for coordinating the activities of the Water Policy Committee, mandated by the 1997 Uganda Water Act to advise the Minister for Water on policy goals and issues in the water and sanitation sector. The Committee "provides an opportunity for multiministerial discussions, and guidance and advice to policy and decision-makers" (Interview, June 11, 2009, Entebbe, Uganda). Members include senior officials such as the Executive Director of NEMA and the Permanent Secretary of the Ministry of Water and Environment. However, it was revealed during the interviews that the Committee has not been functioning over the last four years owing to the low profile given to water resources management and the lack of a clear understanding of the Water Policy Committee's role. Efforts are currently being made to resurrect this group, suggesting that water management is garnering greater attention by senior officials.

NaFIRRI is a semi-autonomous Public Agricultural Research Institute (PARI) of Uganda, established by the National Agricultural Research Act 2005. NaFIRRI is the main department in Uganda for fisheries research. Its role is to conduct basic and applied research on fisheries in addition to aquaculture. Its core functions include: generating knowledge and technology of strategic importance for the management, development and conservation of fisheries resources 
and water quality; development and management of fisheries research information and ensuring collaboration with stakeholders; planning, monitoring and evaluation all fisheries programs undertaken by the institute to ensure conformity with national research strategy; and participation in problem identification and prioritization of fisheries research demands for the national research agenda (NaFIRRI, 2009, p. 21). The outcomes from these activities inform policy development through the national development process for fisheries as well as through the activities of the LVFO.

There are of course other institutions with scientific and policy functions, including national management research departments in Kenya and Tanzania. Time and financial constraints did not allow for interviews to be conducted with scientists and policymakers in these two riparian countries and therefore limited the breadth of this study.

\section{Partnerships}

Partnerships are primarily established for collaborative research and can be cultivated within or among national departments - 'internal partnerships' - or can involve stakeholders outside of government such as academe and NGOs. Partnerships can be instrumental in facilitating scientific integration and policy uptake. As Waiswa Ayazika, Environmental Impact Assessment Coordinator at NEMA notes, the Uganda National Environment Management Authority does not work alone (Interview, June 1, 2009, Kampala, Uganda). Instead, it works with and depends on a number of other agencies. He offers the example of the Directorate of Water Resources Management (DWRM). This directorate is the lead agency in water and conducts sampling, monitoring and performs analyses. The DWRM is well equipped to fulfill these tasks. NEMA works with the Directorate to translate this technical information for NEMA activities such as the publication of the State of the Environment report. 
Collaboration between government and academe is also a common approach to generating scientific research and building knowledge capacity within the country. As Kayombo and Jorgensen (2006) observed, universities in each country provide the main technical capacity in fisheries research although analytical capabilities are also available in various government ministries concerned with land and agricultural management. Frank Kansiime, Professor at the Institute of Environment and Natural Resources at Makerere University indicated that the Institute has a loose partnership with the government (Interview, June 8, 2009, Kampala, Uganda). However, if requests are made to conduct specific studies to inform policy development, the institute would consider obliging. In the past, students and professors have conducted research that contributed to national policies, including the 1995 Uganda National Policy for the Conservation and Management of Wetland Resources.

Non-government organizations also partner with scientists to generate knowledge. ACODE Executive Director, Godber Tumushabe recognizes there are certain competencies that are not resident within his organization (Interview, May 29, 2009, Kampala, Uganda). At times, it is necessary to engage other experts to satisfy the requirements of the research initiative. In such cases NGOs may tactfully select technical experts that have the influential ability to move the policy process forward.

International partnerships also have the potential to enhance information exchange and understanding among scientists and policymakers from various jurisdictions. For example, as part of the twinning exercise, commissions explored science and policy linkages and identified the challenges with integrating science into policy processes. Through these initiatives, international science can be used to guide and assist regional and local policymakers and technical staff in managing their resource. A comparative analysis between the African and 
North American Great Lakes systems creates opportunities for lake managers and researchers in these distinct parts of the world to have a forum to share lessons learned and best practices to improve existing management practices.

\section{Investments in Research, Monitoring and Reporting}

Knowledge and understanding of the state of the water body is a pre-requisite for improved capacity to sensibly manage the resource (Klohn \& Andjelic, 2008). Investments in research, monitoring and reporting can help in this regard and allow for increased understanding of ecosystem changes and for the development of informed policies. Some lake scientists are observing a shift in national interest in research activities, reflected through increased funding for scientific research (Interview, May 25, 2009, Jinja, Uganda). This suggests national governments are recognizing the importance of scientific information for managing the lake.

The majority of the experts interviewed indicated research and monitoring as a key factor in the development of management policies and as a critical component in determining policy success. A critical component for any water policy or program is the ability to determine a baseline from which progress can be evaluated. Once established, monitoring data can then determine policy effectiveness by using the baseline as a reference point. Kairu (2001) suggests that a sustained monitoring program is essential to ensure that all impacts are examined over a relatively longer time frame. Long-term trends are more accurate and can reduce scientific uncertainty.

The main problem for Lake Victoria is the lack of data (Interview, March 26, 2009, Toronto, Ontario). The Lake Victoria Environmental Management Project (LVEMP) Phase I is an example of a research initiative that strived to enhance monitoring and reporting of lake pollution and water quality. The LVEMP I strengthened scientific capacity in the Lake Victoria region by establishing 56 monitoring stations to determine in-lake pollution concentrations. An 
additional 18 monitoring stations were established on rivers draining into the lake (Kayombo \& Jorgensen, 2006). Once data are collected, the problems can then be identified and options for solutions can be explored. However if data are unavailable, lake managers may not even realize there is a problem. A more detailed discussion on the LVEMP I, the outcomes of the project as well as the second phase of the project, LVEMP II are discussed toward the end of this chapter.

\section{Public Participation}

The design of Beach Management Units at various landing sites across all three countries not only establishes a mechanism for monitoring and enforcement of fisheries activities, but also provides a means for indigenous knowledge to inform policy development. In theory, BMUs provide a means for fishers to communicate their knowledge and experience to fisheries officers. These government representatives would then report back to department managers with this intelligence to inform policy development. A fisheries officer interviewed at one of the landing sites in Kampala shared that through the BMUs, the concerns of the fishing community are being heard and addressed. "There are procedures under the BMU [to] hold regular meetings...All the members, including the crew fishermen, the women, processors... all come with their concerns and they are given a platform to air their views and to make a decision" (Interview, June 9, 2009, Kampala, Uganda). Although there are challenges with the operations of BMUs, it is a tool employed by all three riparian states and one that has the potential to help bridge the sciencepolicy gap.

The tools and mechanisms employed by Lake Victoria researchers and managers are similar to those observed in transboundary water management generally. The extent that they are incorporated into management practices is dependent on various factors distinct to developing areas that include resource constraints and political interference. In the next section, 
I discuss some of the challenges that continue to face Lake Victoria managers and scientists when attempting to narrow the divide between science and policy.

\subsection{Challenges of Narrowing the Science-Policy Gap}

Notwithstanding the efforts made to strengthen the science-policy linkage in the Lake Victoria management regime, there exist difficulties with the development and application of these integration tools and mechanisms. Resource limitations are one obvious challenge that begets other issues such as knowledge gaps and the inadequacy of data. These constraints also increase dependency on the international community. And given the transboundary nature of the regime, issues of political interference can also influence the effectiveness of these tools.

\section{Human and Financial Resource Constraints}

Despite the claim that there has been increased funding allocated to research efforts, there are still concerns over fiscal constraints. "Finances limit a lot of the measures that could be taken" (Interview, May 26, 2009, Jinja, Uganda). While there is the potential for improving the state of the lake's ecosystem, it cannot be realized with the limited funds apportioned for Lake Victoria management. Two interviewees expressed frustration with the distribution of national budgets. Godber Tumushabe of ACODE commented, "[There is] discrepancy between what seems to be the recognition of the issues by politicians and the allocation of funding to address these issues" (Interview, May 29, 2009, Kampala, Uganda). Another interviewee believes that the governments recognize the importance of science in policy development but also admits that national budget allocations to support scientific studies make this questionable. Financial limitations are a central concern expressed by the majority of respondents, scientists and 
policymakers alike, suggesting that both communities recognize the importance of science for the management of the lake.

There were differing views on human capacity limitations in the management of Lake Victoria. A number of interviewees highlighted the well-qualified staff in the various institutes governing Lake Victoria and recognized their potential to make real progress. However, this is impeded by financial constraints. One respondent shared that, "those technical guys, they will tell you I have a masters degree with a post-graduate diploma in this and this and I studied in America...they have a lot of competence. [However, they] sit there [because they have] no budget to operationalize the activities of the position for which [they were] recruited. But [they] continue to receive a salary" (Interview, May 21, 2009, Kampala, Uganda). The respondent also noted, "it's not a lack of expertise, it is the lack of resources that allows the experts to work effectively." This was confirmed by a water scientist who shared, "all the scientists are pretty knowledgeable but they do not have a lot of experience with implementation" (Interview, June 11, 2009, Entebbe, Uganda). Conversely, a water manager noted that one-third of the positions are filled at the individual's organization and this affects policy development, "you have the structure but do not have the qualified personnel" (Interview, June 11, 2009, Entebbe, Uganda). The effect of 'brain drain' was also highlighted as a challenge in maintaining qualified staff within the region. Of course, this is experienced in all parts of the world and not only in the Lake Victoria region.

\section{Inadequate Monitoring and Data}

The challenges resulting from financial and human constraints include inadequate monitoring and data collection, both of which are necessary for policy development. A researcher expressed that policymakers want the scientists to given them a magic formula to solve the problem and to 
provide them with options to address the issue. Everyone, the fishers and managers, want targets but the scientists are unable to settle on a target because they do not have the science to support the targets (Interview, May 26, 2009, Jinja, Uganda). As previously emphasized, long-term monitoring data is critical to assess ecosystem changes and to evaluate the effectiveness of policies. Scarce data can hamper the evaluation of ecological changes in the context of aquatic food-web alterations, catchment disturbance and natural ecosystem variability (Verschuren et al., 2001). Further, there are also risks of gathering erroneous data as a result of inexperience on the part of the researcher. Data relevancy is therefore a concern in the region. Identifying the correct water parameters for monitoring can be a challenge for Lake Victoria management (Interview, March 26,2009, Toronto, Canada). A number of scientists noted that lack of personnel and equipment limit data collection efforts.

\section{Dependency on International Community}

Although questions of donor dependence were not asked during the interviews, when the issue of international support did arise, contradictory perspectives from the respondents were received. The interviewees who spoke on this topic were of the mind that international funding has and will continue to play a role in Lake Victoria management. Seeing as infrastructure for fisheries and water quality research is expensive, there will be a continuation of capabilities dependent on further external investments (Kayombo \& Jorgensen, 2006). The Director of NaFIRRI acknowledged, "[during] the last 5 years, the region has been lucky to be enjoying support from the European Union for all these [fisheries] activities. So increasingly, it is seen as possible to have transboundary issues resolved harmoniously" (Interview, May 25, 2009, Jinja, Uganda).

Conversely, another expert expressed concern over the countries' dependency on donors and suggested that this can affect policy outcomes. The respondent offered the following 
example. When a project ends, nothing gets done until the next project is funded because national governments have not taken responsibility (Interview, May 26, 2009, Jinja, Uganda). The interviewee further suggested the security of international funds for Lake Victoria projects allows national governments to allocate funds for other projects, which further detracts national responsibility over Lake Victoria. An expert from an international funding organization noted that the sustainability of financing continues to be a challenge and therefore may prolong the dependency on donors (Interview, March 26, 2009, Toronto, Canada).

\section{Political Interference}

The fact that Lake Victoria is a transboundary resource and bordered by developing countries makes it prone to political instability and interference. As one interviewee noted, "politics have crippled the ability of policymakers to make any meaningful decisions" in the region (Interview, May 29, 2009, Kampala, Uganda). This policy expert further stated: "in this country [Uganda], we implement good politics. We do not implement good policies," implying that politics dominate policy agendas. Another respondent commented that consultants hired to conduct work on Lake Victoria are disappointed with the slow progress and enormous political and socioeconomic barriers (Interview, May 26, 2009, Jinja, Uganda).

Historical evidence illustrates how political interference has disrupted scientific efforts. Throughout the early 1950-60s, Ugandans explored the aquaculture industry as an alternative option for open-water fishing. The political instability arising from the Amin dictatorship caused the abandonment of such efforts (NaFIRRI, 2009, p. 10). One interviewee also shared an experience whereby the Minister responsible for energy was reported to have influenced the amount of water released for energy production (Interview, June 11, 2009, Entebbe, Uganda). Further, policies previously established on issues of water release continue to be implemented in 
the current management regime, including 'The Agreed Curve'. This policy was arrived at in 1954 between Egypt and the British colonial administration in Uganda to govern water release (Salman \& Salman, 2009). It is recognized that dramatic ecological changes have occurred in the lake since the policy was established yet it has not been adapted to address those changes. Recently, however, the Lake Victoria Basin countries have formally requested the Lake Victoria Basin Commission review the Agreed Curve policy and to consider developing a new policy that is reflective of the current environment (Interview, June 11, 2009, Entebbe, Uganda).

Political influence is beyond the control of lake scientists and policymakers. In situations where politics dominate the policy stage, science may be overlooked. In such cases, Godber Tumushabe of ACODE suggests the mobilization of the public is one avenue in which political motives can be averted (Interview, May 29, 2009, Kampala, Uganda). For example, in 2007, ACODE and other organizations mobilized the public and other groups and mounted a very powerful campaign to protest the Ugandan President's plans to convert parts of the Mabira Forest Reserve into a sugar cane plantation. Mabira Forest is linked to the ecological integrity of Lake Victoria and alterations in land use could potentially have adverse implications on the lake and its basin. The campaign proved successful and was the first time, in the history of the country where an environmental demonstration forced a political leader to abandon his idea.

While strengthening the science and policy connection is important in the development of sound policies for the protection of the Lake Victoria basin, there exist factors that are beyond the control of lake scientists and managers that prevent this from coming to fruition. In such cases, it may be necessary to mobilize partners outside of government to move the policy agenda forward. Further, until such time when national governments take greater responsibility for the 
management of the shared resource, they will continue to be susceptible to inadequacies with their scientific capacity as a result of their dependence on donors and international partners.

\subsection{Evidence of Scientific Influence on Policy and Project Outcomes}

It appears that major steps have been taken in an attempt to build a stronger relationship between science and policy in the Lake Victoria management regime. While these efforts allow science to become better integrated into policy processes, the influence of science on policy outcomes is not yet clear. Given this ambiguity and the absence of literature on the science-policy dynamic in Lake Victoria, I was interested in learning about existing policies that interviewees considered both scientifically sound and effective. I learned that there are few Lake Victoria policies that meet both criteria.

When asked to identify an evidence-based and effective management policy for Lake Victoria, the majority of interviewees responded with hesitation. The term 'effective' was not defined for the candidates rather the interpretation of what is deemed effective was left to the respondents. The majority of interviewees defined 'effective' to mean measurable improvements in lake quality. With the exception of one out of the 15 interviewed on the subject matter, respondents were unable to quickly produce concrete examples of effective policies, if at all. In fact, out of the thirteen interviewees asked, seven were unable to identify any evidence-based policies that have proven successful.

With respect to fisheries, an interviewee with extensive water policy experience indicated, "fisheries policy has not been effective at all despite the science. The practice has not been sufficiently managed so you tend to find depleted fisheries resources" (Interview, June 11, 2009, Entebbe, Uganda). Another respondent emphasized that the policies are in place and the issue facing lake managers is operationalization. Additionally, Tom Waako of NBI, noted that 
the "success of policies sometimes takes time to judge. By the time you realize there are benefits, maybe we are talking about 10-20 years down the line" (Interview, June 9, 2009, Kampala, Uganda).

Some experts were optimistic and believed there are opportunities now to develop scientifically sound and effective policies. Such an opportunity lies within the East African Community protocol on the environment. The protocol includes an annex outlining the provisions for environmental impact assessments for Lake Victoria management. Nonetheless, when given sufficient time, a few respondents identified some common policies that they believed are both evidence-based and effective. These include sustainable fisheries policies on Nile perch slot size and gill net size. Further, the eradication of the water hyacinth was reported as a success by four interviewees. Two of those respondents also credited the efforts of the Lake Victoria Environment Management Project as helping to manage and control the weed. Lastly, Uganda's wetland policy was highlighted as scientifically sound and a policy that has resulted in improvements in the Lake Victoria basin. While the majority of respondents had difficulty identifying effective and evidence-based policies, the LVEMP was highlighted by a number of experts as being key to developing scientific capacity in the region that will help bridge the science and policy gap in the future.

\section{Lake Victoria Management - Projects of Influence}

During the 1990s, two projects financed by the European Union, World Bank and GEF shaped the governance and management of Lake Victoria. These projects were the Lake Victoria Fisheries Research Project (LVFRP) and Lake Victoria Environmental Management Programme (LVEMP). Both projects were designed to enhance scientific capacity for the region with the 
objective to better inform policy development. It is therefore important to highlight the purpose of the projects and the outcomes of the initiatives.

In 1997, the Lake Victoria Fisheries Research Project was established to assist the LVFO in developing a framework for the management of the lake's fisheries. At that time, countries were beginning to coordinate responses to fisheries management however a coordinated action plan had not yet been developed. Knowledge deficits were the impetus for this research project and activities were designed to carry out lake-wide research including stock assessments to fill the void, with the ultimate objective to inform the development of a coordinated plan. Through these efforts, information on fish stock, fish speciation, market surveys and species abundance were made available.

The LVFRP also rehabilitated and constructed research vessels and equipped research institutes in order for the LVFO to have the infrastructure and equipment in place to carry out research activities successfully. The LVFRP further investigated socio-economic issues related to the lake and its fisheries (Kayombo \& Jorgensen, 2006).

The Lake Victoria Environmental Management Project is a dual-phased initiative designed as the first comprehensive program for the management of the Lake Victoria basin. The first phase was initiated in 1992, followed by a Tripartite Agreement in 1994 with full implementation during the years 1997-2005. The impetus for LVEMP was derived from the National Environmental Action Plans of the three riparian countries. Each plan identified that Lake Victoria management required urgent attention and needed a coordinated, regional approach to ensure its sustainability.

LVEMP Phase I was a US $\$ 80$ million project funded by the GEF, World Bank, the riparian states and several donors. It was established to manage the lake from a regional 
perspective. The LVEMP was designed to address the main underlying features of declining biodiversity, oxygen depletion in the lake and reduced water quality. The LVEMP's three main objectives were to:

(1) Maximize sustainable benefits to riparian communities using basin resources;

(2) Conserve biodiversity and genetic resources; and

(3) Harmonize national management programs.

To achieve these objectives, LVEMP would: improve fisheries management and research; manage and control water hyacinth; manage and monitor lake pollution and water quality; manage land use and wetlands in the catchment; and support institutions for lake-wide research and management and pollution disaster contingency planning (Klohn \& Andjelic, 2008; Kayombo \& Jorgensen, 2006). A number of activities were initiated that could be identified as mechanisms to bridge the science-policy gap. These included: harmonization of rules, regulations and standards; facilitating information exchange of information and experience among the countries through workshops and training events; and maintaining communications with donors and international centres of excellence in research and training.

The LVEMP has been reported as being successful in helping riparian states achieve strong intergovernmental harmonization (GEF, 2009, p. 12). The project allowed the states to build the institutional, human and knowledge capacity to effectively address the main concerns in the basin. Through LVEMP I, it was reported that baseline and trend data have been made possible with the establishment of a total of 74 monitoring stations in the basin (Kayombo \& Jorgensen, 2006). Stakeholders in the three countries have further learned to work together through regular meetings, field visits, workshops and conferences (Orach-Meza \& Okurut, 2005). The LVEMP was also instrumental in the development of the LVFO and LVBC. "Although efforts to promote greater collaboration on fisheries management date back as far as 
1928 , these efforts never managed to achieve a strong intergovernmental harmonization among the riparian countries. Through the support of the LVEMP, countries were able to work together to design and initiate the LVFO" (GEF, 2009, p. 12). LVEMP I has been referred to as the first step in a long process that would lead to overall improvement in sustainable use of the natural resources in the basin (Orach-Meza\& Okurut, 2005). When asked about the success of LVEMP, a number of interviewees applauded the efforts of GEF and riparian countries and noted the positive impacts the project has had in the region. A respondent highlighted that the "project was influential in setting up the conversations and strengthening them" (Interview, March 26, 2009, Toronto, Canada). Through LVEMP, the management of Lake Victoria is moving toward an integrated water resources management approach.

Although there has been much praise over LVEMP I, one interviewee suggested that perhaps it was not as successful as it seems and "that is why they are launching the second phase, to close the gaps because I do not think we have achieved really much reductions in pollution" (Interview, June 1, 2009, Kampala, Uganda). The World Bank Independent Evaluation Group's (IEG) evaluation of the project in 2006 supports this claim. The IEG revealed that limited achievements were made in research on improving water quality and pollution. There were also a number of weaknesses with the design of the project, which limited its overall potential. The IEG observed the various components of LVEMP were poorly linked and lacked well-defined objectives. For example, design of the fisheries management components and the wetlands management component did not reflect the importance of wetlands in fish production (Barghouti, 2006, p. 6), thus questioning the efficacy of harmonization efforts. The efforts to sustain scientific capacity in the countries would not generate sufficient commitment and capacity for the next phase because the work did not provide a comprehensive picture of the lake's conditions 
and related policy responses. Although there were reports of enhanced harmonized efforts within the region, the IEG observed there was weak coordination among the specialized agencies implementing the assigned components or subcomponents. This was compounded by limited donor coordination of support to country-level activities (Barghouti, 2006, p. 25)

An overarching deficiency with the project was the lack of consideration for the use and application of research results. The IEG highlighted "the design did not support efforts to translate findings of scientific studies into practical policy and development plans (Barghouti, 2006, p. vii). As previously noted by Karl Schaefer of Environment Canada one of the fundamental reasons for the gap between science and policy is the lack of thought on the utility of the science: What is the science produced for? How will the results help inform policy or program development? (Interview, March 25, 2009, Toronto, Canada). The IEG states: "The weak link between scientific activities and policy planning reduced the efficacy of the project; the scientific findings could be useful only if adopted by the stakeholders at all levels, including policymakers, communities surrounding the lake shores, local institutions, civil societies, and others" (Barghouti, 2006, p. 9). "There were shortcomings in communication, data storage, and dissemination services, which all undermined the value of all the scientific components" (Barghouti, 2006, p. 10). This is another example of the ineffective application of good science and further support for policy input in the design of scientific projects to ensure their utility.

The second phase of the LVEMP recently received approval for implementation. LVEMP II extends participation to Rwanda and Burundi with the objective of formulating a more holistic solution to address the ecological challenges facing Lake Victoria including the resurgence of water hyacinth and increasing pollution levels (GEF, 2009, p. 13). LVEMP II 
applies the lessons learned from LVEMP I in the design of the project. LVEMP II therefore proposes to address some of the key issues identified in the first phase including:

1) Strengthening governance of water and fisheries resources;

2) Investing in pollution control and prevention measures;

3) Raising public awareness and participation; and

4) Project coordination and management.

Although there were weakness in the design of the LVEMP I, investments have resulted in substantial improvements in the lake as illustrated by the eradication of the water hyacinth and fisheries management that would otherwise not be realized. LVEMP II has the potential to build on these successes by strengthening the future governance of Lake Victoria. Identifying additional science and policy linkages and continuing to cultivate stronger relations among the science and policy communities will contribute to the success of the program.

\subsection{The Future of Lake Victoria Management}

Lake Victoria has undergone dramatic ecological changes over the past few decades resulting from the introduction of an invasive species and increased pollution owing to industrial and agricultural activities and urban development. It is expected these pressures will be exacerbated in the future as a result of emerging issues including impacts from climate change and with increased demands from growing populations and economies within the region and in countries in the Nile basin. Non-point sources and atmospheric deposition are also surfacing as problematic. Further, fishing pressures are expected to continue as one of the most challenging issues facing lake managers and scientists.

The magnitude of the challenges that lay ahead is enormous. National governments are recognizing the urgency for effective transboundary management as a means of sustaining the lake and its resources for current and future generations. Industry and local communities are also 
beginning to realize their role in the protection of the lake and its resources. This is particularly evident with respect to the impending collapse of the fisheries. The Lake Victoria management regime is therefore evolving to one that is more integrated, holistic and participatory.

The key actors in the Lake Victoria management regime have made significant strides to forge a stronger connection between the science and policy communities. It has taken much time to arrive at this point, but scientists and lake managers are welcoming this paradigm shift. Closing the gap between science and policy allows for increased communication and information flow between scientists and policymakers, resulting in improved understanding and appreciation of objectives and processes for the development of informed policies. The extent that science can influence policy outcomes has yet to be seen as the Lake Victoria management regime has only recently emerged. However, if scientists and policymakers continue with these practices, the management system has the potential to produce similar outcomes as their North American counterparts: a regime that is internationally and domestically recognized as relevant, scientifically credible, and successfully trying to reconcile competing demands on an extraordinarily valuable resource. 


\section{Chapter 6: Conclusions and Recommendations}

Policymakers have the challenge of maneuvering through the ecological, socio-economic and political pressures, characteristic of environmental issues. This convoluted journey requires that policymakers are well-informed as their decision at particular junctures in the process will shape the direction and outcome of policies. Throughout this paper, it is illustrated that the inclusion of science in the policymaking process is one way in which policymakers can be better informed.

The integration of science can occur at any point in the policy development process and how this happens, and the way this happens can determine the degree of influence science can have on policy outcomes. This thesis concludes it is most optimal for policy to interface science at the beginning stages of the process when policy objectives are being defined. Thus, adopting a logical positivist approach for environmental policy development can allow for this to occur. While this paper argues that scientific integration in the policy process is a determinant for policy effectiveness, it is recognized it is not the sole factor.

The science-policy interface can be weakened for a variety of reasons. Common to the environmental policy process is the challenge of ineffective communication among the science and policy communities. Communication deficiencies can give rise to other factors that widen the gap such as the lack of understanding of objectives and processes. The literature and interviews also suggest that the causes for the divergence are context specific, as in the case of transboundary water management generally and Lake Victoria specifically. The challenges experienced in these areas could be exacerbated owing to the complexities of transboundary management that include differences in cultures, languages and national management approaches. Management regimes in transboundary areas are also required to give due consideration to political sensitivities among riparian states. 
There is an abundance of literature that confirms the divergence between science and policy, concluding that there is a need to forge stronger relations among the science and policy communities. This thesis identified tools and mechanisms that have the potential to narrow the gap and concludes that the means to strengthen the connection between science and policy are similar regardless of the context. Instruments that minimize the gap within governments are also observed in situations of multilateral cooperation. Tools and mechanisms that open and sustain communication channels between scientists and policymakers are critical to the development of informed policies. Further, instruments that enhance the translation of technical information into usable, comprehensible formats are essential.

Communication tools can be formalized as in the case of establishing 'knowledge brokers' within institutions and organizing workshops and conferences. However, informal exchanges between scientists and policymakers are deemed more effective by the interviewees and as revealed in the literature, as these interactions can also cultivate stronger relationships. Public participation is also observed to be a critical tool for strengthening the science-policy interface. Non-government participants have the ability to build trust between the government and its constituents by effectively translating and communicating policy and science objectives. Adopting mechanisms that encourage stakeholder participation also allows indigenous knowledge to be considered in the development of policies that would otherwise be absent.

Establishing strong organizational structures and capacities is an effective albeit more difficult tool to implement. Strong organizations can enhance the interface between science and policy by creating forums for information exchange and interaction among scientists and policymakers. The impetus to create these institutional structures rests on acting when opportunities arise, promoting shifts in existing paradigms, and significant top-level commitment 
and leadership as illustrated by the creation of the Science and Technology Branch in Environment Canada and the Lake Victoria Fisheries Organization. While the creation of empowered organizations can be an effective tool for cultivating stronger connections between the science and policy communities, they need strong political commitment, long timeframes to operate and significant resources. The adoption of other tools and mechanisms such as those previously mentioned could be the necessary incremental steps leading to the desired paradigm shift.

Forging stronger connections between the science and policy realms is particularly critical and challenging in transboundary water areas. International waters are increasingly under pressure owing to rapid growth of populations and economies in riparian communities and increased competing demands among the countries. There are also emerging issues such as climate change that will have inevitable consequences. Designing effective transboundary management regimes is therefore critical to the sustainability of these shared resources. Strengthening the role of science in transboundary management regimes has the potential to improve relations between countries, leading to more collaborative undertakings and effective management approaches. As demonstrated by the North American Great Lakes regime, science can also enhance the credibility of the management system and cultivate more robust relationships. However, as illustrated by the discussion on the Great Lakes, transboundary areas suffer from the effects of the science-policy divergence as well. It is essential for lake managers and scientists to continue building the necessary tools to mitigate these challenges. In areas where the development of management systems is incipient, there are opportunities for creating stronger linkages between science and policy in the design of the regime. 
East Africa's Lake Victoria is an area that is receiving greater international attention. The lake and its resources serve a multitude of purposes within and beyond the region. The increasing demands on the lake require a strong management system to ensure the lake is sustainable for current and future generations. The Lake Victoria regime is evolving, with recent investments leading to the creation of transboundary commissions to govern lake activities. These commissions have a science function and allow for the confluence of scientific and management priorities to occur. Lake managers and scientists are observing that the sciencepolicy connection is becoming stronger through these activities and the adoption of tools that narrow the science and policy divide. However, management efforts continue to suffer from disaggregated efforts and discordant policies and standards. Further, given that the riparian countries are among the poorest in the world, they are increasingly dependent on international partners and are consequently susceptible to knowledge gaps as a result of project cycles.

While the observations and conclusions from this study reveal the intricacies of the science-policy connection in environmental policy processes, particularly in the forum of transboundary water management, exploring other transboundary water management models could enhance this research. Further, an area that requires greater attention and focus in future research on the Lake Victoria management regime is the role and progress of the Lake Victoria Basin Commission. Due to time and resource constraints, I was unable to delve into this area that I believe is critical for the future of Lake Victoria management. Nonetheless, to enhance the science-policy connection in transboundary water management, this thesis offers the following four recommendations to water managers, scientists and decision-makers: 
1. Address the challenges of ineffective communication through the adoption of tools and mechanisms that could enhance communication between scientists and policymakers. Creating formal and informal information exchange opportunities are important for communicating and translating scientific and management priorities and have the added benefit of cultivating stronger relationships among the science and policy communities. Communication tools are generally easier to implement and can contribute to the paradigm shifts necessary for more permanent institutional changes.

2. Encourage 'in-reaching' within national governments and organizations with eventual broadening to neighbouring jurisdictions. As management regimes are moving toward an integrated water resources management approach, implications from activities that are untraditional to the environmental realm will influence the success of these regimes. Engaging and educating partners in transportation, land use and agriculture departments for example could lead to the identification of synergies and discordances with existing and future policies and programs.

3. Continue with public outreach and participatory efforts in developing transboundary water management policies and programs. In some instances, enhance stakeholder participation by adopting mechanisms into institutional processes that allow for this to occur. Non-government participants can introduce indigenous knowledge into the policy process and also have the ability to facilitate policy uptake and implementation. They can also assist governments with the translation of science and policy objectives. 
4. Incorporate an evaluation component to the policy development process to ensure policies remain current and effective. Establishing regional monitoring networks is essential for determining policy success. Failing to evaluate policies risks ineffective and in some cases, erroneous policies that could have adverse implications on the shared resource.

The literature and interviews reveal that water managers and scientists recognize the value and need to strengthen the science-policy interface, particularly in transboundary water areas where the divergence can be exacerbated. Adopting a positivist approach to transboundary water management promotes regular and frequent scientific integration into policy processes, which can enhance policy credibility and contribute to policy effectiveness. There are a variety of tools and mechanisms to strengthen this connection, some of which can be easily adopted and implemented whereas others require much longer timeframes and top-level commitment. This thesis reveals that Lake Victoria managers and scientists are beginning to adopt these instruments and are narrowing the divide between science and policy. Continuing with these efforts has the potential to lead to more effective policies and management practices for the region. 


\section{REFERENCES}

Anderson, David. (2007, February 28). Lecture: Linking science to public policy. University of Guelph.

ANPED. (May 2006). Industry and progress: From sustained growth to sustainable production and consumption. ANPED Statement for UN Commission on Sustainable Development, $14^{\text {th }}$ Session, United Nations, New York. Retrieved March 27, 2010 from, http:/www.spac.anped.org/documents/ANPEDstatementonindustrialdevelopmentver. 3 000.doc.

Anukularmphai, A. Integrated water resources management. Asian Development Bank. Retrieved October 4, 2009 from, http://www.adb.org/water/narbo/2004/TrainingProgram/presentation-concept-IWRM.pdf.

Appelgren, B. \& Klohn, W. (1997). Management of transboundary water resources for water security: principles, approaches and State practice. Natural Resources Forum, 21(2):91100.

Armitage, D., Berkes, F. \& Doubleday, N. (2007). Adaptive co-Management. Vancouver (B.C.): UBC Press.

Asmal, Kader. (1998). Water as a metaphor for governance: issues in the management of water resources in Africa. Water Policy, 1:95-101.

Australian Government. Connected water: Integrated water resources management. Retrieved October 4, 2009 from Australian Government website, http://www.connectedwater.gov.au/water policy/integrated mgt.html.

Awange, J. \& Ong'ang'a, O. (2006). Lake Victoria: Ecology, resources, environment. Netherlands: Springer.

Balirwa, J. (2007). Ecological, environmental and socioeconomic aspects of the Lake Victoria's introduced Nile perch fishery in relation to the native fisheries and the species culture potential: lessons learned. African Journal of Ecology, 45:120-129.

Barghouti, S. (2006). An independent evaluation of the World Bank's support of regional programs: Case study of the Lake Victoria environmental management project. The World Bank Independent Evaluation Group. Washington, D.C.: World Bank Publications.

Bernauer, T. (2002). Explaining success and failure in international river management. Aquatic Science, 64:1-19. 
Bihamiriza, B. (2007). Legal and institutional frameworks for public and stakeholder involvement, case study: Lake Tanganyika Convention. Presentation at the African Regional Workshop, Public Participation in International Waters Management. Retrieved March 27, 2010 from, http://www.iwlearn.net/publications/ll/legal-and-institutionalframeworks-for-public-and-stakeholder-involvement-case-study-lake-tanganyikaconvention-bihamariza.

Bocking, Stephen. 2006. Nature's expert: Science, politics and the environment. New Jersey: Rutgers University Press.

Bormann, B.T., Martin, J.R., Wagner, F.H., Wood, G., Alegria, J., Cunningham, P.G., Brookes, M.H., Friesema, P., Berg, J. \& Henshaw, J. (1999). Adaptive management in: N.C. Johnson, A.J. Malk, W. Sexton, \& R. Szaro (Eds.) Ecological Stewardship: A common reference for ecosystem management (p. 505-534). Amsterdam: Elsevier.

Borre, L., Barker, D. \& Duker, L. (2001). Institutional arrangements for managing the great lakes of the world: Results of a workshop on implementing the watershed approach. Lakes \& Reservoirs: Research and Management, 6:199-209.

Botts, L. \& Muldoon, P. (2005). Evolution of the Great Lakes Water Quality Agreement. East Lansing (MI): Michigan State University Press.

Bradshaw, G.A. \& Borchers, J.G. (2000). Uncertainty as information: narrowing the sciencepolicy gap. Conservation Ecology, 4(1). Retrieved from April 22, 2009, http://www.ecologyandsociety.org/vol4/iss1/art 71.

Bremner, J. \& Zuehlke, E. (2009). Integrating population, health and environment in Uganda. Retrieved May 5, 2010 from Population Reference Bureau website, http://www.prb.org/pdf09/phe-uganda.pdf.

British Columbia Ministry of Forest and Range. (n.d.). Defining adaptive management. Retrieved May 5, 2010 from, http://www.for.gov.bc.ca/HFP/AMHOME/Admin/index.htm.

Brown, M.B. (2009). Science in Democracy: Expertise, Institutions, and Representation. Cambridge (Massachusetts): MIT Press.

Cahn, M. Linking science to decision-making in environmental policy: Bridging the disciplinary gap (exploratory essay). Retrieved October 18, 2009 from MIT Press (forthcoming), http://www.csun.edu/ cahn/rulemaking.html.

Centre for River Basin Administration, Delft University of Technology. (2005). Transboundary river basin management: State-of-the-art review on transboundary regimes and information management in the context of adaptive management. (NeWater Report Series, No. 10). 
Climategate 'gravy train' Professor at centre of international row over 'faked' global warming data received millions from government bodies, the EU, quangos and Nato. (2009, December 6). The Daily Telegraph, Page: 012.

Conca, K. (2006). Governing water: Contentious transnational politics and global institution building. Cambridge (MA): MIT Press.

Convention for the Establishment of the Lake Victoria Fisheries Organization. (June 30, 1994). Retrieved from the Lake Victoria Fisheries Organization website, http://www.lvfo.org/downloads/THE CONVENTION FOR THE ESTABLISHMENT OF THE LVFO FINAL EDIT.pdf.

Coyne, A. (2010, January 7). The truth is out there. Somewhere. How does one distinguish between genuine authority and received wisdom? Macleans. Retrieved from, http://www2.macleans.ca/2010/01/07/the-truth-is-out-there-somewhere/3/.

Creswell, J.W. (1998). Qualitative inquire and research design: Choosing among five traditions. Thousand Oaks (CA): Sage.

Dale, A. (2001). At the edge: Sustainable development in the $21^{\text {st }}$ century. Vancouver (B.C.): UBC Press.

Davies, P. (February 2004). Is evidence-based government possible? Presentation at the $4^{\text {th }}$ Annual Campbell Collaboration Colloquium, Washington D.C. Retrieved March 27, 2010 from, http:/www.nationalschool.gov.uk/policyhub/downloads/JerryLeeLecture1202041.pdf.

Duda, A. (2002). Monitoring and evaluation indicators for GEF international water projects. Washington (D.C.): Global Environment Facility.

East African Community. (2003). Protocol for Sustainable Development of Lake Victoria Basin. Retrieved from the Lake Victoria Basin Commission website, http://www.lvbcom.org/index.php?option=com_docman\&Itemid=102.

Encyclopedia Britannica. Definition of 'science'. Retrieved March 27, 2010 from, http:/www.britannica.com/EBchecked/topic/528756/science.

Environment Canada and U.S. EPA. State of the Lakes Ecosystem Conference. (n.d.). Retrieved March 8, 2010 from Binational.net website, http://binational.net/solec/intro e.html.

Finger, M., Tamiotti, L. \& Allouche, J., eds. (2006). The multigovernance of water: Four case studies. Albany (NY): State University of New York. Press.

Food and Agriculture Organization (FAO). (2006). The state of world fisheries and aquaculture. Retrieved from the FAO website, http:/www. fao.org/docrep/009/a0699e/A0699E05.htm. 
Fraser, D., Gaydos, J., Karlsen, E. \& Rylko, M. (2006). Collaborative science, policy development and program implementation in the transboundary George Basin/Puget Sound ecosystem. Environmental Monitoring and Assessment, 113:49-69.

Gaden, M. \& Krueger, C. (2010). Chapter 7: Multi-jurisdictional governance of the Great Lakes fishery: Can a nonbinding agreement work? in Woodrow Wilson International Center for Scholars, Canada Institute, Environmental governance on the $49^{\text {th }}$ parallel: New century, new approaches (p. 99-114). Retrieved from http://wilsoncenter.org/topics/pubs/IJC\%20FINAL1.pdf.

Gerlak, A. (2004). One basin at a time: The Global Environment Facility and governance of transboundary waters. Global Environmental Politics, 4:4, 108-141.

Global Environment Facility. (2009). From ridge to reef: Water, environment, and community Security - GEF action on transboundary water resources. Retrieved from the Global Environment Facility website, http://www.gefweb.org/uploadedfiles/publications/gef ridgetoreef cra lores.pdf.

Global Environment Facility. (2005). International waters strategic guidance paper. Retrieved from the Global Environment Facility website, http://sgp.undp.org/downloads/GEFSGPIWStrategicGuidancePaperFinal.pdf.

Global Environment Facility. (2006). Managing lake basins: Practical approaches for sustainable use. Retrieved from the Global Environment Facility website, http://www.worldlakes.org/uploads/draftfinalreport1.16august200 pdf.pdf.

Global Water Partnership website. Retrieved October 10, 2009, www.gwpforum.org.

Gore, C. (2008). Environment and Development in Uganda: Understanding the Global Influence on Domestic Policy in J. Diez \& O.P. Dwivedi (Eds.), Environmental management in global context: Perspectives from the South (p. 155-181). Peterborough (ON): Broadview Press.

Government of Canada. (2005). Water and Canada: Integrated water resources management, an overview of perspectives, progress, and prospects for the future at home and abroad. Ottawa (ON): Her Majesty the Queen in Right of Canada.

Graffy, E. A. (2008). Meeting the challenges of policy-relevant science: Bridging theory and practice. Public Administration Review, 68(6):1087-1100.

Great Lakes Commission. (n.d.). Great Lakes monitoring inventory and gap analysis. Retrieved March 8, 2010 from the Great Lakes Commission website, http://www.glc.org/monitoring/greatlakes/.

Grey, D. \& Sadoff. C. (2002). Beyond the river: the benefits of cooperation on international rivers. Water Policy, 4:389-403. 
Grover, B. (1998). Twenty-five years of international cooperation in water-related development assistance, 1972-1997. Water Policy, 1:29-43.

Grover, V. (August 20, 2009). Lake Twinning: Sirengthening lake management systems through twinning of African and North American lake management commissions. United Nations University - INWEH. Presentation at Stockholm World Water Week.

Hecky, R., Bootsma, H., \& Odada, E. (2006). African lake management initiatives: The global connection. Lakes \& Reservoirs: Research and Management, 11:203-213.

Hertel, S., Singer, M., \& Van Cott, D. (2009). Symposium: Fieldwork, identities, and intersectionality. Field research in developing countries: Hitting the road running. PS: Political Science and Politics, 42:305-309

Hodge, S. (2006). Knowledge innovation systems and technology diffusion strategies for ecosystem management in Africa - case study: Lake Chad basin commission. Africa Policy Journal, Fall 2:1-26.

How to break the climate impasse; If this is our last chance for a deal, let's try creative diplomacy. (2009, December 11). The Globe and Mail, Page A21.

Industry Canada. (December 3, 2002). Council of Science and Technology Advisors' report addresses challenges facing the federal science and technology. Retrieved from Industry Canada website, http:/www.ic.gc.ca/eic/site/ic1.nsf/eng/02638.html.

International Conference on Lake Victoria - LV2000: A new beginning. May 16-19, 2000. Conference recommendations. Retrieved August 8, 2009 from Lake Victoria Fisheries Organization website, http://www.lvfo.org/downloads/LVFO $\% 202000 \% 20$ Conference.pdf.

International Joint Commission. Lake Erie Task Force. Retrieved March 10, 2010 from International Joint Commission website, http:/www.ijc.org/rel/boards/letf/letfreports.html.

International Joint Commission Website. Retrieved March 10, 2010, www.ijc.org.

International Union for Conservation of Nature. Water \& nature initiative: The ecosystem approach to water management. Retrieved October 3, 2009 from the IUCN website, http://cmsdata.iucn.org/downloads/ecoman.pdf.

International Union of Conservation of Nature Website. Retrieved August 28, 2009 from the IUCN website, http://www.iucn.org/.

IRC International Water and Sanitation Centre (IRC). (1999). Integrated water resource management in water and sanitation projects: Lessons from projects in Africa, Asia and South America. Retrieved from IRC website, http://www.irc.nl/page/6302. 
Johns, C. (2008). Non-point source water pollution institutions in Ontario before and after Walkerton in M. Sproule-Jones, C. Johns, \& B.T. Heinmiller (Eds.), Canadian water politics: Conflicts and institutions (pp. 203-239). Montreal (QC): McGill-Queen's University Press.

Johns. C. (2009). Water Pollution in the Great Lakes Basin: The Global-Local Dynamic in C. Gore \& P. Stoett (Eds.), Environmental challenges and opportunities: Local-global perspectives on Canadian issues (p. 95-129). Toronto (ON): Emond Montgomery Publications Limited.

Kairu, J.K. (2001). Wetland use and impact on Lake Victoria, Kenya region. Lakes \& Reservoirs: Research and Management, 6:117-125.

Kateregga, E. \& Sterner, T.( 2009). Lake Victoria fish stocks and the effects of water hyacinth. The Journal of Environment \& Development, 18(1):62-78.

Kayombo, S. \& Jorgensen, S. (2006). Lake Victoria: Experience and lessons learned brief. Retrieved February 11, 2009 from, http://www.iwlearn.net/publications/l//lakevictoria_2005.pdf.

Keller, A. C. (2009). Science in environmental policy: The politics of objective advice. Cambridge (MA): MIT Press.

Kingdon, J. (1995). Agendas, alternatives and public policies $2^{\text {nd }}$ edition. New York: Addison, Wesley.

Kliot, N., Shmueli, D., \& Shamir, U. (2001). Institutions for management for transboundary water resources: their nature, characteristics and shortcomings. Water Policy, 3:229-255.

Klohn, W. \& Andjelic, M. Lake Victoria: A case in international cooperation. Retrieved April 10, 2008 from the Food and Agriculture Organization of the United Nations website, www.fao.org/ag/agl/aglw/webpub/lakevic/lakevic4.htm.

Lake Victoria Fisheries Organization Website. Retrieved March 3, 2009 from, www.lvfo.org.

Lake Victoria Fisheries Organization - Strategic Vision for Lake Victoria (1999-2015). Retrieved July 14, 2009 from the Lake Victoria Fisheries Organization website, http:/www.lvfo.org/downloads/LVFO Strategic_Vision.pdf.

Leach, M. \& Mearns, R. (1996). The lie of the land: Challenging received wisdom on the African environment. London (UK): James Curry Ltd.

Leech, B. (December 2002). Asking questions: Techniques for semi-structured interviews. PSOnline. Rutgers University. Retrieved from, www.apsanet.org.

Lomas, J. (Spring 2000). Connecting research and policy. Isuma, 1(1):140-144. 
Lorenz, C.M., Gilbert, A.J. \& Cofino, W. (2001a). Environmental auditing: Indicators for transboundary river management. Environmental Management, 28(1):115-129.

Lorenz, C.M., Gilbert, A.J. \& Vellinga, P. (2001b). Sustainable management of transboundary river basins: a line of reasoning. Regional Environmental Change, 2:38-53.

Ma, J., Hipel, K., De, M., \& Cai, J. (2008). Transboundary water policies: Assessment, comparison and enhancement. Water Resource Management, 22:1069-1087.

Mekong River Commission Website. Retrieved March 8, 2009 from, http://www.mrcmekong.org/about mrc.htm.

Mekong River Commission. (September 2005). News release: Water quality monitoring vital to development in the Mekong Basin. Retrieved from Mekong River Commission website, http://www.mrcmekong.org/MRC news/press05/02-nov-05.htm.

Memorandum of Understanding for Cooperation on Environment Management, East African Community Treaty. October 22, 1998. Retrieved from, http:/www.tematea.org/?q=node/5566\&PHPSESSID $=891 \mathrm{~b} 87 \mathrm{~b} 6 \mathrm{f} 8334 \mathrm{c} 5 \mathrm{db} 94 \mathrm{e} 75 \mathrm{ebc} 39 \mathrm{~d}$ ae86.

Mireri, C., Atekyereza, P., Kyessi, A. \& Mushi, N. (2007). Environmental risks of urban agriculture in the Lake Victoria drainage basin: A case of Kisumu municipality, Kenya. Habitat International, 31:375-386.

Mohd Noor, K. B. (2008). Case study: A strategic research methodology. American Journal of Applied Science, 5(11):1602-1604.

National consultative meeting between Ugandan civil society organizations and the Lake Victoria Basin Commission (LVBC) proceedings. (April 30, 2009). Retrieved from, http://hosting.digitalsphere.co.ug/ucsd/uploads/FinalpdfDraftReport $\% 20$ onLVBC $\% 20$ $\% 20 \mathrm{CSO} \% 20$ UgandaNationalConsultativeMeetingApril302009.pdf.

National Fisheries Resources Research Institute (NaFIRRI). (June 2007). The socio-economic aspects of the fishing communities of Lake Victoria, Uganda. Jinja, Uganda.

National Fisheries Resources Research Institute (NaFIRRI). (2009). The new centre of excellence for fisheries innovations in Uganda: Institute profile. Jinja, Uganda.

NeWater. New approaches to adaptive water management under uncertainty. Retrieved October 4, 2009 from NeWater website, http://www.newater.infol.

Njinu M., Kazungu, J., Ngugi, C.C., Gichuki, J. \& Muhoozi, L. (2008). An overview of the current status of Lake Victoria fishery: Opportunities, challenges and management strategies. Lakes \& Reservoirs: Research and Management, 13:1-12. 
Northey, M., Teppermen, L., Albanese, P. (2009). Making sense: A student's guide to rese'tuch and writing. Toronto (ON): Oxford University Press.

Ntiba, M., Kudoja, W. M. \& Mukasa, C.T. (2001). Management issues in the Lake Victoria watershed. Lakes \& Reservoirs: Research and Management, 6:211-216.

Odada, E. \& Olago, D. (2006). Challenges of an ecosystem approach to water monitoring and management of the African Great Lakes. Aquatic Ecosystem Ilealth \& Management, 9(4):433-446.

Ontario Ministry of the Environment and Energy. (1993). Water Management on a watershed basis: Implementing an ecosystem approach. Toronto (ON): Queen's Printer for Ontario.

Orach-Meza, F.L. \& Okurut, T. (January 2005). Background to the preparation of the second phase of the Lake Victoria environmental management project (LVEMP'-II).

Presentation at The Regional Stakeholders' Workshop on Launching the Preparation of the LVEMP Phase Two.

Pringle, R. (2005). The Nile perch in Lake Victoria: Local responses and adaptations. Africa, $75: 4,510-538$.

Regional status report on Lake Victoria: Frame surveys for 2000, 2002 and 2004. Lake Victoria environmental management project (LVEMP) and Lake Victoria Fisheries Organization (LVFO). Retrieved May 12, 2009 from the Lake Victoria Fisheries Organization website, http://www.lvfo.org/downloads/Regional\%20frame $\% 20$ survey $\% 202000-2004$.pdf.

Republic of Kenya, Ministry of the Environment and Natural Resources. (2008). Lake Victoria environmental management project phase II (LVEMP - II) preparation: Final report.

Retrieved February 14, 2009 from,

http://wwwwds.worldbank.org/external/default/WDSContentServer/WDSP/II3/200)8/(03/1 $1 / 000333038$ 20080311055406/Rendered/PDF/E18320VOL(0400P100406,00130) 32144 3B.pdf.

Reynolds, C.S. (1993). The ecosystems approach to water management. The main features of the ecosystems concept. Journal of Aquatic Ecosystem Health, 2:3-8.

Rubin, H. \& Rubin, S. (1995). Qualitative interviewing: The art of hearing data. Thousand Oaks (CA): Sage.

Sadoff, C., Whittington, D. \& Grey, D. (2002). Africa's international rivers: An economic perspective. Washington (D.C.): World Bank Publications.

Salman M. \& Salman, A. (2009). The World Bank policy for projects on internutional waterways: An historical and legal analysis. Washington (D.C.): World Bank Publications. 
Schaefer, K. \& Bielak, A. (2006). Linking water science to policy: Results from a series of national workshops on water. Environmental Monitoring and Assessment, 113:431-442.

Science 2008: A question of democracy. March/April 2008. Seed Magazine, p. 22.

Shenhav, Y. \& Kamens, D. (1991). The 'costs' of institutional isomorphism: Science in nonwestern countries. Social Studies of Science, 21:527-45.

Shields, P. \& Tajalli, H. (2006). Intermediate theory: The missing link to successful student scholarship. Texas State University, Department of Political Science. Retrieved from Texas State University website, http://ecommons.txstate.edu/polsfacp/39.

Sproule-Jones, M. (2002). Restoration of the Great Lakes: Promises, practices, performances. Vancouver (B.C.): UBC Press.

Sturges, J. \& Hanrahan, K. (2004). Comparing telephone and face-to-face qualitative interviewing: a research note. Qualitative Research, 4(1):107-118.

The Convention for the Sustainable Development of Lake Tanganyika. (June 12, 2003). Retrieved from, http://ocid.nacse.org/tfdd/tfdddocs/427ENG.pdf.

Timmerman, J. \& Langaas, S. (2005). Water information: what is it good for? The use of information in transboundary water management. Regional Environmental Change, 5:177-187.

United Nations Environment Programme (UNEP). (n.d.) Lake Victoria Basin: Environment outlook. Retrieved May 1, 2010 from, http://www.unep.org/dewa/africa/docs/en//vicbasin brochure.pdf.

United Nations University, Institute for Water, Environment and Health. (September 24-26, 2008). Minutes of Lake Twinning Workshop on science and policy linkages. Entebbe. Uganda. Retrieved from the United Nations University website, http://www.inweh.unu.edu/River/Entebbe $\% 20$ Workshop $\% 20$ minutes.pdf.

United Nations (UN)-Water. (May 2008). Status report on integrated water resources management and water efficiency plans prepared for the 16th session of the Commission on Sustainable Development. Retrieved January 26, 2009 from United Nations website, http://www.unwater.org/downloads/UNW Status_Report IWRM.pdf.

United States Environmental Protection Agency. Effects of acid rain - surface waters and aquatic animals. Retrieved March 8, 2010 from EPA website, http://www.epa.gov/acidrain/effects/surface water.html.

Varis, O., Tortajada, C. \& Biswas, A. K. (2008). Management of transboundary rivers and lakes. Netherlands: Springer. 
Verschuren, D., Johnson, T.C., Kling, H.J., Edgington, D.N., Leavitt, P.R., Brown, E.T., Talbot, M.R., \& Hecky, R.E. (2001). History and timing of human impact on Lake Victoria, East Africa. The Royal Society, 269:289-294.

White, G. (1998), Reflections on the 50-year international search for integrated water management. Water Policy, 1: 21-27.

World Bank. Region: Sub-saharan Africa. What is indigenous knowledge? Retrieved March 13, 2010 from World Bank website, http://www.worldbank.org/afr/ik/basic.htm.

Wright, R. (2004). A short history of progress. CBC Massey Lectures. Toronto (ON): House of Anansi Press.

Wynn, B. \& Mayer, S. (1993). How science fails the environment: If politicians don't like uncertainties and scientists often conceal them, how can we make policies that deal with the vagaries of the world around us? New Scientist. Retrieved September 14, 2008 from, http://www.newscientist.com/article/mg13818764.000-how-science-fails-theenvironment-if-politicians-dont-likeuncertainties-and-scientists-often-conceal-them-howcan-we-make-policiesthat-deal-with-the-vagaries-of-the-world-around-us.html. 


\section{Thesis Interview Guide:}

\section{Question Themes*:}

(1) Please identify self and role and functions in position, and role and function of your organization.

(2) Reflect on experience with policymaking generally, the science-policy interface, i.e. inclusion of scientific information in the policy process, and identify gaps/challenges, if any.

(3) Discuss participation in the development of transboundary water management policies and design of management regimes, if applicable.

(4) Reflect on experience in transboundary water management and identify successes, challenges, mechanisms for stakeholder participation, role of international donors and lending institutions (if applicable) and how these will influence the future of transboundary water management.

(5) Discuss the inclusion of (or lack thereof) science in transboundary water management regimes and policies and the extent that science influences the success and implementation of management policies and practices.

(6) Discuss how science is researched and incorporated into management policies and plans to change or alter the way that science is integrated in the future.

(7) Discuss how the knowledge of non-government interests (community groups, nongovernment organizations, general public) is integrated into policy decisions or management regimes.

'Question themes were modified and tailored to the candidates' expertise, organizational affiliation and involvement in environmental policy development. 
Dear $<$ Candidate $>$,

My name is Christina Cheng and I am a Master's candidate at Ryerson University in Toronto, Ontario, Canada. My thesis examines the science-policy interface in transboundary water management regimes and the influence of science on the success of transboundary water policies, with a case study on East Africa's Lake Victoria.

Through my research, I understand you have been and/or are currently involved with the development of transboundary water management policy in the <name of water body $>$ and $<$ include other relevant information $>$. It is your experience with these activities that I am contacting you to request an in-person or telephone interview to gather intelligence for my thesis.

Your participation in the interview is strictly voluntary and will last for approximately one hour. A list of interview questions/themes is attached to provide you with an indication of the types of questions that will be asked should you agree to participate. A consent agreement will be sent to you and will need to be signed in advance of our interview.

I can be reached via email at Christina.Cheng(aryerson.ca or at (416) xxx-xxxx to schedule a mutually convenient time and location for the interview. If possible, I would like to schedule the interview within one month. Should you have any immediate questions or require clarification of the request, please do not hesitate to contact me.

Thank you for your time and consideration.

Christina 


\section{Study Title: Examining the Science-Policy Interface in Transboundary Water Management Regimes}

You are being asked to participate in a research study. Before you give your consent to be a volunteer, it is important that you read the following information and ask as many questions as necessary to be sure you understand what you will be asked.

Investigator: Christina Cheng, B.Sc. (Hons)

\section{Purpose of the Study:}

There are over 260 water bodies throughout the world that border two or more countries. These water bodies represent significant ecological, economic and social resources for countries and the world. Thus, the need for effective, integrated and collaborative water management regimes is pressing.

The purpose of this study is to understand the challenge of integrating scientific information and knowledge into transboundary water management. Specifically, the study will identify ways in which science is incorporated into multilateral water management regimes and compare how these systems are similar or different in both developed and developing countries, with a focus on East Africa's Lake Victoria. This research will help provide knowledge about how different national and international contexts influence the integration of science into management and policy.

\section{Confidentiality:}

Any information conveyed during interviews will be kept strictly confidential. Your identity will also be kept confidential, meaning that your knowledge and/or your participation will not be shared or disclosed. If I wish to share publicly any information you provide in our interview, I will seek your permission prior to using this information. Draft excerpts will be shared with you prior to inclusion in the final thesis document to ensure information accuracy and to reconfirm your permission.

The data collected for this study will be used for academic purposes only. Records will be kept strictly confidential and only the investigator will have access to interview data. Data will be stored securely at the researcher's office for a period up to five years subsequent to the completion of the thesis and then will be destroyed. Confidentiality will be maintained to the extent allowed by law.

\section{$\underline{\text { Risks }}$}

The risk of participating in this interview is the potential disclosure of sensitive information you share that may result in adverse professional and economic consequences, such as loss of employment. The researcher will take necessary precautions to ensure the information provided in this interview is kept strictly confidential. 


\section{Benefits}

The knowledge gained from this interview will be valuable for understanding the interface between scientific knowledge, policymaking and transboundary water management. It will contribute to global and domestic research and knowledge about transboundary water management and opportunities and challenges of improving management practices and policy solutions. The observations and insights from the study will be shared with all interview participants when the thesis is completed.

Incentives to Participate: Participants will not be paid to participate in this study.

Voluntary Nature of Participation: Participation in this study is voluntary. Your choice of whether or not to participate will not influence your future relations with Ryerson University. If you decide to participate, you are free to withdraw your consent and to stop your participation at any time. At any particular point in the study, you may refuse to answer any particular question or stop participation altogether. Should you wish, your responses to interview questions can be erased and destroyed.

Questions about the Study: If you have any questions about the research now, please ask. If you have questions later about the research, you may contact me or my academic advisor directly:

Christina Cheng, Hon.BSc.

Graduate Program, Environmental Applied Science and Management

Telephone Number: (416) xxx-xxxx

Christina.Cheng@ryerson.ca

Dr. Christopher Gore, Assistant Professor

Department of Politics and Public Administration

Associate Graduate Program in Environmental Applied Science and Management

Telephone Number: (416) 979-5000 ext. xxxx

Chris.Gore@ryerson.ca

If you have questions regarding your rights as a human subject and participant in this study, you may contact the Ryerson University Research Ethics Board for information.

Research Ethics Board

c/o Office of the Vice President, Research and Innovation

Ryerson University

350 Victoria Street

Toronto, ON M5B 2K3

416.979 .5000 ext. 7112

The undersigned hereby acknowledges his/her review and understanding of the consent agreement and consents to participate in the study. 
Name of Participant (please print)

Date

Permission to be audio-taped

Signature of Participant

Signature of Participant 
